\title{
ROADMAP TO THE SRS COMPUTING ARCHITECTURE
}

by

Johnson, A.

Westinghouse Savannah River Company

Savannah River Site

Aiken, South Carolina 29808

DOE Contract No. DE-AC09-89SR18035

This paper was prepared in connection with work done under the above contract number with the U.S.

Department of Energy. By acceptance of this paper, the publisher and/or recipient acknowledges the U.S. Government's right to retain a nonexclusive, royalty-free license in and to any copyright covering this paper, along with the right to reproduce and to authorize others to reproduce all or part of the copyrighted paper. 


\section{DISCLAIMER}

This report was prepared as an account of work sponsored by an agency of the United States Government. Neither the United States Government nor any agency thereof, nor any of their employees, make any warranty, express or implied, or assumes any legal liability or responsibility for the accuracy, completeness, or usefulness of any information, apparatus, product, or process disclosed, or represents that its use would not infringe privately owned rights. Reference herein to any specific commercial product, process, or service by trade name, trademark, manufacturer, or otherwise does not necessarily constitute or imply its endorsement, recommendation, or favoring by the United States Government or any agency thereof. The views and opinions of authors expressed herein do not necessarily state or reflect those of the United States Government or any agency thereof. 


\section{DISCLAIMER}

Portions of this document may be illegible in electronic image products. Images are produced from the best available original document. 


\section{Roadmap to the SRS Computing Architecture (U)}

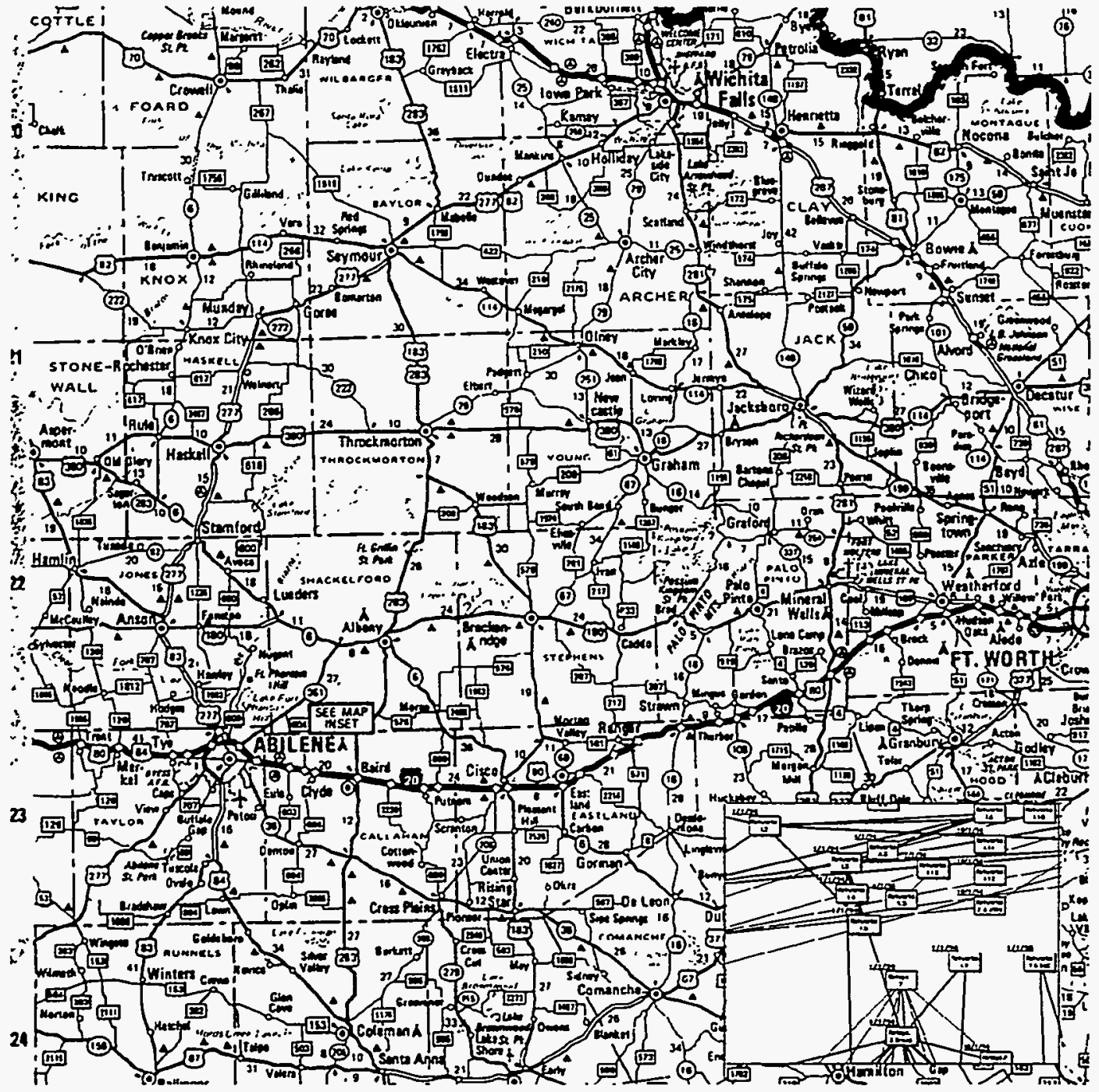

\section{IRM}

(1)

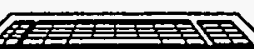

Information

Resource

Management
Westinghouse Savannah River Company

Savannah River Site

Aiken, SC 29808

Prepared for the U. S. Department of Energy Under Contract No. DE-AC09-89SR18035 


\section{Roadmap to the}

SRS Computing Architecture (U).

Publication Date: July 5, 1994

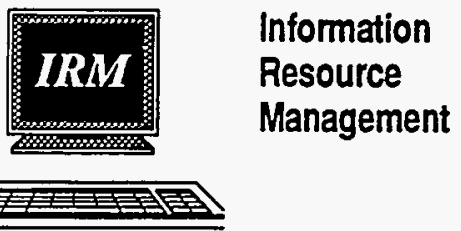

Westinghouse Savannah River Company

Savannah River Site

Aiken, SC 29808

Prepared for the U. S. Department of Energy Under Contract No. DE-ACO9-89SR18035

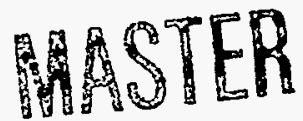

DISTRIBUTION OF THIS DOCUMENT IS UNLIMITED $f 7 \delta$ 


\section{Table of Contents}

II. Advanced Networks Roadmap............................................................................... 3

Introduction and Destination...............................................................................................3

Starting Point ...................................................................................................................................

Networks Infrastructure Milestones .............................................................................................10

Networks.I.1 Install the Replacement Telephone System .............................................11

Networks.I.2 Complete Inter-area Cabling .........................................................................12

Networks.I.3 Complete Site Universal Wiring ....................................................................13

Networks.I.4 Complete Building LANs.............................................................................14

Networks.I.5 Install Ethernet/FDDI Network Trunks .......................................................15

Networks.I.6 Install Switched Ethernet Hubs ...................................................................16

Networks.I.7 Install ATM Network Trunks .....................................................................18

Networks.I.8 Install ATM Workgroup Networks .................................................................20

Networks.I.9 Complete Network Management System ....................................................21

Networks.I.10 Initiate Network Authentication Service.......................................................22

Networks.I.11 Install Wireless LANs as Extension to SRSnet...........................................23

Networks.I.12 Complete Analysis of Network, Telephone, and Dedicated Fiber Usage Requirements .....................................................................................24

Networks.I.13 Provide Access Between SRSnet and Offsite Locations.............................25

Networks Administrative Milestones ....................................................................................226

Networks.A.1 Set Site Direction, Funding, and Priority ......................................................27

Networks.A.2 Relocate Most Network Users Onsite ............................................................28

Networks.A.3 Resolve Security and Spectrum Management Issues for Wireless

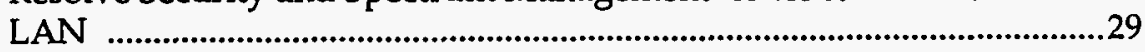

Networks.A.4 Obtain Computer Security Endorsement of Network-based Authentication ................................................................................................30

Networks.A.5 Resolve Security and Network Use Issues for Offsite Access to and

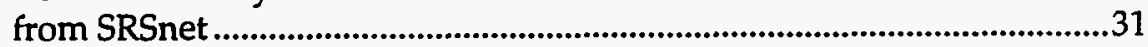

Networks Technology Milestones ..........................................................................................32

Networks.T.1 Implement Cost-effective Switched Ethernet Hubs .....................................33

Networks.T.2 Complete ATM Standards...............................................................................34

Networks.T.3 Complete ATM Interfaces for Routers/Hubs..............................................35

Networks.T.4 Complete ATM Interfaces for Workstations/ Host Computers ................36

Networks.T.5 Make DCE Security Services Available in Key Environments..................37

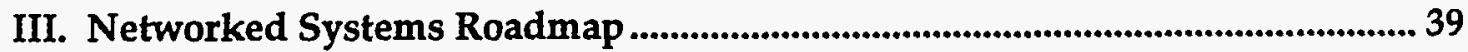

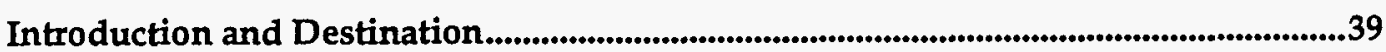

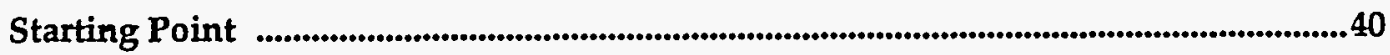

Network Systems Infrastructure Milestones.....................................................................42

Netsys.I.1 Install Robust Client Workstations ...............................................................43

Netsys.I.2 Upgrade Workstations to Support Netsys Requirements ........................44 
Netsys.I.3 Ensure Broad Availability of Networked Workstations ......................... 45

Netsys.I.4 Migrate to New NOS.........................................................................4 46

Netsys.I.5 Initiate NOS Global Directory ...........................................................4 47

Netsys.I.6 Initiate Network Authentication Service ................................................ 48

Netsys.I.7 Complete Installation of Territorial File Servers......................................49

Netsys.I.8 Manage IP Addresses..........................................................................5 50

Netsys.I.9 Implement Robust File/Print/Application Services...............................51

Netsys.I.10 Implement Automatic Software Distribution......................................... 52

Netsys.I.11 Connect Office Peripherals Directly to Network .....................................53

Netsys.I.12 Implement Reliable Performance Monitoring ......................................... 54

Network Systems Administrative Milestones................................................................ 55

Netsys.A.1 Set Site Direction, Funding, and Priority .................................................56

Netsys.A.2 Define Security Principles .................................................................... 57

Netsys.A.3 Establish NOS Operations Group ....................................................... 58

Network Systems Cultural Milestones..........................................................................59

Netsys.C.1 Implement Standards ....................................................................6 60

Netsys.C.2 Share Equipment/Peripherals ............................................................6 61

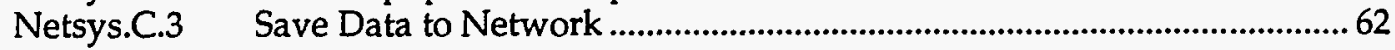

Network Systems Process Milestones ............................................................................6 63

Netsys.P.1 Implement Single User Login to Network...............................................6 64

Netsys.P.2 Implement Distributed Workgroup Management.................................... 65

Netsys.P.3 Implement Software License Management ..........................................66

Netsys.P.4 Implement Disaster Recovery Procedure .............................................67

Netsys.P.5 Implement Infrastructure Enhancement Process ....................................6 68

Network Systems Technology Milestones.......................................................................69

Netsys.T.1 Deliver NOS over TCP/IP .....................................................................70

Netsys.T.2 Implement Transparent Print Services on All Platforms..........................71

Network Systems Miscellaneous Milestones ............................................................72

Netsys.M.1 Implement PC Utilization Plan ............................................................ 73

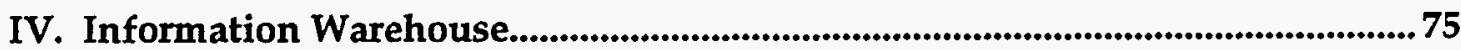

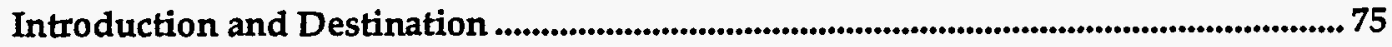

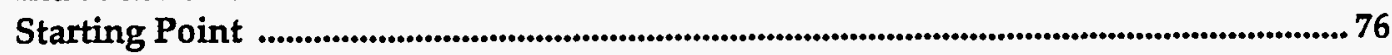

Information Warehouse Infrastructure Milestones ................................................. 77

IW.I.1 Establish a Communications Network Capability Supporting the Global and Local Warehouses ....................................................................... 78

IW.I.2 Establish Client SQL-Network ...............................................................79

IW.I.3 Establish/Populate IW Component of Data Repository ..........................8 80

IW.I.4 Identify and Procure IW Support Tools and Hardware..........................82

IW.I.5 Identify and Procure Archival Management Tools and Hardware........83

Information Warehouse Administrative Milestones ........................................................8 84

IW.A.1 Establish IW Implementation Team .....................................................8 85

IW.A.2 Establish Long-term Support Organization ...........................................86

IW.A.3 Establish IW Polices and Procedures ......................................................... 8 87

IW.A.4 Replace Redundant Operational Reports, Screens, and Data Feeds ...... 88

Information Warehouse Cultural Milestones .................................................................89

IW.C.1- $\quad$ Establish Training for Application-development Community ............... 90

IW.C.2 Establish Training for End-User Community .......................................92 
Information Warehouse Process Milestones.

IW.P.1

Identify and Prioritize Business Requirements for Global and

Local Warehouses.

IW.P.2

Define the IW Model

IW.P.3

Implement the Global Warehouse.

IW.P.4

Expand Global Warehouse.

IW.P.5

Establish Local or Remote IWs

IW.P.6

Establish Archival IW

V. Client/Server Roadmap

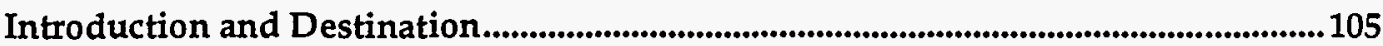

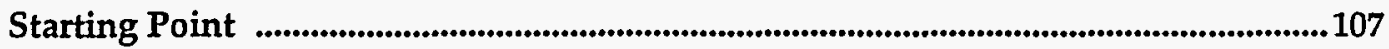

Client/Server Infrastructure Milestones .............................................................................109

C/S.I.1 Install Robust Development Workstations .................................................110

C/S.I.2 Implement Locally Distributed, Centrally Managed

C/S.I.3 Implement Single Network Transport Protocol for Client/Server Communication ..................................................................................112

Client/Server Administrative Milestones ..................................................................113

C/S.A.1 Develop Graphical User Interface Guidelines .............................................114

C/S.A.2 Develop Naming Conventions..............................................................115

C/S.A.3 Enable Sitewide Data Access ..........................................................................116

C/S.A.4 Encourage Move Toward Client/Server Development..........................117

C/S.A.5 Migrate Selected Host-Based Legacy Systems to Client/Server.............118

Client/Server Culture Milestones .................................................................................................120

C/S.C.1 Establish Small Development Team Methodology ...................................121

C/S.C.2 Establish Policies Favoring Iterative Software Development.................. 122

Client/Server Process Milestones.............................................................................................123

C/S.P.1 Implement Standard Development Methodology and Tools Across

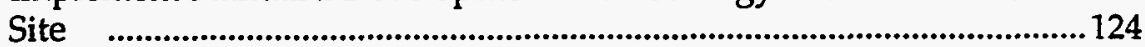

C/S.P.2 Integrate Software Tools, Development Methodology, Project, and Configuration Management Products .......................................................125

Client/Server Technology Milestones .....................................................................................127

C/S.T.1 Implement Standard RDBMS Accessibility ..............................................128

C/S.T.2 Implement Graphical Report Generators and Query Tools .....................129

C/S.T.3 Implement Nonrelational Database Access Through SQL Gateway.... 130

C/S.T.4 Implement Declarative Referential Integrity, Triggers, and Stored Procedures Supported by RDBMS ................................................................131

C/S.T.5 Implement Monitoring Tools ............................................................... 132

C/S.T.6 Implement Cross-platform Two-phase Commit Regardless of Vendor ............................................................................................. 133

VI. Multimedia Roadmap ...........................................................................................135

Introduction and Destination.....................................................................................................135

Starting Point .............................................................................................................................. 136

Multimedia Infrastructure Milestones............................................................................138

MMedia.I.1 Develop Multimedia Principles .....................................................................1139

MMedia.I.2 Adopt Cross-platform Fonts and Image Formats ......................................140

MMedia.I.3 Establish Navigable Compound Document Format ................................. 141 
MMedia.I.4 Enable Routine Capture and Playback of Cross-platform Audio 142

MMedia.I.5 Implement Storage, Multimedia Servers, CD-ROM, and Improved Monitors ....

MMedia.I.6

MMedia.I.7

Enable Playback Motion Video at Desktop

144

MMedia.I.8

Implement Workgroup Video-capture Stations.

145

Multimedia Administrative Milestones

146

Approve Use of Multimedia Data Types

MMedia.A.2 Formulate Policy for Authentication of Nonprintable Data Types ...... 149

MMedia.A.3 Determine Business Case for Desktop Video Capture ............................. 150

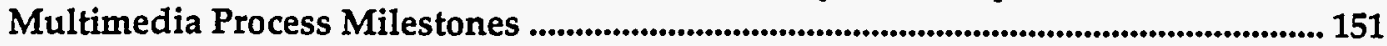

MMedia.P.1 Manage Stored Digital Video Clips.................................................... 152

MMedia.P.2 Implement Routine Use of Multimedia Data in Office Documents ..... 153

Multimedia Technology Milestones............................................................................ 154

MMedia.T.1 Pilot Multimedia Enablement of Desktop Products ................................. 155

MMedia.T.2 Pilot Navigable Compound Documents.................................................. 156

MMedia.T.3 Pilot Speech and Handwriting Recognition ......................................... 157

MMedia.T.4 Pilot Applicability of Object Databases to Multimedia .......................... 158

MMedia.T.5 Pilot Desktop Videoconferencing ................................................................... 159

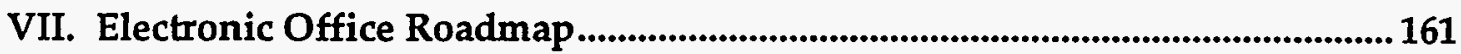

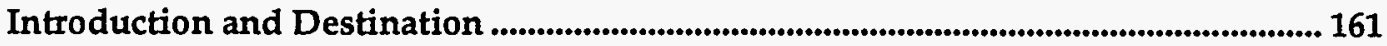

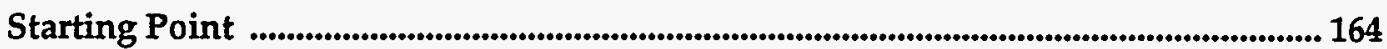

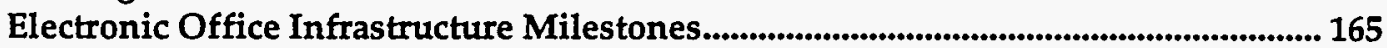

Office.I.1 Implement FAX Input/Output....................................................... 166

Office.I.2 Install Online Scanners ........................................................................ 167

Office.I.3 Install Ubiquitous OCR .................................................................... 168

Office.I.4 Install Client/Server E-mail................................................................. 169

Office.I.5 Implement Mail-enabled Applications ................................................... 171

Office.I.6 Implement Electronic Forms Routing and Authorization....................... 172

Office.I.7 Implement Viewers/Translators ................................................................. 173

Office.I.8 Integrate Voice-mail and E-mail Systems .................................................... 175

Electronic Office Administrative Milestones ..................................................................... 177

Office.A.1 Implement Security Policy and Procedures............................................... 178

Office.A.2 Scan in Historical and Reference Data................................................... 179

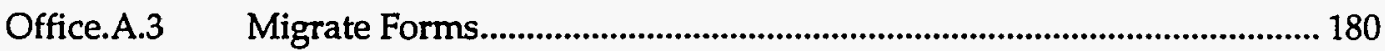

Office.A.4 Implement Online Librarian and Document Repository.......................... 181

Office.A.5 Recognize Electronic Signatures as Binding.......................................... 182

Office.A.6 Implement the Paperless Office.................................................................. 183

Electronic Office Process Milestones...................................................................................... 184

Office.P.1 Implement Document Management System ........................................... 185

Office.P.2 Electronic Data Interchange..................................................................... 186

Electronic Office Technology Milestones .............................................................................. 187

Office.T.1 Implement Voice Processing.................................................................... 188

VIII. Object-oriented Technology ................................................................. 189

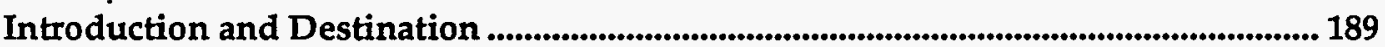

Starting Point .......................................................................................................................... 190 


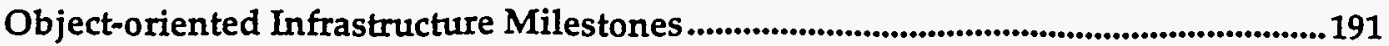

Object.I.1 Establish Site Object Framework Team (SOFT) ......................................192

Object.I.2 Adopt Site Object Messaging Standard...................................................193

Object.I.3 Establish Object Framework ….................................................................. 194

Object.I.4 Determine and Irnplement the Necessary Changes to WISDOM to Support an Object-oriented Environment..................................................195

Object.I.5 Re-engineer Existing Core Applications as Object Applications ...........196

Object-oriented Administrative Milestones....................................................................197

Object.A.1 Direct that Future Site Applications Should Be Object-oriented............ 198

Object.A.2 Procure Object-oriented Technology ..........................................................199

Object.A.3 Develop Object Migration Plans for Existing Systems............................200

Object-oriented Cultural Milestones ..............................................................................2202

Object.C.1 Implement Matrix Project Team Approach ….........................................203

Object.C.2 Develop Site Expertise In Object-oriented Technology ............................204

Object.C.3 Establish Reward System for Code Reuse ....................................................205

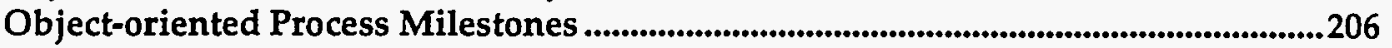

Object.P.1 Establish Object-oriented Documentation Requirements.......................207

Object.P.2 Establish Object-oriented Development Methodologies ..........................209

Object.P.3 Implement Online, Centrally Managed, Distributed Access for Site Object Framework Developer Documentation.........................................210

Object-oriented Technology Milestones.................................................................................211

Object.T.1 Survey Industry Experience in Migrating Legacy Systems to an Object-oriented Environment.........................................................................212

Object.T.2 Demonstrate Relational Database Access from Object-oriented Systems ...........................................................................................................2213

Object.T.3 Evaluation of Operating Systems (Object-oriented).................................214

Object.T.4 Evaluation of Object-oriented Development Tools.....................................215

Object.T.5 Explore Distributed Object Server Technology .............................................216

IX. Bibliography

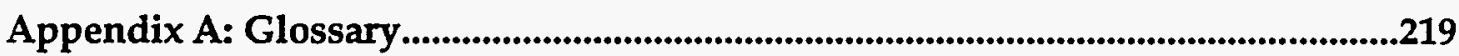

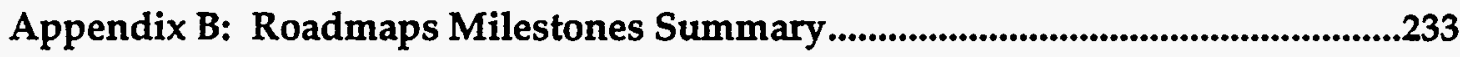




\section{Introduction}

This document outlines the major steps that must be taken by the Savannah River Site (SRS) to migrate the SRS information technology (IT) environment to the new architecture described in the Savannah River Site Computing Architecture. This document proposes an IT environment that is

... standards-based, data-driven, and workstation-oriented, with larger systems being utilized for the delivery of needed information to users in a client-server relationship.

Achieving this vision will require many substantial changes in the computing applications, systems, and supporting infrastructure at the site. This document consists of a set of roadmaps which provide explanations of the necessary changes for IT at the site and describes the milestones that must be completed to finish the migration.

The roadmaps provided here group the necessary changes by the following technologies:

Advanced Networks (Networks) - The upgrade of the site data network, SRSnet, to one that is robust and reliable enough to support the architecture's requirements.

Networked Systems (Netsys) - Steps to upgrade the site's personal computers (workstations) and supporting networked services infrastructure to the capabilities required by future site systems and applications.

Information Warehouse (IW)-Steps to establish a managed information environment where data specific to the management of an enterprise is extracted from the data collections or operational environment and migrated to an integrated reporting environment that is specifically tuned to query and end- user access.

Client/Server (C/S)-Steps to develop applications based on the client/server model. An application is said to be a C/S application when one computer, usually on a desktop, performs some of the functions of an application and requests services of other computers across a computer network to provide the remainder of the application function.

Multimedia (MMedia) - Steps to develop applications based on multimedia data types. Multimedia is a collection of technologies that combines the formats of text, voice, video, audio, image, and animation for delivery to workstations, specialized platforms, or existing standard audio visual equipment.

Electronic Office (Office)-Steps to develop an environment in which common office functions, which depend on manual and paper-oriented processes today, are performed using computers and communications networks.

Object Oriented Technology (Object)—Steps to convert the site application acquisition, development, and support environment to one based on the concepts of object-oriented programming (OOP). 
For each technology area, the document outlines the goals envisioned for the technology (that is, the Destination), the current state of the site with respect to the technology (Starting Point), and a set of milestones grouped as follows:

1. Infrastructure Milestones-Milestones pertaining to the site computing infrastructure which must be resolved to adopt the technology.

2. Administrative Milestones-Milestones pertaining to organizational and management issues which must be resolved to adopt the technology.

3. Cultural Milestones-Milestones that pertain to the site culture which must be resolved to adopt the technology.

4. Process Milestones-Milestones that pertain to work flow that must be resolved to adopt the technology.

5. Technology Milestones-Milestones that pertain to the technology itself that must be resolved to adopt the technology.

6. Miscellaneous Milestones-Additional milestones not covered by any other issue area that must be resolved to adopt the technology.

Note that neither the roadmaps nor the milestones are necessarily sequential in nature. It is not only possible but desirable to pursue as many of them in parallel as possible. It is equally important to realize that no document of this type can ever be complete or up-to-date. The destination is, by definition, a moving target and can never be completely defined. Similarly, the starting point is also moving as the site itself adopts new technologies and capabilities, and drops or modifies old ones. Moreover, the technology itself is constantly evolving a new set of opportunities for migration. In view of this situation, a document of this kind represents a snapshot of a point in time. 


\section{Advanced Networks Roadmap}

\section{Introduction and Destination}

Ideally, a computer network should provide transparent and unrestricted data transport services to its attached computer systems, the applications that run on these systems, and the personnel that use the applications. That is, if a user or application requires access to data or computational services that are available on one part of the network, the network has the capability to provide access to this data or service without (significant) delay and without placing undue navigation or access requirements on the user. This capability is desired regardless of the distances or the amount of data involved, and (again ideally) the user of such a facility should not be required to be aware of the network's existence and certainly not aware of the details of the network topology.

Figure 1 below illustrates this facility. The user at network location $A$ uses an application that executes at location $B$ and accesses data from location $C$. The network is drawn as an oval, but in reality, the network topology will be complex and relatively arbitrary.

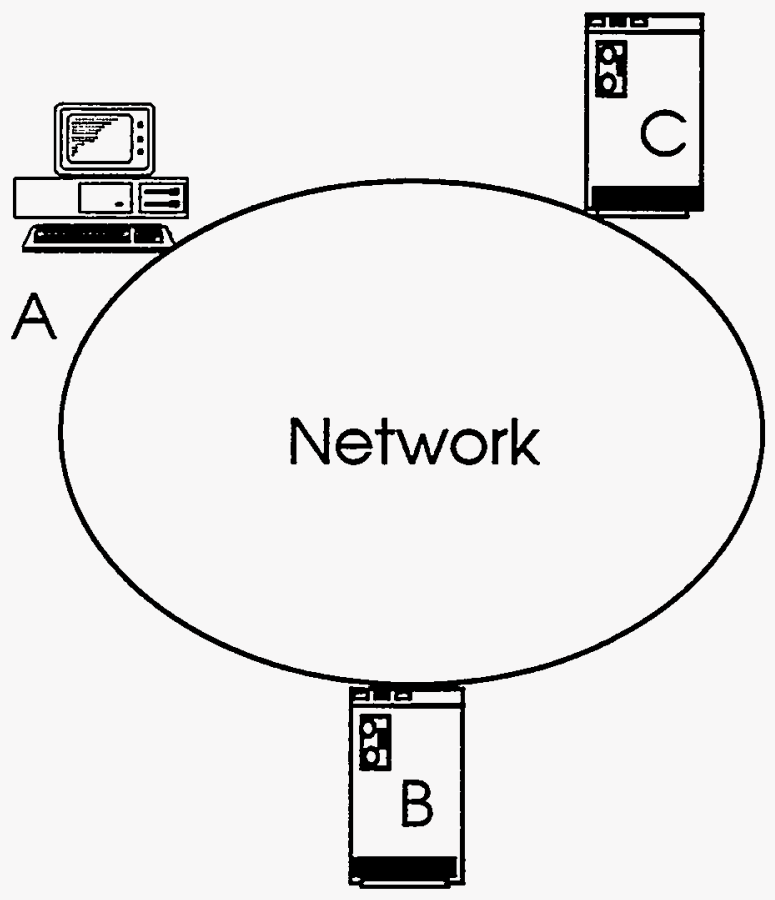

Figure 1: Typical network of today.

If the network is to be transparent in this case, then the network must possess the following attributes: .

The network must be reliable.

If the network is not reliable, then it is certainly not transparent to the user. Reliability is a relative concept and also involves tradeoffs with cost of implementation and flexibility. Network reliability is created either by improving the reliability of the individual components of the network or by 
building in redundancy in the manner in which the individual components (communications lines and the processors used to direct traffic over the communications lines) are implemented.

The network must be robust.

A robust network has the capacity to serve all its users without failure and without significant (that is, users are aware of) delay. For example, if an application requires a million characters of data to be transferred between locations and the slowest connection link in the path between the two locations supports only 1,000 characters per second, then in the best case, the user of the application will be aware of 1,000 seconds of delay. If other network systems are also attempting to utilize this same link at the same time, then the delay will be much longer.

Robustness is, of course, also a relative term. What we called robust yesterday is painfully slow today. Nevertheless, a robust network must have the capability to support all users and application requirements without introducing significant delay.

There must be network-wide user authentication.

The user is required to authenticate him- or herself only once (that is, supply a user identification and confidential password) at the network level. The user must not be required to authenticate at every node on the network which supplies the user data or services. With network-wide authentication, the user authenticates to a security authentication server once, and this device communicates authentication information when required by service nodes.

The network of the future is expected to be based on the following key technologies:

\section{Switched Ethernet Hubs}

Traditional Ethernet hubs provide Ethernet local area network (LAN) segments based on unshielded twisted pairs (UTP) of wire (that is, telephone wire) connected from the users' workstations to the hub in a star configuration. Every user connected to the hub shared a single Ethernet. With switched Ethernet hubs (SEH), each user is given a dedicated Ethernet port at 10 million bits per second (mbps). Traffic from this user is directed to a single destination port on the hub. In this way, high bandwidth communications between two hub-connected computers do not impact the other nodes on the hub. Indeed, be many simultaneous file transfers can take place within the hub with the aggregate data transfer rate far in excess of Ethernet's 10 mbps.

As described, SEH provide an ideal workgroup network environment for peer-to-peer applications. However, since many network applications are not peer-to-peer but rather many-to-one (for example client/server applications, file servers, and communications servers such as network routers), SEH needs to provide the capability to link the hub users with network servers at higher speeds. This capability prevents a bottle neck at the server. Fiber Distributed Data Interface (FDDI) and Asynchronous Transfer Mode (ATM) technologies will be used to join the SEH to these servers. 
- Fiber Distributed Data Interface

FDDI is a 100 mbps LAN technology based on fiber optic technology. It is now well accepted as a standard LAN. FDDI adapters are available for all common workstations, host computers, and communications servers. FDDI technology is suitable for both work group LANs and network trunks. Because it is a shared medium type of LAN, data rates a vailable to individual users will always be less than $100 \mathrm{mbps}$ (and often much less). For this reason, FDDI it is expected to be phased out in favor of ATM (described below) over time. However, FDDI is now a mature standard that promises near-term expansion over Ethernet data capabilities, while ATM is not yet mature.

\section{Asynchronous Transfer Mode LANs and WANs}

ATM, also called cell relay, is an emerging communications technology that promises to revolutionize the way data networks are built. ATM is based on the ability to break all communications (voice, video, data) into small (53 byte) packets called cells. An ATM network consists of relay nodes that can switch incoming cells to the appropriate outgoing communications line. These devices are characterized by high capacity (millions of cells per second) and low latency (very little delay in a cell going through the device). Combined with fiber optics communications technology that permits high data rates at long distances, this technology blurs the distinction between local area networks and wide area networks. Indeed, with a fully implemented ATM network, data traffic could theoretically flow between any two SRS locations at the same high speeds, regardless of locations or distances involved. ATM devices used to support high performance local area networks are called ATM hubs. ATM switches are used to build wide area ATM networks. ATM will be implemented at various data rates from $55 \mathrm{mbps}$ to over a gigabit (a billion bits) per second.

\section{Network Management}

There are now standards for network device management (Simple Network Management Program, Common Management Information Protocol) that are the key components required to build an effective network management system (NMS). In such a system, each network component (workstations, servers, hosts, routers, bridges, hubs, etc.) would report routinely and on demand to a network management station its current operational status and would also allow configuration and operational changes to be set in the network devices from a single station.

\section{Network Authentication}

A network supporting network-wide user authentication requires a common protocol for user authentication that is supported by the operating system and appropriate applications software on all platforms (user workstations, host computers, and servers) attached to the network. For example, the personal computer terminal emulation and data access software must support a common network authentication protocol, as must the security portions of the operating systems for the IBM hosts, the VAX hosts, the UNDX hosts (including the Cray), and the network file and print servers. Fortunately, there is such a protocol that appears to be gaining acceptance across these platforms, the Distributed Computing Environment (DCE) Security Services protocol. This protocol, an outgrowth of the MIT 
Kerberos protocol, provides for network security servers. Users authenticate themselves at the security servers via the exchange of user ID and password. If a user needs access to a network service or data, the security server provides an authenticated permission to the host system.

\section{Wireless LAN Extensions}

The capability to extend the LAN with wireless technology is needed to provide cost effective network connections in those situations where rewiring a facility is cost prohibitive or restricted for other reasons. Wireless technology also supports the concept of mobile computing, that is, the ability to utilize a workstation from anywhere on the site, in the local area, or even in the world to connect to services and data sources provided by SRSnet. The technology is available now; implementations will depend on the ability to resolve security and frequency management issues.

Figure 2 shows how a network utilizing these technologies could look. Note that the slowest link between any two personal computers in this figure is a dedicated $10 \mathrm{mbps}$.

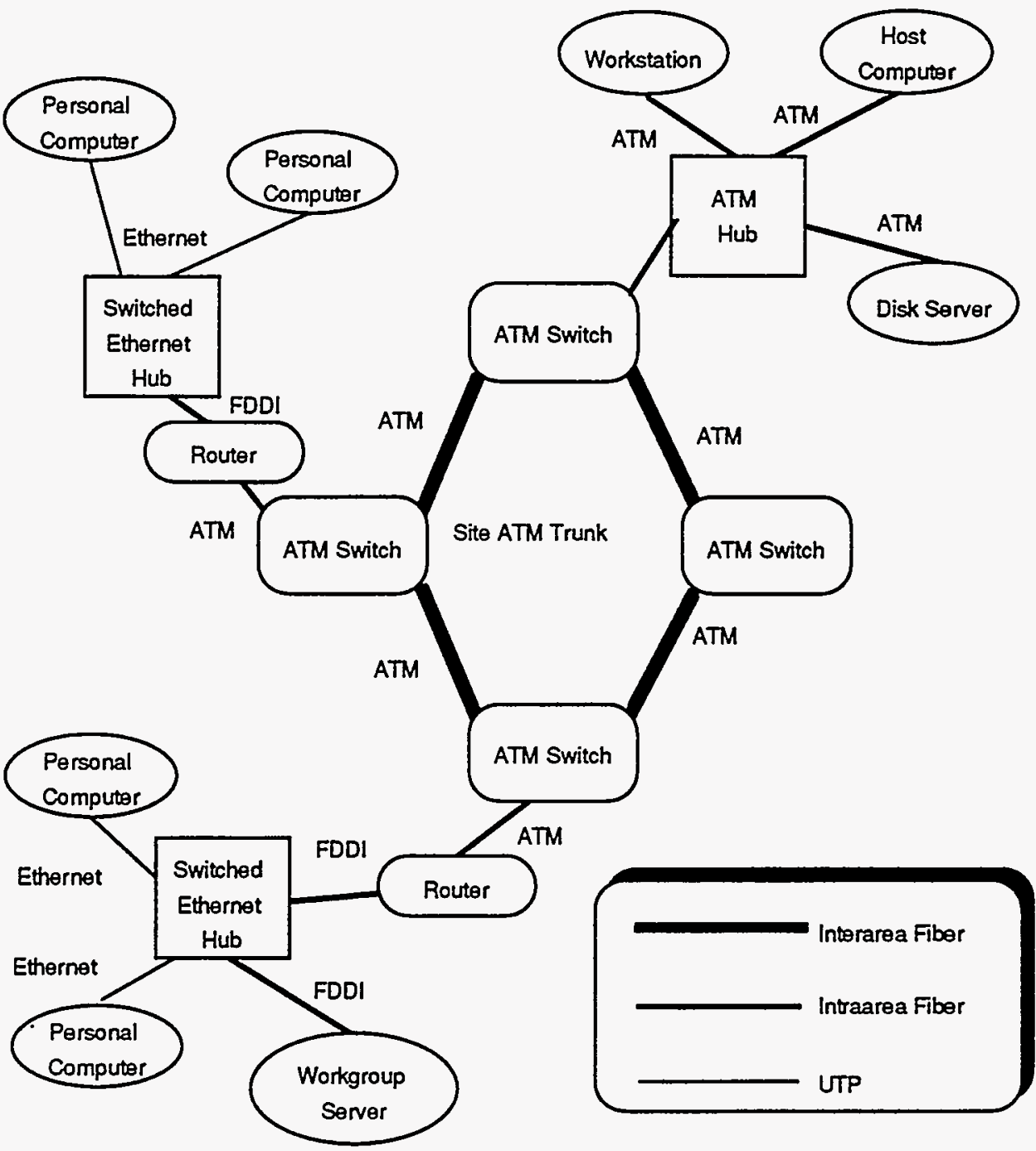

Figure 2: The network of the future. 


\section{Starting Point}

The Savannah River Site's primary data communications network is called SRSnet. SRSnet is comprised of facility or area Ethernet LANs interconnected by digital telephone circuits (T1) or by the site broadband (CCTV) system. Additionally, two Fiber Distributed Data Interface (FDDI) high performance local area networks have been established as part of SRSnet, one as a trunk facility joining together Ethernet segments and one as a high speed LAN for Central Computer Facility (CCF) system access. Figure 3 shows an overview of the SRSnet.

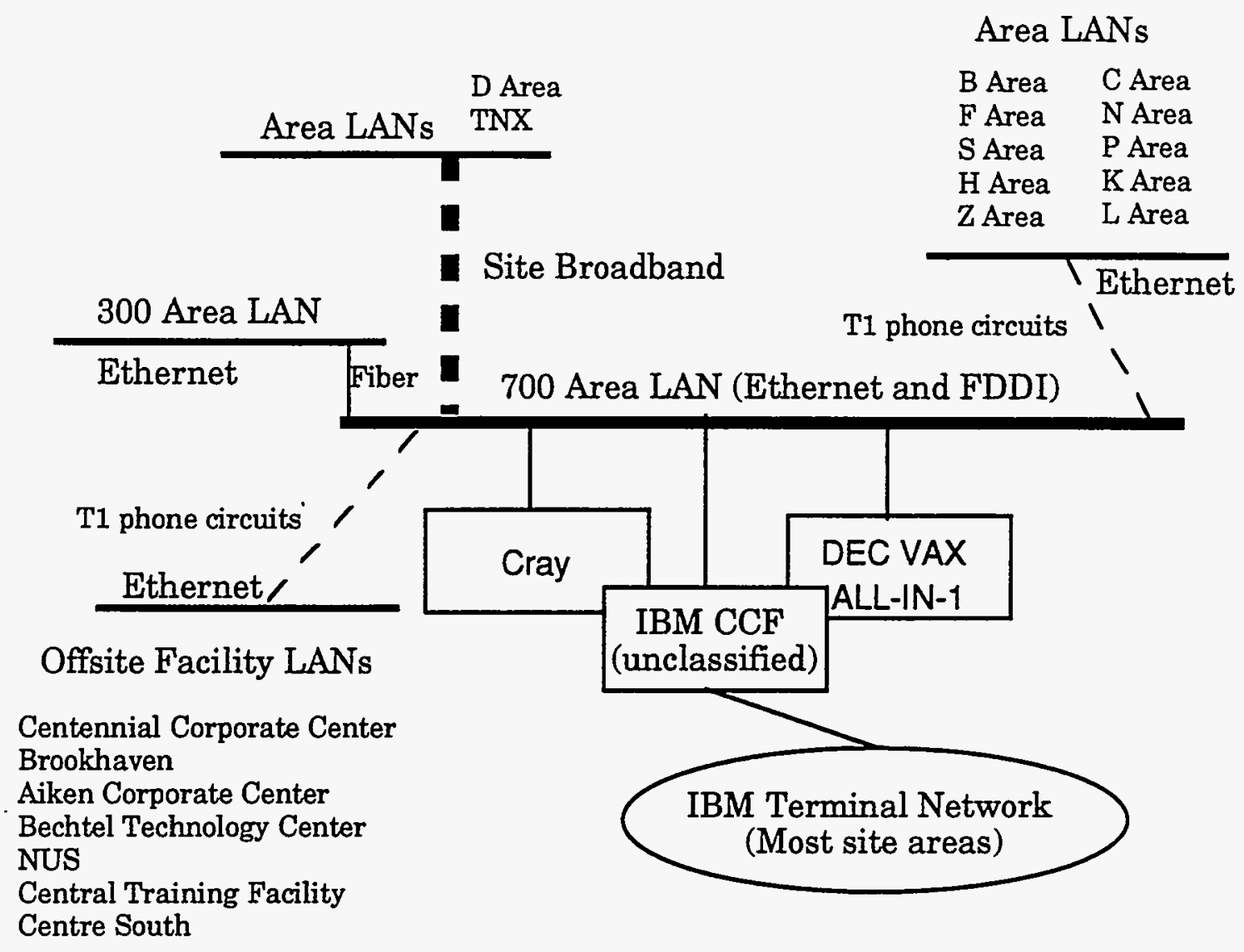

Figure 3: SRSnet overview.

All new Ethernet installations are being installed using the 10BaseT standard for Ethernet. This provides for Ethernet LANs over data grade unshielded twisted pairs (UTP). The site standard for UTP, locally called "universal wiring," consists of multiple UTP data ports per work location. The UTP wiring is terminated in a 10BaseT communications hub located in an equipment room. The 10BaseT hubs are attached to SRSnet, often using fiber optics cables between buildings in a given area or facility. All new on- and offsite buildings are being universally wired as a routine part of building construction. Additionally, universal wiring and 10BaseT connectivity are being retrofitted to existing site buildings on network upgrade projects.

Most multi-user systems (typically VAX, UNIX, and IBM hosts) attach to the LAN at Ethernet speeds (10 million bits per second), though the Cray and the IBM Central Computer Facility attach to the LAN at FDDI speeds ( 100 million bits per second). However, a majority of the site's personal computer systems attach to the LAN via slow speed devices called terminal servers. 
These permit LAN access at speeds up to 19,200 bits per second. Most of the existing onsite building wiring is based on old technology and will support only these slower data rates.

One IRM project, WISDOM, has been encouraging attachment of personal computers directly to the LAN at Ethernet data rates. Under current WISDOM implementations, some 100-200 workstations share a common Ethernet. Although each of the workstations has the capability to generate 1 to 5 million bits per second (mbps) on the LAN, in practice, LAN utilization rates have remained below capacity as the host-based applications commonly used today generate relatively low amounts of data traffic. Thus, the use of WISDOM in a facility requires the facility to be universally wired.

There are no current installations of wireless LANs at the site.

The site network can be judged according to the three criteria used to evaluate the required network of the future:

The network must be reliable.

Today, the LAN portions of the SRSnet are reasonably reliable. However, there is little redundancy built into the system, and key components can and do occasionally fail. The major threat to reliability is the lack of good proactive network management tools to detect component failure and limit resulting downtown.

The Wide Area Network (WAN) components of SRSnet, the digital and analog communications facilities leased from Southern Bell to link onsite areas and WSRC offsite facilities together are not as reliable as the LAN components. This is especially true of the site broadband (CCTV) system used to support TNX and D areas.

The network must be robust.

SRSnet, consisting mainly of 10 million bits of information per second LANs and 1.5 mbps WAN links, is robust enough for host-based (terminaloriented) applications, since these types of applications sustain only small data transfer requirements.

The typical LAN user segment today is shared media Ethernet so that every user on that segment (typically 100 to 200 directly attached workstations) shares equally in its use. A single pair of personal computers today can utilize up to $50 \%$ of an Ethernet's capability in a file transfer. As applications such as multi-media documents, computer based-training, and client/server database queries are developed, the network workstations will utilize this capability to saturate the network to the point that network reliability is endangered.

Just as the LAN links of today are inadequate for the demands of tomorrow, the WAN links are also too slow for demands that will be placed on it. Ideally, the network should present the appearance to application developers and users of a utility that works equally well across all locations. Today, care must be exercised in the design and use of network-intensive applications that cross wide area boundaries.

There must be network-wide user authentication.

There is no network-wide authentication system in use at SRS today. Though computer ID format has been standardized across site systems, 
each system maintains its own list of user IDs and associated passwords and requires the user to authenticate (that is, $\log$ in) himself or herself for service. 


\section{Networks Infrastructure Milestones}

The following infrastructure milestones must be resolved to adopt the technology. Each is described in more detail in the following sections.

Networks.I.1 Install the Replacement Telephone System

Networks.I.2 Complete Intra-area Cabling

Networks.I.3 Complete Site Universal Wiring

Networks.I.4 Complete Building LANs

Networks.I.5 Install Ethernet/FDDI Network Trunks

Networks.I.6 Install Switched Ethernet Hubs

Networks.I.7 Install ATM Network Trunks

Networks.I.8 Install ATM Workgroup Networks

Networks.I.9 Complete Network Management System

Networks.I.10 Initiate Network Authentication Service

Networks.I.11 Install Wireless LANs as Extension to SRSnet

Networks.I.12 Complete Analysis of Network, Telephone, and Dedicated Fiber Usage Requirements

Networks.I.13 Provide Access Between SRSnet and Offsite Locations 


\section{Networks.I.1 Install the Replacement Telephone System}

Responsible Organization

IRM

Initiation Date

$1 Q / 94$
Sponsoring Organization

WSRC

Status

Project started

\section{Anticipated Duration}

24 months

\section{Resources Considerations}

RTS implementation resource requirements have been identified and allocated.

\section{Discussion}

The Replacement Telephone System (RTS) is installed with supporting inter- and intra- area fiber optic cabling. Inter-area fiber (single mode) will connect all site areas together. Implementers of SRSnet can utilize this fiber either directly (via data network interfaces to FDDI or ATM technologies) or indirectly (via the use of high speed [T3 at $45 \mathrm{mbps}$ ] telephone circuits). Intra-area fiber (multimode) can be utilized for Ethernet, FDDI, and ATM connections within the area.

\section{Action Items}

1) Have vendor install RTS, including supporting inter-area and intra-area fiber cables.

2) Choose interface technology between RTS and LAN is chosen.

3) Add interfaces between RTS and LAN to provide improved inter-area data transport.

\section{Dependencies}

None

\section{Benefits}

The RTS provides fiber optic cables connecting all site areas and various facilities within each area. Various LAN and WAN technologies can utilize this fiber at speeds of up to hundreds of megabits per second today, and billions of bits per second in the future. This milestone enables the completion of the intra-area cabling, universal wiring, and current and future network trunks, as well as ATM workgroups. 


\section{Networks.I.2 Complete Inter-area Cabling}

Responsible Organization

IRM

Recommended Initiation Date

$1 \mathrm{Q} / 94$

Anticipated Duration

24 months

\section{Resource Considerations}

Most new intra-area cabling will consist of fiber provided by Replacement Telephone System (RTS). Additional funding requirements expected to be minimal.

\section{Discussion}

Installation of fiber optic or coax cable that connects each facility (building or trailer) in a given SRS area or WSRC offsite location will be part of the RTS. Additional funding will be required for special connection requirements.

\section{Action ltems}

1) Identify intra-area cabling requirements.

2) Reconcile requirements with RTS activities.

3) Initiate additional cabling projects and complete them where needed.

\section{Dependencies}

\section{Networks.I.1}

Networks.A.1

Install the Replacement Telephone System

Set Site Direction, Funding, and Priority

\section{Benefits}

This milestone provides the prerequisite inter-area cable plant upon which all SRS area LANs will be constructed. Fiber optic cabling provides great growth potential. Current implementation at 10 mbps will be upgraded to much faster data rates (on the existing cables) in the future. This milestone enables the milestones concerning installation of current and future network trunks (Ethernet, FDDI, and ATM). 


\section{Networks.I.3 Complete Site Universal Wiring}

Responsible Organization

IRM

Initiation Date

$1 \mathrm{Q} / 94$

\section{Sponsoring Organization}

IRM

Status

All major offsite locations were wired as part of facility preparation. Wiring projects for several onsite buildings have been completed or are in progress. The degree of installation of this wiring is inconsistent across the site.

\section{Anticipated Duration}

24 months

\section{Resource Considerations}

A universal wiring drop costs on average from $\$ 250$ (new buildings) to $\$ 800$ (old buildings). Currently, wiring is two-thirds complete. The Replacement Telephone System (RTS) project will support most of the effort to complete the wiring as part of WSRC's contract with the RTS vendor.

\section{Discussion}

Universal wiring is the installation of unshielded twisted pairs (UTP) of data-grade copper wire according to site standards for facility wiring in each SRS building and all WSRC offsite locations. Wiring being installed today (Category 5 wire) supports the 10BaseT standard for Ethernet LANs as well as future standards for FDDI and ATM technologies over UTP.

\section{Action ltems}

1) Identify and prioritize building wiring requirements.

2) Determine funding mechanism by project.

3) Initiate wiring projects and complete them where funded.

\section{Dependencies}

Networks.A.1 Set Site Direction, Funding, and Priority

\section{Benefits}

This milestone provides the necessary building wiring upon which the user access segments of SRSnet will be constructed. UTP wire cabling provides good growth potential. Current implementation at 10 mbps may be upgraded to much faster data rates (up to $155 \mathrm{mbps}$ ) in the future. This milestone enables milestones for current Ethernet installations as well as switched Ethernet hub installation and ATM workgroup implementations. 


\section{Networks.I.4 Complete Building LANs}

Responsible Organization

IRM

Initiation Date

$1 \mathrm{Q} / 94$

\section{Anticipated Duration}

24 months

\section{Resource Considerations}

Equipment required to complete the second third of this activity is currently on hand. Additional network connections expected to cost an additional $\$ 3$ million.

\section{Discussion}

Completion of the site building LANs involves the installation of Ethernet hub equipment, LAN adapters in SRS personal computers, and supporting LAN interconnect devices (bridges and repeaters) required to complete Ethernet LANs for all WSRC/BSRI work locations. This will provide each work location at least one 10BaseT Ethernet port for connecting devices to SRSnet and the means to connect personal computers to the Ethernet ports.

\section{Action ltems}

1) Identify work locations and special requirements.

2) Establish funding mechanism for obtaining network equipment and implementation priorities.

3) Install LAN hubs and bridges to support these requirements.

4) Install LAN adapters in user personal computers.

\section{Dependencies}

\section{Networks.I.3}

Networks.A.1

Complete Site Universal Wiring

Set Site Direction, Funding, and Priority

\section{Benefits}

This milestone provides the basic Ethernet connection capability for every SRS work location, establishing access to a building or workgroup LAN operating at $10 \mathrm{mbps}$. 


\title{
Networks.I.5 Install Ethernet/FDDI Network Trunks
}

\section{Responsible Organization}

IRM

Initiation Date

$1 \mathrm{Q} / 94$

\section{Anticipated Duration}

36 months

\section{Resource Considerations}

Major equipment to support network trunks are network routers and bridges. Although there is an existing inventory of this gear to complete the site's Ethernet trunks, upgrades to this equipment will require additional capital to convert Ethernet trunks to FDDI. These upgrades will be driven by network traffic and utilization rates.

\section{Discussion}

This milestone provides for the installation of network connection equipment (routers and bridges) to utilize existing and future fiber optic or coax cables that connect each facility (building or trailer) in a given SRS area or WSRC offsite location. Intra-area and inter-area cabling will support both FDDI and Ethernet trunks. Choice of which to use in a particular situation requires a cost/performance calculation. Over time, all trunks will be upgraded from Ethernet to FDDI (or ATM) as the faster technology becomes cost effective and user data transport requirements grow.

\section{Action Items}

1) Identify intra-area network trunk topology requirements.

2) Identify additional equipment required.

3) Establish funding and purchase additional equipment.

4) Install network routers and bridges to support these requirements.

\section{Dependencies}

\author{
Networks.I.2 Complete Inter-area Cabling \\ Networks.I.3 Complete Site Universal Wiring \\ Networks.I.4 Complete Building LANs \\ Networks.A.1 Set Site Direction, Funding, and Priority
}

\section{Benefits .}

This milestone provides the prerequisite intra-area network facilities upon which all SRS area LANs will be constructed. It is dependent on having physical cabling in place and provides the devices that utilize the cabling to provide robust LAN facilities. This will be the starting point from which all upgrades to network capabilities will follow. 


\section{Benefits}

This milestone provides an upgrade path for the workgroup segments of SRSnet. User workstations, LAN adapters, and network wiring need not be upgraded to complete this milestone. Each user on a switched Ethernet hub (SEH) gains a dedicated $10 \mathrm{mbps}$ channel to every other user in the workgroup-ideal for certain multi-media applications. Access to shared servers is provided by.FDDI or ATM interfaces built into the SEH. 


\section{Networks.I.7 Install ATM Network Trunks}

\section{Responsible Organization}

IRM

Initiation Date

$1 Q / 96$

\section{Sponsoring Organization}

IRM

\section{Status}

Technology under study. Replacement Telephone System vendor promises ATM capabilities.

\section{Anticipated Duration}

48 months

\section{Resource Considerations}

ATM is new technology and very expensive today. Fully converting the site inter-area network trunk to ATM could cost as much as $\$ 1$ to $\$ 2$ million in capital spending. Conversion of the each area's LAN trunk to ATM could cost nearly as much, depending on area size and network complexity. These costs will certainly come down by the time the site is ready to go to ATM.

\section{Discussion}

This milestone provides an upgrade path for the site inter-area and intra-area network facilities upon which all SRS area LAN's are constructed. It is dependent on having physical cabling (inter- and intra-area fiber) in place and provides the devices that utilize the cabling to provide robust LAN facilities. In some cases, existing devices will be upgraded to support ATM; in other cases, new devices (switches and routers) will be required to add ATM capability.

\section{Action Items}

1) Define network trunk data requirements.

2) Pilot technology between two site areas.

3) Establish funding for network upgrade to ATM.

4) Install ATM switches and upgrades to network routers to support these requirements.

\section{Dependencies}

$\begin{array}{ll}\text { Networks.I.2 } & \text { Complete Inter-area Cabling } \\ \text { Networks.I.3 } & \text { Complete Site Universal Wiring } \\ \text { Networks.A.1 } & \text { Set Site Direction, Funding, and Priority } \\ \text { Networks.A.2 } & \text { Relocate Most Network Users onsite } \\ \text { Networks.T.2 } & \text { Complete ATM Standards } \\ \text { Networks.T.3 } & \text { Complete ATM Interfaces for Routers/Hubs }\end{array}$




\section{Benefits}

Fully implemented, this milestone would support all identified future network requirements and improve network robustness, as ATM technology works equally well between site areas as within a building. 


\section{Networks.I.8 Install ATM Workgroup Networks}

\section{Responsible Organization}

IRM

Initiation Date

$4 \mathrm{Q} / 95$
Sponsoring Organization

WSRC

Status

Technology under study

\section{Anticipated Duration}

48 months

\section{Resource Considerations}

ATM workstation connections cost approximately $\$ 10,000$ per workstation today. This is very new technology, and the price is projected to fall below $\$ 1,000$ per workstation within two years.

\section{Discussion}

This milestone provides the ability to link high performance workstations or host computers/servers into a LAN segment with very high performance. Today, vendors are shipping hubs and ATM interfaces at $155 \mathrm{mbps}$, but equipment at $620 \mathrm{mbps}$ is promised for the near future. This would provide a good upgrade path for the CCF FDDI ring, as well as for situations (scientific visualization) requiring high bandwidth. The installation of workgroup local area networks based on ATM technology consists of ATM adapters in workstations, ATM local switching hubs, and data-grade UTP or fiber optic cabling between workstation and hubs. Data-grade UTP will support most applications, but fiber cabling will be required for those applications needing more than 155 mbps.

\section{Action Items}

1) Define high performance LAN requirements defined.

2) Install ATM hubs and upgrades to network interfaces on host systems, servers, and workstations to support these requirements.

3) Connect ATM workgroup to site ATM switching fabric.

\section{Dependencies}

Networks.I.2

Complete Inter-area Cabling

Networks.I.3

Complete Site Universal Wiring

Networks.T.2

Complete ATM Standards

Networks.T.4

$$
\text { Complete ATM Interfaces for Workstations/Host Computers }
$$

\section{Benefits}

This milestone provides the high speed and dedicated bandwidth required to build very high performance workstation networks. The key components of ATM are or will soon be standardized to provide the benefits of support by all key systems and communications vendors. 


\section{Networks.l.9 Complete Network Management System}

\section{Responsible Organization}

IRM

Initiation Date

$1 \mathrm{Q} / 94$

\section{Anticipated Duration}

36 months

\section{Resource Considerations}

Hardware and software to support a Network Management System (NMS) has been obtained. Greatest resource requirements involve dedicating network analyst(s) to customizing and integrating purchased NMS components and the continued commitment to keep network documentation current and entered in the NMS. Additional NMS components may be required, but these would not represent a large capital or cost investment.

\section{Discussion}

A comprehensive NMS is a key to creating a robust network. It gives network operators the ability to detect problems and device failures early and to diagnose and solve network error conditions remotely. Completion of a comprehensive and proactive network and networkattached resource management platform requires integrating network management capabilities supplied by several vendors and documenting the current network down to user-device attachments.

\section{Action ltems}

1) Purchase integrating Network Management System (NMS).

2) Integrate product-specific management information (MIB) into NMS.

3) Set up network event alerts to include loss of key host systems or servers.

4) Integrate NMS access software into personal computers to allow remote queries/sets.

\section{Dependencies}

Netsys.I.3

Netsys.I.12

\section{Benefits}

Ensure Broad Availability of Networked Workstations

Implement Reliable Performance Monitoring

A comprehensive NMS reduces or eliminates network down time and provides the data necessary to improve the robustness of the network. 


\section{Networks.I.10 Initiate Network Authentication Service}

\section{Responsible Organization}

IRM

Initiation Date

$1 Q / 95$

Anticipated Duration

36 months

\section{Resource Considerations}

A migration to network-based authentication is primarily a software issue, as operating system, workstation, and data access software will have to be upgraded to support network authentication. A number (possibly one per site area) of security servers will be required as well. These could be relatively low cost $(\$ 20,000)$ high performance workstations. Major cost component could be licensing security client software on all site workstations.

\section{Discussion}

Network authentication provides users with network-transparent computing. That is, a user is able to authenticate him- or herself once and gain access to various network services and data without further authentication. This helps provide the effect that there is a single location for services and data, the network. This milestone provides for conversion of system-based user authentication to network-based authentication. This requires network authentication server(s) and modification of host/server operating systems to support network-based authentication protocols.

\section{Action Items}

1) Perform laboratory-scale testing based on early DCE products.

2) Work with key vendors to attempt to influence DCE development.

3) Install DCE-compliant operating systems as they become available.

4) Install redundant network authentication servers.

\section{Dependencies}

Netsys.I.3

Netsys.P.1

Networks.A.4

Networks.T.5

Netsys.I.12
Ensure Broad Availability of Networked Workstations Implement Single User Login to Network Obtain Computer Security Endorsement of Network-based Authentication Make DCE Security Services Available in Key Environments Implement Reliable Performance Monitoring

\section{Benefits}

Network authentication greatly eases system administration tasks, as each user need be entered in only one comprehensive authentication database. Greatest benefit is simplified operations for the network user and transparent access to data for which the user is authorized. 


\section{Networks.I.11 Install Wireless LANs as Extension to SRSnet}

Responsible Organization

IRM

Initiation Date

$4 Q / 94$
Sponsoring Organization

IRM

Status

Concept being studied

\section{Anticipated Duration}

24 months

\section{Resource Considerations}

Widespread adoption of wireless LAN devices is not anticipated. Rather, this technology is proposed for special situations where wired-LANs are inadequate. Cost per wireless connection expected to be in the $\$ 500$ per connection range.

\section{Discussion}

The capability to extend the LAN with wireless technology provides cost effective network connections in those situations where rewiring a facility is cost prohibitive or restricted for other reasons. Wireless technology also supports the concept of mobile computing (that is, the ability to utilize a workstation from anywhere on the site, in the local area, or even in the world to connect up to services and data sources provided by SRSnet).

\section{Action ltems}

1) Conduct laboratory-scale testing of wireless components.

2) Pilot in SRS production area.

3) Develop guidelines on when and where to use wireless rather than wired solutions.

4) Complete limited production implementation.

\section{Dependencies}

Networks.A.3 Resolve Security and Spectrum Management Issues for Wireless LAN

\section{Benefits}

The installation of wireless LAN connections provides network users with services equivalent to those enjoyed by wired users. This technology will permit extension of the network to areas not feasible for wiring. 


\section{Networks.l.12 Complete Analysis of Network, Telephone, and Dedicated Fiber Usage Requirements}

\section{Responsible Organization}

IRM

Initiation Date

$4 \mathrm{Q} / 94$

\section{Anticipated Duration}

24 months

\section{Resource Considerations}

This milestone requires no hardware or software acquisitions, but will require a limited amount of IRM manpower to complete.

\section{Discussion}

Multimedia and other applications will require the ability to transfer data (mostly digital but some analog such as voice and video) across the organization at increasingly faster rates. New capabilities are planned for the site network and telephone system (including dedicated fiber optic cables between buildings and areas) that provide a great increase in current capabilities to accomplish this task. A use/capabilities study is required to gain a more detailed understanding of the new data transfer requirements and the best way to meet these needs.

\section{Action ltems}

1) Identify/monitor multimedia capabilities embedded in new telephone system and new cabling.

2) Evaluate SRSnet capabilities to transfer data across various locations and determine limitations.

3) Design multimedia and other high bandwidth applications to take advantage of the strengths of the infrastructure.

4) Propose necessary changes to the infrastructure to support new application requirements.

\section{Dependencies}

None

\section{Benefits}

SRS will optimize usage of the data network, the new telephone system, and direct fiber delivery for multimedia and other high bandwidth applications, and will avoid duplication of services between LAN, direct fiber, and analog delivery of data. 


\section{Networks.I.13 Provide Access Between SRSnet and Offsite Locations}

Responsible Organization

IRM

Initiation Date

$3 Q / 94$

\section{Anticipated Duration}

18 months
Sponsoring Organization

IRM

Status

Concept being studied

\section{Resource Considerations}

A limited amount of hardware and/or software will be required to implement gateways to offsite locations. Special requirements may require significant IRM staff resources to implement, especially security-related requirements.

\section{Discussion}

SRSnet today has limited ability for access from offsite and for access to offsite information resources by SRS employees. Security and operational issues will be addressed to improve this capability so that site employees can access their electronic mail from offsite, outside organizations can access selected SRS data, and SRS employees can gain ready access to information stored on worldwide network facilities.

\section{Action ltems}

1) Identify requirements for offsite access.

2) Develop technology plan to meet these requirements.

3) Pilot offsite access on small scale.

4) Extend pilot to all SRS employees.

\section{Dependencies}

Networks.A.5 Resolve Security and Network Use Issues for Offsite Access to and from SRSnet

\section{Benefits}

New SRS mission requirements are driving the needs for SRS employees to gain access to offsite information and for others (DOE, universities, suppliers, etc.) to gain access to selected SRS data. Allowing employees to access their electronic mail from home or when traveling offsite will improve site productivity through improved communications. 


\section{Networks Administrative Milestones}

The following administrative milestones must be resolved to adopt the technology. Each is described in more detail in the following sections.

Networks.A.1 Set Site Direction, Funding, and Priority

Networks.A.2 Relocate Most Network Users Onsite

Networks.A.3 Resolve Security and Spectrum Management Issues for Wireless LAN

Networks.A.4 Obtain Computer Security Endorsement of Network-based Authentication

Networks.A.5 Resolve Security and Network Use Issues for Offsite Access to and from SRSnet 


\section{Networks.A.1 Set Site Direction, Funding, and Priority}

Responsible Organization

ALD

Initiation Date

$1 \mathrm{Q} / 94$

\section{Anticipated Duration}

12 months

\section{Resource Considerations}

Management (both IRM and WSRC) commitment is required to establish the appropriate levels of support for establishing and maintaining the site network as a valuable site resource.

\section{Discussion}

Establishment of site policy and practices to support central direction, funding, and priority for site information technology initiatives (I/T).

\section{Action ltems}

1) Activate SRS Computing Council to establish site $I / T$ priorities.

2) Endorse site Strategic Plan for $I / T$.

3) Develop IRM cost estimates and timing required for site network initiatives for Computer Council approval.

\section{Dependencies}

None

\section{Benefits}

Completion of the site network is a significant site infrastructure issue. Current and future mission assignments require a robust and reliable network that is uniformly implemented across SRS. Without central direction and funding, network implementations tend to be uneven, as certain departments or facilities see the need to fund the network and others do not. 


\section{Networks.A.2 Relocate Most Network Users Onsite}

\section{Responsible Organization}

SRS Management

Initiation Date

$1 Q / 94$

\section{Anticipated Duration}

60 months

\section{Resource Considerations}

New onsite office space will be universally wired as part of construction. As users abandon offsite facilities, network equipment can be recovered and utilized in onsite locations.

\section{Discussion}

Most SRS employees currently located in office facilities in Aiken, Augusta, and other surrounding locations will return to onsite facilities. Because of the fiber network being installed as part of the Replacement Telephone System (RTS), onsite communications potential is nearly unlimited. Inter-area communications rates of $100 \mathrm{mbps}$ will be established initially, a major improvement in the $1.5 \mathrm{mbps}$ rate used to link offsite facilities today.

\section{Action Items}

1) Designate employees to move by site management.

2) Install network in new onsite facilities.

3) Remove network lines and support equipment for abandoned offsite locations.

\section{Dependencies}

None

\section{Benefits}

With most employees onsite, network implementations are not subject to use of tariffed circuits from the phone company. High speed links between areas become much more economical over site-owned communications facilities, especially site inter-area fiber optic cable. Anyone left offsite will necessarily have reduced levels of network service. 


\section{Networks.A.3 Resolve Security and Spectrum Management Issues for Wireless LAN}

Responsible Organization

IRM

Initiation Date

$2 Q / 94$
Sponsoring Organization

IRM

Status

Under way

\section{Anticipated Duration}

24 months

\section{Resource Considerations}

IRM will work with Security to investigate the technology and run pilot wireless networks to prove security.

\section{Discussion}

Establishment of site policy and practices on the use of wireless communications that address security concerns as well as avoidance of frequency allocation problems in the radio frequency spectrum used by the most effective wireless LANs.

\section{Action Items}

1) Establish Wireless Working Group to include IRM, Security, and DC\&S.

2) Develop Wireless Policy and Guidelines.

\section{Dependencies}

None

\section{Benefits}

The capability to extend the LAN with wireless technology provides cost-effective network connections in those situations where rewiring a facility is cost prohibitive or restricted for other reasons. Security and frequency management concerns must be addressed prior to any wireless implementations. 


\section{Networks.A.4 Obtain Computer Security Endorsement of Network- based Authentication}

Responsible Organization

SSP

Initiation Date

$2 \mathrm{Q} / 94$

Anticipated Duration

24 months

\section{Resources Considerations}

IRM-sponsored pilot implementations will be required.

\section{Discussion}

Establishment of site policy, procedures, and guidelines acceptable to site security organizations for the use of network-based user authentication and access control.

\section{Action ltems}

1) Pilot a limited project to demonstrate security features of network authentication.

2) Develop network authentication policy, procedures, and guidelines.

\section{Dependencies}

None

\section{Benefits}

Network authentication provides users with network-transparent computing. That is, a user is able to authenticate him or herself once and gain access to various network services and data without further authentication. This helps provide the effect that there is a single location for services and data, the network. 


\section{Networks.A.5 Resolve Security and Network Use Issues for Offsite Access to and from SRSnet}

Responsible Organization

SSP

Initiation Date

$2 \mathrm{Q} / 94$

Anticipated Duration

24 months

\section{Resource Considerations}

IRM participation with Security in team to study issues and make recommendations.

\section{Discussion}

Establishment of site policy, procedures, and guidelines acceptable to site security organizations and site management for the access of selected site information resources from offsite and improving the access of offsite information resources for site personnel.

\section{Action ltems}

1) Develop study to define offsite access requirements.

2) Conduct limited pilot project to demonstrate security features of offsite access.

3) Develop network access policy, procedures, and guidelines.

\section{Dependencies}

None

\section{Benefits}

New SRS mission requirements are driving the need for SRS employees to gain access to offsite information and for others (DOE, universities, suppliers, etc.) to gain access to selected SRS data. Allowing employees to access their electronic mail from home or when traveling offsite will improve site productivity through improved communications. 


\section{Networks Technology Milestones}

The following technology milestones must be resolved to adopt the technology. Each is described in more detail in the following sections.

Networks.T.1 Implement Cost-effective Switched Ethernet Hubs

Networks.T.2 Complete ATM Standards

Networks.T.3 Complete ATM Interfaces for Routers/Hubs

Networks.T.4 Complete ATM Interfaces for Workstations/Host Computers

Networks.T.5 Make DCE Security Services Available in Key Environments 


\section{Networks.T.1 Implement Cost-effective Switched Ethernet Hubs}

Responsible Organization

N/A

Initiation Date

$1 Q / 94$
Sponsoring Organization

N/A

\section{Status}

Initial switched Ethernet products became available during 1993 for approximately $\$ 1,500$ per port. Follow-on products being announced at approximately $\$ 500$ per port.

\section{Anticipated Duration}

24 months

\section{Resource Considerations}

Minimal effort required to follow development of new network technology.

\section{Discussion}

The availability of switched Ethernet hubs (SEH) with price per port costs that approximate current shared Ethernet hub prices ( $\$ 150$ per port) is the ultimate goal. Today's higher performance SEHs cost more than traditional hubs. Early production implementations at SRS are expected to be a hybrid approach, putting several users on a dedicated Ethernet port.

\section{Action ltems}

1) Conduct laboratory pilot for proof of concept.

2) Follow and influence vendor developments.

3) Procure new site standard for SEH when price becomes attractive.

\section{Dependencies}

None

\section{Benefits}

This milestone provides the necessary technology for an upgrade path for the workgroup segments of SRSnet. User workstations, LAN adapters, and network wiring need not be upgraded to complete this milestone. Each user on a switched Ethernet hub (SEH) gains a dedicated $10 \mathrm{mbps}$ channel to every other user in the workgroup-ideal for certain multimedia applications. Access to shared servers is provided by FDDI or ATM interfaces built into the SEH. 


\section{Networks.T.2 Complete ATM Standards}

Responsible Organization

N/A

Initiation Date

$1 Q / 94$

\section{Sponsoring Organization}

N/A

Status

Various national, international, and vendor bodies are considering the definition of the interoperability standards for building ATMbased communications networks.

\section{Anticipated Duration}

36 months

\section{Resource Considerations}

Minimal effort required to follow development of new network technology.

\section{Discussion}

ATM-related standards must be completed to allow the completion of heterogeneous wide area and local area data networks based on ATM technology. An active vendor alliance, the ATM Forum, is proving more effective than formal standards organization in identifying and resolving ATM interoperability issues. Formal standards are expected to codify the work of the ATM Forum.

\section{Action ltems}

1) Follow industry ATM standards efforts.

2) Encourage vendors to promote and follow standards.

\section{Dependencies}

None

\section{Benefits}

This milestone is required before the site can build multi-vendor ATM trunks as illustrated in Figure 2 of this Roadmap. Without standards, early implementations of private ATM switches and interface devices are limited to single vendor solutions. ATM standards have been proposed for lower speed ATM networks (155 mbps or less) over UTP. 


\section{Networks.T.3 Complete ATM Interfaces for Routers/Hubs}

Responsible Organization

N/A

Initiation Date

$4 \mathrm{Q} / 95$
Sponsoring Organization

N/A

Status

Most LAN router and hub vendors have released product plans detailing expectations to produce ATM interfaces in the future.

\section{Anticipated Duration}

12 months

\section{Resource Considerations}

Minimal effort required to follow development of new network technology.

\section{Discussion}

ATM hardware and software interfaces are required for communications routers and LAN hubs in use at SRS to provide a high-speed connectivity option for these devices. Early (and costprohibitive) implementations are becoming available.

\section{Action Items}

1) Follow industry ATM product development efforts.

2) Encourage key network vendors to develop products that provide SRS a cost-effective transition path to ATM.

\section{Dependencies}

Networks.T.2 Complete ATM Standards

\section{Benefits}

This milestone is required before the site can build multi-vendor ATM trunks as illustrated in Figure 2. Currently, it appears that existing (high-end) network routers at SRS will have an upgrade path to ATM, but it is uncertain whether there will be an upgrade migration to ATM for existing hubs. 


\section{Networks.T.4 Complete ATM Interfaces for Workstations/ Host Computers}

Responsible Organization

N/A

Initiation Date

$4 Q / 94$
Sponsoring Organization

N/A

Status

Various (vendor-specific) interfaces are available today for UNIX-based workstations. Other system vendors have announced plans for products.

\section{Anticipated Duration}

12 months

\section{Resource Considerations}

Minimal effort required to follow development of new network technology.

\section{Discussion}

ATM hardware and software interfaces are required for workstations, host computers, and servers in use at SRS to provide high speed connectivity options for these systems. Early implementations are becoming available, but these are costly and provide only marginal improvement over FDDI today.

\section{Action Hems}

1) Follow industry ATM product development efforts.

2) Encourage key network vendors to develop products that provide SRS a cost-effective transition path to ATM.

\section{Dependencies}

Networks.T.2 Complete ATM Standards

\section{Benefits}

This milestone is required before the site can build multi-vendor ATM LANs in support of high end workstations, host computers, and servers, as illustrated in Figure 2. 


\section{Networks.T.5 Make DCE Security Services Available in Key Environments}

Responsible Organization

N/A

Initiation Date

$1 Q / 95$

\section{Anticipated Duration}

24 months

\section{Resource Considerations}

Minimal effort required to follow development of new network technology. A DCE demonstration in the laboratory will provide proof of the operational principles of distributed security.

\section{Discussion}

Distributed Computing Environment (DCE) security services provide the standard protocol for network-based user authentication and are supported on the CCF, VAX/VMS, ADX, and the site standard NOS.

\section{Action Items}

1) Follow industry DCE product development efforts.

2) Encourage key network vendors to develop products that provide SRS a cost-effective transition path to DCE-based security services.

3) Follow NOS vendor global directory standards and gateway interface to DCE security.

4) Conduct a laboratory-scale DCE implementation.

\section{Dependencies}

None

\section{Benefits}

This milestone is required before the site can implement network-wide user authentication based on the DCE security services standard. Most large systems vendors plan to support DCE. NOS vendors are generally looking at some sort of gateway approach to provide linkage to DCE-based authentication. 


\section{Networked Systems Roadmap}

\section{Introduction and Destination}

As stated in the SRS Computing Architecture, the future vision of computing at the Savannah River Site is "standards-based, data-driven, and workstation-oriented, with larger systems being utilized for the delivery of needed information to users in a client-server relationship." Just as the implementation of host-based applications required a robust, secure, and consistent platform, the delivery of client/server applications also requires a supporting infrastructure.

Today we depend on an infrastructure of roads and transportation systems, water and sewage systems, and electric power and communications systems. Personal and commercial vehicles utilize the roads, sharing the infrastructure. At the highest level (interstate highway system), we realize the benefit of centralized planning and implementation, especially when we encounter the dreaded "Freeway Ends" signs. At lower levels (perhaps community level), we often find streets that end unexpectedly or change name as they cross other streets. By necessity, there is a connection between the interstate system and local roads. If the computing infrastructure is to serve the needs of the enterprise, it must be planned and implemented as a total system.

Networked systems can be defined as an infrastructure which includes site network components, protocols, topology, administrative practices, workstations, peripherals, various server systems (such as file and information servers), document manipulation, interpersonal messaging, groupware, etc. A robust communications infrastructure must be established and maintained to leverage the true power of workstations through client/server computing and to support rapid access to large amounts of data. Through standardization, workstations and file servers will also become an integral part of the platform infrastructure to provide the workstation connectivity, manageability, and usability features which all applications will require.

The basic objective of networked systems is to maximize the benefits of personal computing by providing standardization and central management, workstation inter-operability, data interchange, user support, and operating freedom, while reducing the overall cost of ownership. The system encourages the interchange of data regardless of user location or type of computer equipment. Most importantly, this new system provides a well-defined platform upon which applications may be built and an environment flexible enough to adapt to the inevitable changes in the computing industry and Savannah River Site missions. By its nature, an infrastructure must attempt to accommodate diverse needs, necessitating some compromises.

When completed, networked systems should provide the following functionality:

Provide access to knowledge for business decision-making.

Information which is not easily accessible to the correct people at the correct location, and which is not both trustworthy and private, cannot be used in the business decision-making process. Data and applications must be reliably available from all locations. This means that there should be sharable workstations at most geographic locations and that workstations must be capable of accessing user data which is stored in the networked system rather than at individual workstations.

This data must be quickly and easily accessible by those people authorized to access it and yet be protected from damage by hardware or software 
failure or malicious action. This assumes improved data integrity and security with routine backup of user data located on file servers, virus protection, and disaster-recovery processes.

The use of network-wide authentication means the user authenticates to a security service once, and this service communicates authentication information when required by other service nodes. The directory service aids in synchronizing the information with other networked services.

Provide cost-effective management of personal computing assets.

Networked systems should help the establish standards for the personal computer operating systems, graphical user interfaces, and network interfaces. These standards should provide for diverse user needs and yet encourage extensive use of shared workstations and networked peripherals. The current installed base utilizing the Macintosh OS and DOS/Windows OS will migrate toward future operating systems, but must leverage current assets. Interoperability will be required among all computers via common file and printer sharing and common application software.

A system of hardware asset management and software license management at site or server level rather than individual workstations will be required. Networked systems must provide a delivery vehicle for installation and update of purchased or developed software to file servers and personal computer workstations.

Enable workers or applications to adopt new approaches in business processes.

Networked systems must provide the ability to manage change without impeding it. It must provide mechanisms to increase functionality while reducing administrative overhead.

There must be easy distributed workgroup management with minimized central administration overhead.

\section{Starting Point}

Realizing the importance of controlling the costs of personal computer ownership and the need to effectively utilize the site's investment in computing technology, particularly the large base of personal workstations, the WSRC Information Resource Management Department initiated a pilot program to develop a common networked workstation infrastructure. This pilot system is not a software product, but a system defined by a set of guiding principles. The system is built from commercially available hardware and software components, with custom-developed software where necessary to provide the personal computer management capabilities. An essential part of the design is the ability to adapt to changing site requirements and changing commercial hardware and software products.

Networked systems will be based on the following technologies:

\section{Network Operating System (NOS)}

Today's NOS is server-oriented, creating islands of information and administration. It does not encourage an enterprise view. Movement to an enterprise-oriented NOS with hooks into other network services will provide the basis for building the infrastructure. 
- Graphical User Interface (GUI) Workstations

Apple Macintosh System 7.1 and Microsoft Windows (3.1) currently provide the basis for a graphical user interface (GUI) approach. An IBM (or compatible) PC with a 386 (or better) processor or an Apple Macintosh II (or better) is required to run the client software. Approximately one third of the personal computer systems in use onsite are functionally obsolete and are not capable of running the GUI-required environments. These older machines range in age from 5 to 10 years.

- NOS Global Directory Services

Directory services are provided by the NOS as a service, but must cooperate with other network services such as electronic mail and user authentication services. In addition to the technology itself, organizational or administrative changes will be required to plan and administer the directory. 


\section{Network Systems Infrastructure Milestones}

The following infrastructure milestones must be resolved to adopt the technology. Each is described in more detail in the following sections.

Netsys.I.1

Netsys.I.2

Netsys.I.3

Netsys.I.4

Netsys.I.5

Netsys.1.6

Netsys.1.7

Netsys.I.8

Netsys.I.9

Netsys.I.10

Netsys.I.11

Netsys.I.12
Inștall Robust Client Workstations

Upgrade Workstations to Support Netsys Requirements

Ensure Broad Availability of Networked Workstations

Migrate to New NOS

Initiate NOS Global Directory

Initiate Network Authentication Service

Complete Installation of Territorial File Servers

Manage IP Addresses

Implement Robust File/Print/Application Services

Implement Automatic Software Distribution

Connect Office Peripherals Directly to Network

Implement Reliable Performance Monitoring 


\section{Netsys.l.1 Install Robust Client Workstations}

\section{Responsible Organization}

IRM

Recommended Initiation Date

$1 \mathrm{Q} / 94$
Sponsoring Organization

WSRC

Status

PC upgrade and replacement strategy proposed to DOE as part of WSRC Strategic Plan for Business Computing

\section{Anticipated Duration}

24 months

\section{Resource Considerations}

This milestone requires an ongoing commitment of funds for computer replacement and upgrade. At a five-year turnover rate, this currently amounts to about $\$ 6$ million per year.

\section{Discussion}

Client workstations must have sufficient power and memory to store dynamic tables for field validation by client/server applications, to respond quickly to user inquiries, and to cache frequently used information. At a very minimum, machines more than 5 years old must be replaced. Graphics accelerator cards should be used where needed to provide fast screen painting and refreshing for graphical applications. Sound devices must be available to provide output of speech via headphones.

\section{Action ltems}
1) Establish minimum standard for client workstation.
2) Upgrade or replace all inadequate workstations.
3) Periodically revise standard.

\section{Dependencies}

Netsys.A.1

$$
\text { Set Site Direction, Funding, and Priority }
$$

\section{Benefits}

Inadequate or obsolete workstations will not be acceptable to run sitewide applications, will be difficult or impossible to maintain at the site software standards, and increase the support burden while decreasing employee efficiency. Establishing a centralized policy and procedure will reduce administrative overhead and allow application developers to assume a minimum level of performance. 


\title{
Netsys.I.2 Upgrade Workstations to Support Netsys Requirements
}

Responsible Organization

IRM

Recommended Initiation Date

$2 Q / 94$

\section{Anticipated Duration}

36 months

\section{Resource Considerations}

Much of this work could be outsourced.

\author{
Sponsoring Organization
}

IRM

\section{Status}

Ad hoc upgrades in progress; systematic program required to identify upgrade and complete requirements

\section{Discussion}

Each workstation must have sufficient hardware, proper software, and a network connection to participate in the enterprise network. In some cases, this requires only a reconfiguration of the existing hardware and reloading of software. A plan for upgrade should be prioritized by availability of network file/print services. Once upgraded, the workstation software will be maintained current, the user will access standard applications from the file servers, and the workstation may be easily shared.

\section{Action Items}

1) Determine order of file/print services availability.

2) Assign staff to upgrade hardware/software.

3) Coordinate user training.

\section{Dependencies}

Networks I.3

Netsys.I.1

Netsys.A.1

Netsys.M.1

\author{
Complete Site Universal Wiring \\ Install Robust Client Workstations \\ Set Site Direction, Funding, and Priority \\ Implement PC Utilization Plan
}

\section{Benefits}

This milestone reduces support requirements, automates maintenance of current software, and encourages equipment sharing. Each personal computer is attached directly to the site Ethernet LAN via an adapter card and is assigned a home file server, so that application and communications software can be centralized in the server for access by the client workstations. This requires a degree of standardization in software and hardware configurations on individual personal computers, but greatly simplifies support requirements for personal computer users. This standardization is also required to ensure that applications based on communications between site computers and personal computers function reliably. 


\section{Netsys.l.3 Ensure Broad Availability of Networked Workstations}

\section{Responsible Organization}

IRM

Recommended Initiation Date

$2 \mathrm{Q} / 94$
Sponsoring Organization

IRM

\section{Status}

Effort under way to complete networks and PC utilization plan

\section{Anticipated Duration}

24 months

\section{Resource Considerations}

Implementation of a PC utilization plan in conjunction with procurement will ensure available workstations. Staff should be provided (or outsourcing utilized) to install all new workstations in a standard fashion.

\section{Discussion}

Sitewide applications cannot be delivered until a sufficient user population has access available. Where individuals do not have adequate workstations, shared workstations should be conveniently located.

\section{Action Items}

1) Ensure that supporting network (building and area) in place.

2) Complete personal computer utilization plan.

3) Reconfigure workstations to Netsys standards.

\section{Dependencies}

Networks.I.3

Complete Site Universal Wiring

Netsys.A.1

Set Site Direction, Funding, and Priority

Netsys.A.2

Define Security Principles

Netsys.I.1

Install Robust Client Workstations

Netsys.I.2

Upgrade Workstations to Support Netsys Requirements

\section{Benefits}

This milestone produces a critical mass of standardized clients, making development of sitewide applications cost effective. 


\section{Netsys.I.4 Migrate to New NOS}

Responsible Organization

IRM

Recommended Initiation Date

$2 \mathrm{Q} / 94$

\section{Anticipated Duration}

12 months

\section{Resource Considerations}

Sitewide input and participation during the planning and early implementation may require significant resources. Once established, the resource load should be less than is currently used. The support for the current file servers, if redeployed, would be sufficient.

\section{Discussion}

The implementation of an enterprise NOS produces the infrastructure necessary to provide centralized administration, de-centralized management, and stratified security. Movement away from a server-based NOS toward an enterprise NOS will facilitate the other infrastructure operations.

\section{Action ltems}

1) Design and implement centralized structure.

2) Convert existing client workstations.

3) Install new servers and migrate existing servers.

4) Document security and management procedures.

\section{Dependencies}

Netsys.A.3

Establish NOS Operations Group

\section{Benefits}

This milestone provides reduced administrative overhead while increasing enterprise network security, functionality, and management. 


\title{
Netsys.I.5 Initiate NOS Global Directory
}

\section{Responsible Organization}

IRM

Recommended Initiation Date

$2 \mathrm{Q} / 94$

\section{Sponsoring Organization}

IRM

\section{Status}

Implementation team identified and planning in progress

\section{Anticipated Duration}

12 months

\section{Resource Considerations}

Relatively high-load short-term planning/consulting will become a low-resource operations task.

\section{Discussion}

The NOS directory system is the access mechanism to control enterprise network services, manage user accounts, and authenticate users for file and print services. Some data in this system will be synchronized with information from the site personnel roster and HR databases. In the future, the NOS directory may interface to host services directly or via central network authentication service.

\section{Action Items}

1) Establish group to determine site requirements.

2) Performs NOS upgrade.

3) Migrate remaining services

4) Synchronize connection with HR data.

\section{Dependencies}

\author{
Netsys.l.4 Migrate to New NOS \\ Netsys.A.3 Establish NOS Operations Group
}

\section{Benefits}

This milestone results in a single user ID and login process for all file and print services. By eliminating multiple user accounts, it significantly reduces administrative overhead and improves user functionality. 


\section{Netsys.l.6 Initiate Network Authentication Service}

Responsible Organization

IRM

Recommended Initiation Date

$1 Q / 95$

\section{Anticipated Duration}

24 months

\section{Resource Considerations}

Procurement of DCE-based software interfaces or gateways will vary by platform. It is reasonable to expect host pricing to be significant, while mid-range and small servers might obtain their interfaces by upgrade of the operating system under maintenance. Testing and implementation will require one to two FTEs per platform during the migration process.

\section{Discussion}

Conversion of system-based user authentication to network-based authentication further reduces administrative overhead and increases user functionality. This requires network authentication server(s) and modification of host/server operating systems to support networkbased authentication protocols, technologies which are currently emerging.

Action Item

1) Conduct laboratory-scale testing based on early DCE products.

2) Work with key vendors to attempt to influence DCE development.

3) Install DCE-compliant operating systems as they become available.

4) Interface NOS directory services to DCE.

\section{Dependencies}

Netsys.I.3

Netsys.I.4

Ensure Broad Availability of Networked Workstations

Netsys.I.5

Networks.T.5
Initiate NOS Global Directory

Make DCE Security Services Available in Key Environments

\section{Benefits}

Network authentication provides users with network-transparent computing. That is, a user is able to authenticate him- or herself once and gain access to various network services and data without further authentication. This helps provide the effect that there is a single location for services and data, the network. 


\section{Netsys.I.7 Complete Installation of Territorial File Servers}

\section{Responsible Organization}

IRM

Recommended Initiation Date

$1 Q / 94$

\section{Sponsoring Organization}

IRM

Status

$30 \%$ complete

\section{Anticipated Duration}

30 months

\section{Resource Considerations}

Capital funding for server purchases and/or upgrades is planned under the current MIEs. It will require at least two FTEs to perform the installations.

\section{Discussion}

Installation of an infrastructure-standard file server for each sub-network in a given SRS area or WSRC offsite location will be required to provide the file, print, and application services, as well as the software distribution function.

\section{Action Items}
1) Identify sub-network requirements.
2) Install existing servers.
3) Develop specifications and procure new servers.
4) Install new file servers.

\section{Dependencies}

$\begin{array}{ll}\text { Netsys.A.1 } & \text { Set Site Direction, Funding, and Priority } \\ \text { Netsys.A.3 } & \text { Establish NOS Operations Group } \\ \text { Netsys.I.4 } & \text { Migrate to New NOS } \\ \text { Netsys.I.5 } & \text { Initiate NOS Global Directory }\end{array}$

\section{Benefits}

The territorial file server is the basis for client software distribution and asset management. These servers provide the site-standard applications and private storage space for users. To minimize network traffic, one of these servers must exist in each routed sub-network. Client workstations in the sub-net served must be properly configured to reduce network traffic. 


\section{Netsys.l.8 Manage IP Addresses}

\section{Responsible Organization}

IRM

Recommended Initiation Date

$1 Q / 94$
Sponsoring Organization

IRM

Status

BOOTP IP management program in progress

\section{Anticipated Duration}

Six months

\section{Resource Considerations}

All new workstations must be configured properly at installation; current workstations will require reconfiguration. These are significant but one-time labor-intensive tasks.

\section{Discussion}

All workstations will eventually require an IP address, both for current host access and for future NOS access. A limited number of addresses in each sub-net must be uniquely assigned. Automated processes to assign and maintain these addresses must be developed and implemented to ensure reliability and reduce administrative overhead.

\section{Action Items}

1) Identify sub-network requirements.

2) Install BOOTP servers in each sub-net.

3) Convert workstations to utilize BOOTP process.

4) Establish SRS procedures to manage IP address tables.

\section{Dependencies}

None

\section{Benefits}

A unique IP address is needed to access current IBM and DEC hosts and future Unix application servers. This address must be re-configured when a workstation is physically moved from one sub-net to another or when the sub-net itself is re-configured. Automation of this process reduces support calls from the user community, improves security, assists in asset tracking, and reduces overall administration burden. 


\section{Netsys.I.9 Implement Robust File/Print/Application Services}

Responsible Organization

IRM

Recommended Initiation Date

$3 \mathrm{Q} / 94$

\section{Anticipated Duration}

24 months

\section{Resource Considerations}

Ongoing user-input steering team.

\section{Sponsoring Organization}

IRM

\section{Status}

Not started

\section{Discussion}

An infrastructure system will not be utilized or trusted unless the services provided are more desirable than what is locally available, reliable, and of consistently high quality. Once users can obtain infrastructure services by default, they will embrace those services. This will in turn encourage the use of standards and eliminate some user resistance. It is technically feasible to print from all host and PC-based applications to all LAN and host-attached printers.

\section{Action Items}

1) Continuously review user needs.

2) Establish an automated procedure for obtaining alternate services.

3) Establish consolidated printing program.

4) Encourage site-wide applications.

\section{Dependencies}

\section{Netsys.I.7}

Netsys.I.8

Netsys.P.4

Netsys.T.2
Complete Installation of Territorial File Servers

Manage IP Addresses

Implement Disaster Recovery Procedure

Implement Transparent Print Services on All Platforms

\section{Benefits}

Site licensed or site-developed applications may be distributed and/or maintained more easily on a limited number of application (territorial) servers. The total amount of disk storage necessary is reduced when shared on a server. Peripheral and data sharing will be encouraged. 


\section{Netsys.l.10 Implement Automatic Software Distribution}

Responsible Organization

IRM

Recommended Initiation Date

$1 \mathrm{Q} / 94$

\section{Sponsoring Organization}

IRM

Status

$10 \%$ complete

\section{Anticipated Duration}

36 months

\section{Resource Considerations}

Software purchase or development will not be costly. The policies and procedures needed to implement good software distribution will require at least two FTEs for implementation.

\section{Discussion}

Software installation and upgrade is a highly labor-intensive task. Automated processes will be used to ensure that both servers and clients are maintained at current levels.

\section{Action ltems}

1) Continue to implement network/workstation/NOS infrastructure.

2) Formalize site distribution of personal computer via this infrastructure.

3) Work with vendors to develop concurrent-use licensing for special use software.

\section{Dependencies}

$\begin{array}{ll}\text { Netsys.C.1 } & \text { Implement Standards } \\ \text { Netsys.I.2 } & \text { Upgrade Workstations to Support Netsys Requirements } \\ \text { Netsys.I.7 } & \text { Complete Installation of Territorial File Servers } \\ \text { Netsys.A.2 } & \text { Define Security Principles }\end{array}$

\section{Benefits}

Ensuring that software is automatically at current levels reduces support and administration and will be key to proper utilization of true client/server applications in the future. 


\title{
Netsys.l.11 Connect Office Peripherals Directly to Network
}

\section{Responsible Organization}

IRM

Recommended Initiation Date

$1 \mathrm{Q} / 94$

\section{Sponsoring Organization}

IRM

Status

Started

\section{Anticipated Duration}

36 months

\section{Resource Considerations}

All new peripherals should be purchased network-ready. Where not part of the standard peripheral, the incremental upgrade cost will be small. A one-time installation effort is required for each peripheral. Proper documentation will reduce this effort to a very minimal number. The cost (hardware and labor) of moving existing peripherals must be evaluated in terms of the current value of the existing equipment.

\section{Discussion}

Although hardware costs have generally declined, there is an increasing need and desire for higher quality peripherals. Proper sharing of enhanced peripherals satisfies this need while still reducing overall costs.

\section{Action ltems}

1) Document guidelines for procurement of shared peripherals.

2) Migrate current peripherals to direct network connection.

3) Facilitate use of shared peripherals with better user interfaces.

\section{Dependencies}

\author{
Netsys.A.1 Set Site Direction, Funding, and Priority \\ Netsys.I.3 Ensure Broad Availability of Networked Workstations \\ Netsys.I.9 Implement Robust File/Print/Application Services \\ Netsys.C.1 Implement Standards \\ Netsys.C.2 Share Equipment/Peripherals
}

\section{Benefits}

This milestone encourages cost-effective utilization of network resources and provides increased user functionality. 


\section{Netsys.l.12 Implement Reliable Performance Monitoring}

Responsible Organization

IRM

Recommended Initiation Date

$3 Q / 94$

\section{Sponsoring Organization}

IRM

Status

Not started

\section{Anticipated Duration}

12 months

\section{Resource Considerations}

Most of the required software has already been procured. Implementation will require about six months of an FTE. Once placed into operation, one FTE should accommodate the monitoring and tuning functions.

\section{Discussion}

Consistent, reliable network services to the user can only be guaranteed if the infrastructure as a whole is monitored for performance and failure alerts. Proactive monitoring can also help tune the system for better response and equipment utilization. Additions of monitoring software at the client workstation will allow performance monitoring of both network protocols and file/print/application services.

\section{Action Items}

1) Implement basic network services management.

2) Re-organize central group responsibilities.

3) Install network management components on workstations (SNMP).

\section{Dependencies}

Netsys.I.3

Netsys.A.3

Ensure Broad Availability of Networked Workstations

Establish NOS Operations Group

\section{Benefits}

Proper monitoring will reduce the possibility of failure, improve overall up-time, and create a trusted system. Automated procedures will reduce central staff workload. 


\section{Network Systems Administrative Milestones}

The following administrative milestones must be resolved to adopt the technology. Each is described in more detail in the following sections.

Netsys.A.1

Netsys.A.2

Netsys.A.3
Set Site Direction, Funding, and Priority

Define Security Principles

Establish NOS Operations Group 


\section{Netsys.A.1 Set Site Direction, Funding, and Priority}

Responsible Organization

IRM

Recommended Initiation Date

$1 Q / 94$
Sponsoring Organization

IRM

\section{Status}

Management acceptance of WSRC Strategic Plan for Business Computing provides focus for this milestone.

\section{Anticipated Duration}

48 months

\section{Resource Considerations}

Computer hardware procurement will be a significant expense. Direction and funding will be part of the strategic planning process.

\section{Discussion}

Establishment of site policy and practices to support central direction, funding, and priority for site information technology (I/T) initiatives.

\section{Action ltems}

1) Activate SRS Computing Council to establish site $I / T$ priorities.

2) Endorse site Strategic Plan for $1 / T$.

3) Develop IRM cost estimates and timing required for site network initiatives for Computer Council approval.

\section{Dependencies}

None

\section{Benefits}

Completion of the site network is a significant site infrastructure issue. Current and future mission assignments require a robust and reliable network that is uniformly implemented across SRS. Without central direction and funding, network implementations tend to be uneven, as certain departments or facilities see the need to fund the network and others do not. 


\section{Netsys.A.2 Define Security Principles}

\section{Responsible Organization}

IRM

Recommended Initiation Date

$2 \mathrm{Q} / 94$

\section{Sponsoring Organization}

IRM

\section{Status}

Not started

\section{Anticipated Duration}

12 months

\section{Resource Considerations}

This will require about three FTEs over the six-month duration. There are no hardware or software costs.

\section{Discussion}

Workgroup computing assumes a posture that the owner of the data designates who should be allowed what level of access. A fully stratified security model will provide various levels of administration such that the data owner can take stewardship. The purpose of this milestone is to envision and document the security model to which the technology should strive.

\section{Action ltems}

1) Assemble user, administrator, and security audit needs.

2) Construct a security model.

3) Review applicable DOE orders.

4) Publish policy, procedure, and principles documentation.

\section{Dependencies}

None

\section{Benefits}

By creating and publishing a desired security architecture, all associated groups will gain a clear understanding of the total needs and restrictions. At each point in the implementation, the technology and procedures may be compared to the model and verified. Although it might not be possible to meet all needs at the outset, this plan provides the guidance necessary to move toward the final goal. 


\section{Netsys.A.3 Establish NOS Operations Group}

Responsible Organization

IRM

Recommended Initiation Date

$2 \mathrm{Q} / 94$

Anticipated Duration

12 months

\section{Resource Considerations}

Once established, this is an ongoing operation which is likely to require two FTEs.

\section{Discussion}

The NOS is an infrastructure upon which networked applications and services are layered. Centralization of the NOS operations is more cost-effective, provides a process for easy upgrade, and ensures a uniform implementation. Establishment of site policy and practices, daily operations, and disaster recovery would be conducted by this group.

\section{Action ltems}
1) Establish centralized IRM group.
2) Conduct internal training.

\section{Dependencies}

None

\section{Benefits}

Increased standardization, reduced support costs, uniform infrastructure. 


\section{Network Systems Cultural Milestones}

The following site culture milestones must be resolved to adopt the technology. Each milestone is discussed in more detail in the following sections.

Netsys.C.1 Implement Standards

Netsys.C.2 Share Equipment/Peripherals

Netsys.C.3 Save Data to Network 


\section{Netsys.C.1 Implement Standards}

Responsible Organization

IRM

Recommended Initiation Date

$2 Q / 94$

\section{Anticipated Duration}

24 months

\section{Resource Considerations}

There are no hardware or software costs. A level of marketing may be required to sample the needs of the user community and communicate information to them.

\section{Discussion}

In the past, standard products were established by CAST, and their applicability was documented. A similar process must be created. Where possible (and cost-effective), site licenses are established as part of the standards-setting process so that users are not required to track individual licenses. Once a site standard product is established, it can be installed on file servers on the site network. Users can use the software directly from the file servers so that end users no longer need to devote their time to software product installation. Likewise, installation of new software version updates can be automated to save end-user time. Management should discourage the use of older, non-standard products and encourage a continuous migration which keeps current with the use of the defined standards.

\section{Action ltems}

1) Define IRM/T\&PC standards-setting process.

2) Document standards.

3) Issue management directives to encourage use of standards.

\section{Dependencies}

None

\section{Benefits}

Use of IT standards provides improved interoperability of SRS systems and applications, as well as lower overall costs. 


\section{Netsys.C.2 Share Equipment/Peripherals}

\section{Responsible Organization}

IRM

Recommended Initiation Date

$1 Q / 94$

\section{Sponsoring Organization}

IRM

Status

$10 \%$ complete

\section{Anticipated Duration}

36 months

\section{Resource Considerations}

Shared resources required will be less than the current practices.

\section{Discussion}

Future technologies will allow greater personalization of shared equipment. There must be a user move away from the notion that the workstation or printer is personal property. Moving personalization and data to the enterprise network and providing more robust infrastructure services will aid the required management directives.

Action Items

1) Complete the network infrastructure.

2) Factor greater user personalization into the enterprise system.

3) Provide better services on the network than can be locally obtained.

4) Encourage management to direct users toward this goal.

\section{Dependencies}

Netsys.I.3

Netsys.I.7

Netsys.I.9

Netsys.I.11

Netsys.C.1

Netsys.C.3

Netsys.P.4
Ensure Broad Availability of Networked Workstations

Complete Installation of Territorial File Servers Implement Robust File/Print/Application Services Connect Office Peripherals Directly to Network Implement Standards Save Data to Network Implement Disaster Recovery Procedure

\section{Benefits}

Once embraced by the user community, sharing networked resources will provide large cost savings, will improve data sharing and productivity, and will improve security and reliability. 


\section{Netsys.C.3 Save Data to Network}

Responsible Organization

IRM

Recommended Initiation Date

$1 \mathrm{Q} / 94$
Sponsoring Organization

IRM

\section{Status}

$10 \%$ complete

\section{Anticipated Duration}

36 months

\section{Resource Considerations}

This milestone requires completion of the infrastructure and assumes a large amount of reliable, available disk space. Capital funding might be the limiting factor.

\section{Discussion}

Users must feel that data saved to the enterprise network is more reliable and available than that which might be saved locally. By developing security plans which encourage placing information within the network, by demonstrating the values of shared data, and through some management directive concerning data management there will be a shift in process.

\section{Action Items}

1) Complete infrastructure.

2) Review disk space considerations (on-going).

3) User education/training on policies and procedures.

\section{Dependencies}

Netsys.I.3

Ensure Broad Availability of Networked Workstations

Netsys.I.7

Complete Installation of Territorial File Servers

Netsys.I.9

Implement Robust File/Print/Application Services

Netsys.I.11

Office Peripherals with Direct Network Connection

Netsys.C.1

Implement Standards

Netsys.P.4

Implement Disaster Recovery Procedure

\section{Benefits}

The basis for enterprise workgroup data-sharing is the availability of data from any location. Both personal and workgroup data will be more reliably available if saved to the network. 


\section{Network Systems Process Milestones}

The following work-flow milestones must be resolved to adopt the technology. Each is described in detail in the following sections.

Netsys.P.1

Netsys.P.2

Netsys.P.3

Netsys.P.4

Netsys.P.5
Implement Single User Login to Network

Implement Distributed Workgroup Management

Implement Software License Management

Implement Disaster Recovery Procedure

Implement Infrastructure Enhancement Process 


\section{Netsys.P.1 Implement Single User Login to Network}

\section{Responsible Organization}

IRM

\section{Recommended Initiation Date}

$3 \mathrm{Q} / 94$
Sponsoring Organization

IRM

Status

Planning stage

\section{Anticipated Duration}

24 months

\section{Resource Considerations}

Hardware and software costs are accounted for in milestone Netsys.I.6. Future systems should be designed to utilize the available authentication on a routine basis. Internal training of system designers might be necessary.

\section{Discussion}

This milestone involves developing process changes to establish an environment where users log in once to the network. Subsequent use of SRS host computers, applications, or databases is provided without additional user login requirements, taking advantage of networked-based authentication technology.

\section{Action ltems}

1) Establish user login procedures.

2) Implement security gateways where possible.

3) Migrate systems to DCE.

\section{Dependencies}

Netsys.I.3

Netsys.I.5

Netsys.I.6

Networks.T.5
Ensure Broad Availability of Networked Workstations

Initiate NOS Global Directory

Initiate Network Authentication Service

Make DCE Security Services Available in Key Environments

\section{Benefits}

Network authentication provides users with network-transparent computing. That is, a user is able to authenticate him- or herself once and gain access to various network services and data without further authentication. This helps provide the effect that there is a single location for services and data, the network. 


\section{Netsys.P.2 Implement Distributed Workgroup Management}

Responsible Organization

IRM

Recommended Initiation Date

$3 \mathrm{Q} / 94$
Sponsoring Organization

IRM

\section{Status}

Not started

\section{Anticipated Duration}

36 months

\section{Resource Considerations}

No commercial hardware or software products are required. Some locally developed user interface tools might be utilized. Formulation of policies and procedures, internal systems planning/training, and user training will be required.

\section{Discussion}

This milestone moves the control of data access to the owner of the data and removes centralized administrative burden.

\section{Action Items}

1) Define policies and procedures.

2) Implement central administration of overall disk space.

3) Conduct user education.

\section{Dependencies}

Netsys.I.5

Initiate NOS Global Directory

Netsys.I.6

Initiate Network Authentication Service

Netsys.I.7

Complete Installation of Territorial File Servers

Netsys.A.3

Establish NOS Operations Group

\section{Benefits}

The movement of access control reduces central administration and improves data availability. 


\section{Netsys.P.3 Implement Software License Management}

\section{Responsible Organization}

IRM

Recommended Initiation Date

$4 \mathrm{Q} / 94$

\section{Sponsoring Organization}

IRM

\section{Anticipated Duration}

30 months

\section{Resource Considerations}

Procurement of additional software to work with the NOS will be required. In some cases this may be furnished as part of other purchased software. Effort to install and maintain the system should be minor.

\section{Discussion}

Widely used or site-standard software must be available to various workstations on an asneeded basis depending on user needs. Various license schemes which include site-agreement, concurrent-use, or specific machine or specific individual must be accommodated. Although market direction appears directed toward technologies which might address these needs (network license service), there must also be business policy regarding license administration.

\section{Action Items}

1) Continue to track market trends in software licensing.

2) Consider license administration in determining software costs.

3) Establish business policy on availability of software to individuals.

\section{Dependencies}

Netsys.1.3

Netsys.I.7

Netsys.I.10

Netsys.A.2

Netsys.C.1
Ensure Broad Availability of Networked Workstations

Complete Installation of Territorial File Servers

Automatic Software Distribution

Define Security Principles

Implement Standards

\section{Benefits}

It is imperative to provide policy, procedure, and implementation which provide a cost-effective way of ensuring that software use is legal and best suited to user requirements. 


\section{Netsys.P.4 Implement Disaster Recovery Procedure}

Responsible Organization

IRM

Recommended Initiation Date

$3 Q / 94$
Sponsoring Organization

IRM

\section{Status}

Not started

\section{Anticipated Duration}

12 months

\section{Resource Considerations}

This will require some capital for hot-spare equipment, and some short-term planning and testing efforts.

\section{Discussion}

A fully tested plan must be in place to replicate hardware and/or data in the event of a disaster. This plan should include simple loss of a single file server to an event such as a major building fire which destroyed the telecommunications and file server areas. The plan should document level of cost associated with each level of risk.

\section{Action ltems}

1) Plan and procure hot spare equipment.

2) Provide reliable backup services and offsite storage.

3) Plan and test recovery procedures.

4) Document policies and procedures and level of acceptable risk.

\section{Dependencies}

Netsys.A.3 Establish NOS Operations Group

\section{Benefits}

Mission-critical data cannot be trusted to a system until the level of risk has been assessed and recovery plans established. 


\section{Netsys.P.5 Implement Infrastructure Enhancement Process}

Responsible Organization

IRM

Recommended Initiation Date

$2 \mathrm{Q} / 94$
Sponsoring Organization

IRM

Status

Not started

\section{Anticipated Duration}

24 months

\section{Resource Considerations}

After an initial investment in creating and documenting the process, this will require only a small ongoing effort. Major changes in user needs or in the technologies implemented will cause resource needs to peak.

\section{Discussion}

A process must be established to allow planning input from users, developers, and technologists so that the ongoing networked services migrate as needs and solutions change. This process would establish priorities, provide change control, and ensure compatibility.

\section{Action ltems}

1) Establish steering team.

2) Document current environment.

3) Document input and change-control process.

4) Provide for regular meetings/input from the team members.

\section{Dependencies}

None

\section{Benefits}

This will ensure that the enterprise system best meets the user needs in a timely fashion. 


\section{Network Systems Technology Milestones}

The following technology milestones must be resolved to adopt the technology. Each is described in more detail in the following sections.

Netsys.T.1 Deliver NOS over TCP / IP

Netsys.T.2 Implement Transparent Print Services on All Platforms 


\section{Netsys.T.1 Deliver NOS over TCP/IP}

Responsible Organization

IRM

Recommended Initiation Date

$1 \mathrm{Q} / 95$

Anticipated Duration

12 months

\section{Resource Considerations}

This is likely to be included in the maintenance of the current standard NOS.

\section{Discussion}

This will move NOS services to a single networking protocol (TCP/IP) more closely in line with other enterprise network services and hosts.

\section{Action ltems}

1) Continue to communicate with NOS vendor.

2) Test emerging technologies.

3) Finalize IP address management.

4) Test future NOS products as early as possible.

\section{Dependencies}

Netsys.I.4

Migrate to New NOS

Netsys.I.8

\section{Benefits}

A single protocol for all networked services (NOS and hosts) provides improved performance and reduced administration. 


\section{Netsys.T.2 Implement Transparent Print Services on All Platforms}

Responsible Organization

IRM

Recommended Initiation Date

$4 \mathrm{Q} / 94$

\section{Anticipated Duration}

12 months

\section{Resource Considerations}

Might require some hardware/software purchases in the form of gateways or transfer processes. Development of the vision and first implementation might require several FTEs, but ongoing operations should require less than the current level of effort.

\section{Discussion}

Printing should be a network service, available equally to all platforms. All printing should occur at the most convenient and cost effective location for the user. The process for printing should be generalized across LAN and host services so as to appear to be the same to the user.

\section{Action ltems}

1) Document printing needs for each platform.

2) Determine user operation profiles.

3) Investigate printing alternatives.

4) Plan user training.

\section{Dependencies}

Netsys.I.3

Netsys.A.2

Netsys.A.3
Ensure Broad Availability of Networked Workstations

Define Security Principles

Establish NOS Operations Group

\section{Benefits}

The implementation of an enterprise-wide approach to printing would better utilize shared printer equipment, improve user productivity, and reduce central administration costs. 


\section{Network Systems Miscellaneous Milestones}

The following additional milestones, not covered by any other issue area, must be resolved to adopt the technology. Each of these is described in more detail in the sections below.

Netsys.M.1 Implement PC Utilization Plan 


\section{Netsys.M.1 Implement PC Utilization Plan}

Responsible Organization

IRM

Recommended Initiation Date

$2 \mathrm{Q} / 94$
Sponsoring Organization

IRM

\section{Status}

In progress as part of WSRC Strategic Plan for Business Computing

\section{Anticipated Duration}

12 months; ongoing

\section{Resource Considerations}

There is an implication of available capital to purchase computers based on a recommendation from this planning process. The actual planning will require cross-division coordination.

\section{Discussion}

Workstations are the key to networked systems implementation. Purchase and re-distribution of equipment based on user needs and priorities will ensure that minimum equipment needs for enterprise applications will be met.

\section{Action Items}

1) Establish site policy on minimum $P C$ requirements for network attachment.

2) Establish obsolete PC retirement policy.

\section{Dependencies}

Netsys.A.1

Set Site Direction, Funding, and Priority

\section{Benefits}

Effective management of user personal computers depends on attaching these system in a WISDOM-like environment. Such an environment provides for network monitoring of workstations as well as automatic configuration and software updates over the network. Obsolete systems are unable to participate and thus are unmanageable except by manpowerintensive efforts. 


\section{Information Warehouse}

\section{Introduction and Destination}

As stated in the SRS Computing Architecture, the future vision of computing at the Savannah River Site is a "standards-based, data-driven, and workstation-oriented computing environment." An information warehouse (IW) for end-user query and management information reporting is one means to achieve this vision. An information warehouse establishes a managed information environment where data, specific to the management of an enterprise (for example, WSRC), is extracted from the data collections or operational environment and migrated to an integrated reporting environment that is specifically tuned to query and end-user access. The IW will be managed by an information repository and supported by a standard suite of query/access tools. Data usage will be carefully monitored and controlled through audits, data policies, and procedures.

The IW becomes the standard source for management reporting, replacing the free market reporting and query environment described in the following section.

The principle objective for implementing an IW at WSRC is to substantially relieve mainframe capacity constraints and reduce the standing applications backlog. An IW will shift the reporting of management information away from the processes that collect the data (for example, data collections systems and applications) to the end-user community, the principal user of the data. The WSRC Strategic Plan for Business Computing identified four areas for IW support. These are:

1. People data to address site need for reliable and readily accessible person and organization data.

2. Financial data to directly address immediate customer needs.

3. Assets and Facilities data to provide a common point of integration of asset data gathered from financial, property management, and operational sources.

4. Purchasing data to relieve the significant Procurement Cycle System (PCS) backlog for reporting and eliminate redundant local databases used to track commitments.

The SRS information warehouse will consists of four components:

1. Information Repository where the meta-information (that is, information about information) is defined and accessible to all users.

2. Data Extracts which control the extraction of warehouse data from the data collection systems.

3. Data Standardization where data is integrated by subject or functional area, and summarized or otherwise transformed according to predefined rules and data management standards and policies.

4. Data Stores organized by subject or functional area.

a. Global warehouse which contains the management information supporting the business or management requirements of SRS. 
b. Local (or remote) warehouse which contains subsets of the global warehouse, specifically tuned for individual department or group level.

c. Archival warehouse which contains inactive data.

Several publications are available, including the SRS Application Design Architecture (WSRCIM-92-109), to explain the concepts of IW technology and the separation of management data from operational data.

\section{Starting Point}

Pockets of information warehouse activities exist today at SRS. IW type activities are found within application- or department-specific reporting, numerous data extracts and downloads, and individual what-if databases. Several business systems currently offer or plan to offer reporting databases. IW type activities are also supported by numerous query tools, workstation-oriented data products, and individual efforts to integrate data. These efforts are without overall management and direction, and have caused numerous data conflicts and misunderstandings.

In a typical IS development group, information reporting accounts for the majority of the maintenance backlog and development efforts. This may be true for SRS's application development organizations. This backlog will not diminish unless there is a fundamental change in the reporting of management information. Implementing an IW will fundamentally change the nature of reporting by transferring the responsibility for reporting away from the processes or systems which collect data to the end users responsible for its reporting, analysis, and content.

The following section contains a series of milestones which identify the events necessary for the successfully transition from the current state (starting point) of information reporting to the IW environment (destination). 


\section{Information Warehouse Infrastructure Milestones}

The following infrastructure milestones must be resolved to adopt the technology. Each is described in more detail in the following sections.

IW.I.1

Establish a Communications Network Capability Supporting the Global and Local Warehouses

IW.I.2

Establish Client SQL-Network

IW.I.3

Establish/Populate IW Component of Data Repository

IW.I.4

Identify and Procure IW Support Tools and Hardware

IW.I.5

Identify and Procure Archival Management Tools and Hardware 


\section{IW.I.1 Establish a Communications Network Capability Supporting the Global and Local Warehouses}

Responsible Organization

IRM

Recommend Initiation Date

$1 Q / 94$
Sponsoring Organization

IRM

Status

In process

\section{Anticipated Duration}

48 months

\section{Resource Considerations}

See Networked Systems Roadmap.

\section{Discussion}

Success of IW program is dependent upon the rapid movement of data between/among platforms, DBMS, and workstations. The communications network must be capable of supporting the transfer of operational data to the global IW, supporting end-user data queries and data transfers, and supporting transfer of global IW data to local servers. The network requirements (that is, size and type) can be determined in part through the IW Model (IW.P.2). However, the load on the network attributed to IW will be highly variable, subject to the number of simultaneous users, type of queries, number of data transfers, and other related factors.

\section{Action Items}

1) Identify potential impact of IW on existing network.

2) Define IW network requirements.

\section{Dependencies}

\section{IW.P.2}

Netsys.I.3

Netsys.1.7

Networks.I.5
Define the IW Model

Ensure Broad Availability of Networked Workstations

Complete Installation of Territorial File Servers

Install Ethernet/FDDI Network Trunks

\section{Benefits}

See Roadmap for Networked Systems. 


\section{IW.I.2 Establish Client SQL-Network}

Responsible Organization

IRM

Recommend Initiation Date

$2 \mathrm{Q} / 94$
Sponsoring Organization

IRM

\section{Status}

Initial efforts under way; SQL*net identified for addition to WISDOM

\section{Anticipated Duration}

24 months with the majority of the effort in the first year

\section{Resource Considerations}

In addition to the resources of the IW implementation team, this milestone requires the support of several IRM organizations.

\section{Discussion}

A client SQL connection may be required by the IW RDBMS. For example, the Oracle RDBMS requires a SQL connection (SQL*net) on both the server and client; other RDBMS may require the same. One of the major issues identified by the IW pilot project was the inability of team members to connect with the pilot warehouse via several different query tools due to differences in the SQL installed on the site's various workstations and local area networks.

\section{Action ltems}

1) Identify client requirements for SQL connection to IW.

2) Procure standard client SQL connection product.

3) Identify software change management technique for managing distribution of the client SQL connection.

4) Install client SQL connection on WISDOM workstations.

\section{Dependencies}

\section{IW.I.4}

IW.A.1

TW.P.2

IW.P.5

Netsys.I.3

Netsys.I.7

Networks.I.5
Identify and Procure IW Support Tools and Hardware

Establish IW Implementation Team

Define the IW Model

Establish Local or Remote IWs

Ensure Broad Availability of Networked Workstations

Complete Installation of Territorial File Servers

Install Ethernet/FDDI Network Trunks

\section{Benefits}

Allows SQL end-user tools to connect with the Oracle data warehouses. 


\section{IW.I.3 Establish/Populate IW Component of Data Repository}

Responsible Organization

IRM

Recommend Initiation Date

$1 Q / 94$

\section{Anticipated Duration}

36 months for initial effort

\section{Resource Considerations}

This milestone requires the resources of several IRM functions. The principal resource is the data management function, which has the responsibility for overall data management, and the IW implementation team, which has the IW metadata responsibilities. Additionally, the resources of several application programmers is necessary to automate the transfer of data definitions from existing catalog systems to the repository.

\section{Discussion}

Data is created, maintained, and utilized by a variety of automated systems, individuals, and organizations. Additionally, information necessary to the safe, cost-effective, and efficient operation and management of SRS is contained in a variety of DBMSs, file cabinets, and desk drawers. Knowledge that the data exists, its source, rules or constraints, and its definitions or attributes must be defined to and managed by a (logically) central data repository. The IW repository is an extension of the central repository. The metadata requirements for warehouse data are slightly different than those for a normal repository. A partial list of these requirements would include the rules for data extraction, rules for data transformation, extract job, system of record or source for the data, access restrictions, date of the most recent extraction, and information regarding archival requirements.

The rationale for a repository is discussed in the SRS Applications Design Architecture.

\section{Action ltems}

1) Identify IW repository requirements.

2) Procure IW repository

3) Establish policies and procedures for IW repository.

4) Populate the repository with metadata from the IW logical model

\section{Dependencies}
IW.P.2
Define the IW Model
IW.I.4 Identify and Procure IW Support Tools and Hardware

\section{Benefits}

Effective utilitzation of an IW is facilitated by an IW repository. The repository documents the contents of the warehouse and provides a definitive or single source for the user community to 
access when researching the availability of data, definition of data, or data attributes. The repository as a knowledge holder of meta-information identifies for each data item in the warehouse its system of record, custodian, business rules, and extract schedule and job, plus a host of other meta information. 


\section{IW.I.4 Identify and Procure IW Support Tools and Hardware}

\section{Responsible Organization}

IRM

Recommend Initiation Date

$1 \mathrm{Q} / 94$

\section{Sponsoring Organization}

IRM

\section{Status}

Initial planning under way

\section{Anticipated Duration}

18 months

\section{Resource Considerations}

The IRM IW pilot project identified the prime tool suite (that is, repository manager, subscription service, query tools, DBMS, communications software, SQL, server/s, and storage hardware) required to support the global and summary warehouse. In addition to the core IW team, this milestone requires support from IRM organizations to define the requirements for end-user query tools and to install the software and hardware. Support is required from ALD's site procurement organization.

The Information Warehouse(s) will require dedicated platform(s) requiring substantial capital (hardware) and software costs. These costs are being factored in to the WSRC Strategic Plan for Business Computing.

\section{Discussion}

The WSRC Strategic Plan for Business Computing states that "Development of enterprise data information warehouse environment requires major computational resources to support large amounts of financial, personnel, asset, and environmental data." The thrust of this milestone is the procurement and installation of the tools and hardware to support the IW. Its the responsibility of the IW implementation team, working in conjunction with supporting IRM organizations, to define the requirements for the DBMS engine, query and reporting tools, repository, and storage devices.

\section{Action ltems}

1) Procure IW hardware as identified in IW.P.2.

2) Define, procure, and install IW tool suite.

\section{Dependencies}

IW.P.2

Define the IW Model

IW.A.1

Establish IW Implementation Team

\section{Benefits .}

A standardize/integrated tool suite (for example, query, IW management, repository, and data dictionary services) offers several advantages to the computing professional and end-user community. Fewer products to learn, interoperability between components of the technology, and reduced risk of data failures are several of the potential benefits associated with tools. 


\section{IW.I.5 Identify and Procure Archival Management Tools and Hardware}

Responsible Organization

IRM

Recommend Initiation Date

$1 \mathrm{Q} / 95$

\section{Sponsoring Organization}

IRM

\section{Status}

Not started

\section{Anticipated Duration}

12 months

\section{Resource Considerations}

Manpower from ALD's PMMD and IRM organizations is required to support the procurement, installation, and testing of the IW software and hardware.

\section{Discussion}

As stated in the discussion of IW.P.6, archival management of an Information Warehouse is not well understood; therefore, the suite of tools necessary to support this warehouse has not been developed. However, as the requirements for archiving, retrieval, and repository management are identified, the IW software vendors will begin to develop products. It is anticipated that vendors will begin to develop products in the second half of 1994 or early in 1995.

\section{Action ltems}

1) Procure archival storage hardware.

2) Procure archival management software.

\section{Dependencies}

None

\section{Benefits}

The archiving of non-active IW data with the ability to recall the data on demand has tremendous benefits to an IW. Some of the more obvious benefits are reduced online storage requirements or improved query response time due to smaller number of indexes and active data. However, archival management provides an additional benefit: long-term management of critical information. Features of this management would include tracking the location of the data with an ability to recall the data to active storage upon demand. 


\section{Information Warehouse Administrative Milestones}

The following administrative milestones must be resolved to adopt the technology. Each is described in more detail in the following sections.

IW.A.1

IW.A.2

Establish IW Implementation Team

IW.A.3

Establish Long-Term Support Organization

IW.A.4

Establish IW Polices and Procedures

Replace Redundant Operational Reports, Screens, and Data Feeds 


\section{IW.A.1 Establish IW Implementation Team}

Responsible Organization

IRM Management

Recommend Initiation Date

$1 Q / 94$

\section{Sponsoring Organization}

IRM

\section{Status}

IW implementation teams have been initiated as part of the WSRC Strategic Plan for Business Computing.

\section{Anticipated Duration}

Six months, or until succeeded by permanent IW support organization.

\section{Resource Considerations}

The core team should consist of three to four members with multiple skills. Some examples are data management, application development, database management, and business analysis. However, the team will require augmentation by several IRM and SRS organizations.

\section{Discussion}

Implementing an IW requires the knowledge, talents, and services of several organizations, knowledge users, and computer information specialists. Appointing a cross-sectional, multitalented team to manage the implementation is necessary to the success of the IW implementation and the transition to an IW environment.

\section{Action ltems}

1) Establish implementation team(s) and appoint team leader.

2) Select personnel.

3) Transfer selected personnel into group.

4) Conduct intensive IW training for group members.

\section{Dependencies}

None

\section{Benefits}

Each team is chartered and empowered to implement a production IW. 


\title{
IW.A.2 Establish Long-term Support Organization
}

Responsible Organization

IRM Management

Recommend Initiation Date

$3 Q / 94$

\section{Sponsoring Organization}

IRM

\section{Anticipated Duration}

Three months

\section{Resource Considerations}

A support organization consisting of two or more members would facilitate the transition to IW by providing long-term continuity, consistency in IW structure, and standardization of the process.

\section{Discussion}

Continuity and long-term management of an IW can best be served by a dedicated support organization whose charter is the day-to-day management of the warehouse. Ideally, the group would consist of persons skilled in data management, application development, database management, and business analysis. The group would provide the management oversight necessary to prevent the creation of isolated warehouses, coordinate warehouse development efforts, and manage the warehouse environment. This group is the successor to the $I W$ implementation team.

\section{Action Items}

1) Select and transfer personnel to IW support group

2) Train organization members in $I W$ concepts and principles (as required).

\section{Dependencies}

IW.A.1

\author{
Establish IW Implementation Team
}

\section{Benefits}

Establishing a formal support organization ensures the continuation of the work begun by the IW implementation team and provides the structure for successful transition to IW technology. 


\title{
IW.A.3 Establish IW Polices and Procedures
}

Responsible Organization

IRM

Recommend Initiation Date

$2 \mathrm{Q} / 94$

\section{Sponsoring Organization}

IRM

Status

Not started

\section{Anticipated Duration}

Six months

\section{Resource Considerations}

Resources from several IRM organizations are required to support this milestone. Data resource has the lead responsibility for this milestone, augmented by the IW implementation team, application development methodology specialists, and application development organizations.

\section{Discussion}

Transition to an IW environment (as the standard management reporting vehicle) cannot be successful until technology is incorporated into the culture of the application development community.

\section{Action ltems}

1) Establish policies and procedures for IW population, content, usage, data standards, and access.

2) Incorporate IW technology into IRM System Development Methodology Manual.

\section{Dependencies}

\author{
IW.A.1 \\ Establish IW Implementation Team \\ IW.A.2 \\ Establish Long-term Support Organization
}

\section{Benefits}

Incorporating IW into the IRM System Development Methodology Manual and establishing IW data policies and procedures will facilitate the transition to IW and help ensure the success of the warehouse. 


\section{IW.A.4 Replace Redundant Operational Reports, Screens, and Data Feeds}

Responsible Organization

IRM

Recommend Initiation Date

$4 Q / 94$

\section{Anticipated Duration}

18 months

\author{
Sponsoring Organization
}

IRM

\section{Status}

Not started

\section{Resource Considerations}

This milestone requires participation by IRM application development organizations with support from the IW support organization.

\section{Discussion}

A successful IW program has the potential to eliminate redundant data feeds, management reports, and online query screens. However, experience has proven that once a report or screen is created and ingrained into the culture, it simply never goes away. The intent of this milestone is to establish an on-going program to identify redundancies and schedule their replacement by the IW.

\section{Action Items}

1) Identify major data feeds, reports, and online transaction/query screens which can be supported via the warehouse.

2) Create migration strategy whose end goal is the elimination of redundant data feeds, reports, and online query screens.

\section{Dependencies}

IW.C.1

Establish Training for Application-development Community

IW.C.2

Establish Training for End-User Community

IW.A.2 Establish Long-term Support Organization

IW.P.2

Define the IW Model

\section{Benefits}

Eliminating redundant reports and online transaction/query screens has several benefits for IRM; for example, reduction of the number of screens or reports that must be maintained, and reduction of the resources which generate or otherwise support the redundancy. 


\section{Information Warehouse Cultural Milestones}

The following cultural milestones must be resolved to adopt the technology. Each milestone is discussed in more detail in the following sections.

IW.C.1 Establish Training for Application-Development Community

iW.C.2 Establish Training for End-User Community 
IW.C.1 Establish Training for Application-development

\section{Community}

\section{Responsible Organization}

Training Integration

Recommend Initiation Date

$2 Q / 94$

\section{Sponsoring Organization}

IRM/IW

Status

Not started

\section{Anticipated Duration}

Nine months

\section{Resource Considerations}

Resources from several organizations (for example, Training Integration, ALD's PMMD, and IRM) are necessary to support this milestone. The core resources are the IW implementation team (IW.A.1), the IW support organization (IW.A.2), and Training Integration Department, augmented by industry experts .

\section{Discussion}

To successfully transition from our current environment to an IW environment will require a significant change in the site's approach to information reporting and appreciation of data utility (for example, that data is a resource to be shared with all who have a valid need). Training the application development community in the concepts of the IW technology is a necessary prerequisite for successful implementation of the technology. The training provides a foundation for SRS application developers to understand the role of data, the role of the enduser community in information reporting, and the concepts of data separation (management or decision support data from operational data). Training will enable application developers to build solutions that support the warehouse approach.

\section{Action ltems}

1) Develop training plan and schedule with Training Integration (TI).

2) Develop training guide.

3) Train $\mathrm{TI}$ instructors.

4) Implement training program.

5) Review and modify plan and guide as necessary.

\section{Dependencies}

IW.A.1 - Establish IW Implementation Team

IW.A.3 Establish IW Policies and Procedures

IW.I.3 Establish/Populate IW Component of Data Repository

IW.I.4 Identify and Procure IW Support Tools and Hardware 


\section{Benefits}

The successful transition to an IW environment can be facilitated when the user community (application development and end user) understands the concepts, limitations, and potentials of the technology. 


\section{IW.C.2 Establish Training for End-User Community}

Responsible Organization

Training Integration

Recommend Initiation Date

$4 \mathrm{Q} / 94$
Sponsoring Organization

IRM

Stấus

Not started

\section{Anticipated Duration}

24 months

\section{Resource Considerations}

This milestone requires support from several organizations (for example, Training Integration, Procurement, and IRM) and touches many WSRC organizations. The core resources are the IW implementation team (IW.A.1), the IW support organization (IW.A.2) supplemented by Training Integration and industry experts.

\section{Discussion}

Transition to an IW environment places new demands on the SRS end-user community as the responsibility for management information reporting shifts from the application development community to the end-user community. Training is a necessary prerequisite for successful transition and end-user success with the Information Warehouse.

The areas in which the end-user community should receive training are the concepts of IW technology, techniques for accessing IW data, and use of the IW tool suite. Additionally, the training should review the policies and procedures governing usage of IW data.

\section{Action Items}

1) Develop training program with Training Integration.

2) Develop end-user training guide.

3) Identify and train instructors.

4) Initiate training program.

5) Review results and modify program as necessary.

\section{Dependencies}

IW.A.3

IW.I.3

IW.I.4
Establish IW Policies and Procedures

Establish/Populate IW Component of Data Repository

Identify and Procure IW Support Tools and Hardware

\section{Benefits}

The value of an IW is directly proportional to the ability of its users to competently and comfortably access the warehouse data. Through a continuing program of education, prospective users would become knowledgeable in the contents of the warehouse, ways to efficiently and effectively access its data, and the capabilities and use of in the IW tool suite. 


\section{Information Warehouse Process Milestones}

The following work flow milestones must be resolved to adopt the technology. Each is described in detail in the following sections.

[W.P.1

IW.P.2

IW.P.3

IW.P.4

IW.P.5

IW.P.6
Identify and Prioritize Business Requirements for Global and Local Warehouses

Define the IW Model

Implement the Global Warehouse

Expand Global Warehouse

Establish Local or Remote IWs

Establish Archival IW 


\section{IW.P.1 Identify and Prioritize Business Requirements for Global and Local Warehouses}

Responsible Organization

IRM

Recommend Initiation Date

$1 Q / 94$
Sponsoring Organization

IRM

Status

In progress as part of WSRC Strategic Plan for Business Computing

\section{Anticipated Duration}

Six months

\section{Resource Considerations}

IW implementation team augmented by data management, application development, and enduser organizations.

\section{Discussion}

This milestone is critical to the success of the warehouse. Its main thrust is to identify the five or six key information drivers required by management for the effective, safe, and cost-efficient management of the Savannah River Site. These requirements become the source for selecting the data that will populate the global and local warehouses. Once identified, these requirements must be bounded (for example, by priority, feasibility, or other metrics established by the IW implementation team ) to ensure a smooth and successful implementation. This may be an iterative process with successive levels of requirements. After the initial warehouse is established within the culture, the warehouse can be extended to include additional requirements.

The WSRC Strategic Plan for Business Computing identified four areas for IW support: personnel, financial, asset management, and purchasing. These are well established mainframe or core legacy applications with high-volume end-user access requirements. The financial and purchasing applications were identified in the business plan as candidates for replacement by commercial software.

\section{Action Items}

1) Identify top business informational requirements needs of SRS.

2) Identify existing data sources/usage satisfying these requirements.

3) Prioritize requirements.

4) Categorize requirements into global and/or department requirements.

\section{Dependencies}

IW.A.1

Establish IW Implementation Team 


\section{Benefits}

Establishing a warehouse oriented towards the key information requirements of the site and populating it with well defined and accurate data easily accessible by the end-user community will establish the credibility of the technology and gain user confidence. 


\section{IW.P.2 Define the IW Model}

\section{Responsible Organization}

IRM

Recommend Initiation Date

$2 \mathrm{Q} / 94$

\section{Anticipated Duration}

Nine months

\section{Resource Considerations}

The creation of the IW model involves several IRM specialties (for example, data and database management, application development, communications, and operations).

\section{Discussion}

The business requirements of IW.P.1 are the basis for creating the logical model of the warehouse. The logical model defines the schema for the global warehouse and preliminary schema for local warehouses. The purpose of the model is to establish the structure of the IW (for example, subject or functional areas), identify capacity requirements, and provide the basis for selecting the operating platform. The process of creating the model will identify a variety of issues. For example, data sources, data redundancies, and questions of ownership, sharability, and timing. All issues and questions should be resolved before moving to the next milestone, IW.P.3, Implementing the Global Warehouse. The selection of the platform is a critical issue for the warehouse. Capacity (storage, available MIPS), ease of access, and interoperability are important factors in selecting the platform. Levels of granularity are another (for example, high granularity, low granularity, zero granularity). The logical model should help resolve these questions.

\section{Action Items}

1) Create logical model of global IW.

2) Identify capacity requirements for global warehouse environment.

3) Select global IW platform.

4) Define structure or architecture for local warehouses.

\section{Dependencies}

IW.P.1

Identify and Prioritize Business Requirements for Global and Local Warehouses

\section{Benefits}

Creating the IW logical model is the lynchpin milestone of the IW roadmap. Several of the milestones and implementation/design decisions are based on the model. For example, selection of the platform, DBMS engine, tools, storage requirements, and access method are dependent on the logical model. 


\section{IW.P.3 Implement the Global Warehouse}

Responsible Organization

IRM

Recommend Initiation Date

$3 Q / 94$

\section{Anticipated Duration}

24 months

\section{Resource Considerations}

Implementing the global warehouse involves the services of all IRM organizations:

- application development groups to develop or modify existing extract code

- data management to define data standards and resolve issues of data ownership, data sources, and data definitions

network management and platform support

\section{Discussion}

Creating the processes for populating the global warehouse is the objective of this milestone. All other processes and supporting milestones lead to or support this effort. This milestone creates the processes for data extraction, data transformation, and warehouse population. The milestone presumes that the issues identified in IW.P. 2 have been resolved, the repository has been populated with the metadata, the IW tool suite has been procured, and the polices and procedures are ready for implementation. Critical to implementing the global warehouse is milestone IW.I.4, the procurement of the hardware (for example, platform and adequate disk storage) and software (for example, repository, end-user access tools, and communications protocols).

\section{Action Items}

1) Populate IW repository with initial metadata.

2) Create extraction process.

3) Create transformation process.

4) Create load process.

5) Populate initial IW.

\section{Dependericies}

IW.A.3

IW.C.1

IW.C.2
Establish IW Policies and Procedures

Establish Training for Application-development Community

Establish Training for End-User Community 
IW.I.1

Establish a Communications Network Capability Supporting the Global and Local Warehouses

IW.I.2 Establish Client SQL-Network

IW.I.4 Identify and Procure IW Support Tools and Hardware

IW.P.2 Define the IW Model

\section{Benefits}

Success of the warehouse concept is less dependent on the mechanics of populating the warehouse than on end-user ability to easily access and report on the information critical to their operations. The warehouse must be central to their reporting strategy. Having gained their confidence and the credentials of the warehouse having been established, only then will the benefits of the information warehouse become real and measurable. 


\section{IW.P.4 Expand Global Warehouse}

Responsible Organization

IRM

Recommend Initiation Date

$2 \mathrm{Q} / 96$

\section{Anticipated Duration}

24 months

\section{Resource Considerations}

The resources requirements for fully populating the global warehouse as modeled in IW.P.2 have several alternatives. First alternative is to continue the process via the original IW implementation team. Second alternative is to disband the team and transfer the responsibility to the IW support organization. A third alternative is to transfer the responsibility to the IRM application development groups with oversight by the IW support organization. Ultimately, the IRM organizations would assume responsibility for the global warehouse and underlying support processes.

\section{Discussion}

This milestone expands the global warehouse of IW.P.3 by fully populating the warehouse with the remaining business requirements identified in IW.P.1 and modeled in IW.P.2. However, the expansion should be incrementally phased to protect the warehouse and minimize end-user disruptions.

\section{Action Items}

1) Populate repository with meta-information for each iteration of data.

2) Expand processes created in IW.P.3.

3) Retune warehouse for end-user query.

4) Repeat items 1-3 as necessary to fully populate the warehouse with additional data.

\section{Dependencies}

IW.P.2

IW.P.3

Define the IW Model

Implement the Global Warehouse

\section{Benefits}

User satisfaction, faster and more accurate reporting, and reduction in maintenance backlog are some of the benefits that can be realized by a fully populated warehouse supported by a robust repository, data subscription service, and suite of data access and reporting tools. 


\section{IW.P.5 Establish Local or Remote IWs}

Responsible Organization

IRM

Recommend Initiation Date

$1 \mathrm{Q} / 95$

\section{Sponsoring Organization}

IRM

Status

Not started

\section{Anticipated Duration}

18 months

\section{Resource Considerations}

Network services, data management and application development resources.

\section{Discussion}

Where the global warehouse services the information requirements of a majority of the SRS business organizations, organizations often have a requirement for subsets of the global data. Local warehouses support these requirements, which are often geared towards specific what-if analysis. Local warehouses contain a variety of data types (for example, summaries of global data, detailed subsets of specific data, or derived data where existing data is combined to form new data). A robust computing infrastructure and communications network is a must for local warehouses. Likewise, a well constructed data distribution architecture is required. Implementing local warehouses requires data distribution tools and software.

\section{Action Items}

1) Determine specific business requirements for local warehouses.

2) Establish server requirements (for example, frequency, location, and hardware).

3) Procure hardware and software.

3) Establish data distribution techniques (for example, subscription service, downloads).

4) Establish policies and procedures for data usage.

5) Populate initial summary/department warehouse.

6) Review results of initial warehouse and modify processes as necessary.

\section{Dependencies}

IW.I.1 . Establish a Communications Network Capability Supporting the Global and Local Warehouses

IW.P.1 Identify and Prioritize Business Requirements for Global and Local Warehouses

IW.P.2

Define the IW Model

IW.P.3 Implement the Global Warehouse 


\section{Benefits}

Local warehouses often support the specific information or analytical needs of individual business organizations or users. They provide the basis for analysis and what-if modeling, while reducing the adhoc demands on the global warehouse. 


\section{IW.P.6 Establish Archival IW}

Responsible Organization

IRM

Recommend Initiation Date

$3 Q / 95$

\section{Sponsoring Organization}

IRM

Status

Not started

\section{Anticipated Duration}

12 months

\section{Resource Considerations}

Implementing an archival warehouse involves the resources and skills of several IRM organizations: operations for network support, data transfers, storage and archival, and software installation; IRM's application development staff for requirement identification, and the IW support organization for oversight and implementation.

\section{Discussion}

The archival or offline storage of warehouse data is necessary to the efficient management of the global warehouse. However, this facet of information warehousing is the least understood or supported by the current software vendors. The actual transfer of aged data is purely a mechanical function. However, the interface with the IW repository, repopulation to the active warehouse based on user demand, and yet undefined requirements will require research by the IW team or support organization.

\section{Action ltems}

1) Determine business requirements for archival warehouse.

2) Establish archival platform requirements (for example, frequency, location, and hardware).

3) Establish population/repopulation process.

4) Establish policies and procedures for data usage.

5) Populate initial archival warehouse.

6) Review results of initial warehouse and modify processes as necessary.

\section{Dependencies}

IW.P.1

Identify and Prioritize Business Requirements for Global and Local Warehouses

IW.P.2 - Define the IW Model

IW.I.5 Identify and Procure Archival Management Tools and Hardware 


\section{Benefits}

Archival warehouses usually contain data infrequently accessed or not required to support current business activities which cannot (for example, for legal reasons) be deleted. The archival warehouse often utilizes alternative forms of data storage such as tape or silos. 


\section{Client/Server Roadmap}

\section{Introduction and Destination}

Client/server ( $\mathrm{C} / \mathrm{S})$ is a term that defines the relationship of one computer to another based on their cooperation to execute a software application. There are many different configurations that are defined as client/server by the industry today, as shown in Figure 4 below, but we will use a more restrictive definition. An application is said to be a $C / S$ application when one machine, usually on a desktop, performs some of the functions of an application and requests services of other machines to provide the remainder of the application function. In the typical example of a simple C/S application, the application runs entirely on the desktopPC with SQL calls to a relational database management system running on another machine somewhere on the network. In a more complex example, some of the business logic functions of the application are performed on the desktop or client, and some are performed on a server. In this instance, the communication between the two pieces would be at a lower level than SQL and would typically be done with remote procedure calls (RPCs).

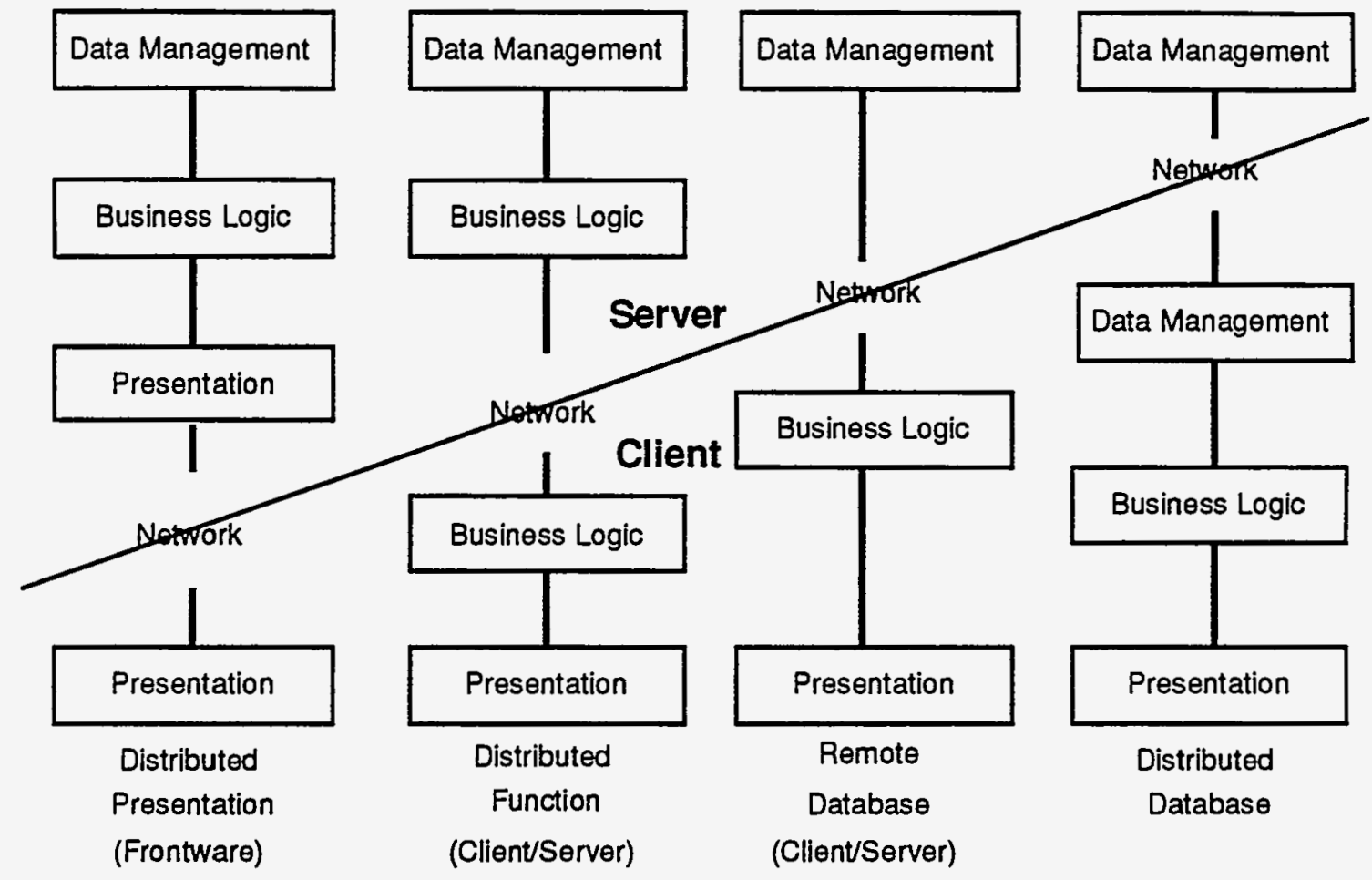

Figure 4: Sample client'server configurations.

With the reduction in price of computer hardware and the advancement of application development tools and methodologies, it has become more cost-effective for business to develop applications which utilize the increasing computing power on the desktop to provide easy-touse graphical applications. These graphical applications, with their radio button choices, pushbutton command interfaces, and their graphical presentation of information, allow end users to see clearly presented information derived from data instead of the raw data itself. A graphical application user can comprehend the application and the information being presented so that 
informed decisions can be made with less application experience and in less time. Client/server applications provide the ability to validate and reorganize the data retrieved from a remote database without impacting the availability of the same data to other users at the same time and with a reduced impact on the network. The users are able to save various views of data directly to spreausheets, word processors, and other office applications for the easy creation of graphs, charts, and reports which would be impossible to reproduce at the same cost and in the same amount of time from an old terminal-based system.

Ideally, the vast majority of applications will be developed or purchased with a client/server architecture. There would be only the occasional terminal or host-based application being constructed or maintained, but significant justification would need to be provided to management before the deviation from the norm would be allowed. Even these occasional exceptions would store their data in the site standard database so that distributed query and reporting are possible. These applications would be built by small teams made up of people with various backgrounds from both the information systems (IS) and the user/customer organizations.

The end result would be a business environment which provides full and open access to all the site data that any knowledge worker might need to perform his or her job duties. This access would be provided via a suite of application components which execute on the local workstation. This suite of components would be easily connected through drag and drop capabilities voice input, or other object-oriented technology to provide new sets of data as required by the knowledge worker. 


\section{Starting Point}

The Savannah River Site has very little experience developing client/server applications. All of the core business systems currently being maintained by IRM's application development organizations are host-based systems that run mostly on IBM or DEC VAX mainframe computers. Although Information Resource Management has made some progress in laying the groundwork for developing client/server applications and has developed production client/server systems for use inside IRM, many issues must be resolved before IRM can begin providing core business systems in this type of environment.

For the past 10 years, ADABAS, a non-relational, inverted-list database management system, has been used as the primary tool for IRM business systems. In the last three years, several systems, including the core financial systems, have been developed and implemented using DB2, IBM's relational database. No production middleware products, either from Software AG, the ADABAS vendor, or from third-party vendors currently exist to provide remote data access to ADABAS tables. This practically eliminates the possibility of using ADABAS as a server in client/server development. Middleware does exist for accessing DB2 remotely, including connectivity products such as $\mathrm{SQL}^{*}$ Connect from Oracle and database gateways from vendors such as MicroDecisionWare. DB2 also uses SQL, a standard query language supported by most relational DBMS products.

IRM has also built several Oracle applications, using both the Oracle database and Oracle tools in a mainframe terminal environment. The Oracle database has many middleware products, both from Oracle and third-parties, that allow remote data access. The Oracle database is also supported by all of the major vendors in the middleware and client/server development tool markets.

IRM has focused client/server development on the Oracle 7 database environment. A fourth generation language client/server, object-oriented, Windows-based development tool from was chosen by the IRM C/S team as the tool of choice for this type of development. A class library of reusable objects was developed by the team and used to develop the Request Management System (RMS). RMS is used internally in IRM to track and manage customer work requests and maintain information about systems currently in production or under development.

IRM has also evaluated several other client/server development environments. Recently, IRM performed an extensive evaluation of Forms 4.0, Oracle's entry into the graphical user interface, client/server environment. The evaluation team was unable to recommend Forms 4.0 as a strategic direction for department-wide client/server development due to user-interface problems, as well as the stability of the tool itself. IRM continues to pursue Forms 4.0 as an upgrade path for existing Oracle applications, and future releases of the software may resolve many of the interface and stability problems.

Today, there are many obstructions to the development of client/server applications. A vast collection of legacy applications are host-based. These applications consume a majority of application development resources for maintenance and enhancement functions. A large percentage of site data is stored in a mainframe database that is not relational and lacks remote connectivity, therefore limiting the flexibility and ease of access for reporting, query, and database expansion. Also, the current computer and network infrastructure is not sufficient to support a robust client/server operating and management environment. The WSRC Strategic Plan for Business Computing also outlines a strategy for the reduction of new system development in IRM in favor of an emphasis on purchased packages. Although the Strategic Plan calls for adherence to the computing architecture defined in the Savannah River Site Computing Architecture, which directs the move towards toward client/server applications and distributed database development, how purchased software will fit into this architecture is unclear. With a decrease in emphasis on new application development by IRM and the perceived need to 
develop custom software in the same environment as purchased applications, the future of client/server application development in IRM faces many obstacles.

While a few obstacles to client/server application development fall into the category of technical deficiencies, especially the sitewide implementation of an enterprise Novell network, the majority are either administrative, cultural, or infrastructure related. Thus, IRM is justifiably concerned with making an important tactical decision regarding a client/server database and tool. At this point, client/server application development for IRM customers is suspended while new directions and the impact of the Strategic Plan are evaluated. 


\section{Client/Server Infrastructure Milestones}

The following computing infrastructure milestones (networks, standardization, desktops. etc.) must be resolved to adopt the technology.

C/S.I.1

Inistall Robust Development Workstations

C/S.I.2

Implement Locally Distributed, Centrally Managed RDBMS Servers

C/S.I.3

Implement Single Network Transport Protocol for Client/Server

Communication 


\section{C/S.I.1 Install Robust Development Workstations}

\section{Responsible Organization}

IRM

Recommended Initiation Date

$1 \mathrm{Q} / 94$

\section{Sponsoring Organization}

IRM

Status

Started

\section{Anticipated Duration}

12 months

\section{Resource Considerations}

This milestone will require the procurement and configuration of fast CPU workstations in the Pentium class or higher with $17^{\prime \prime}$ or 21 " monitors driven by fast, high-resolution graphics cards and $16 \mathrm{MB}$ or more of RAM for each application developer and designer.

\section{Discussion}

Client/Server application developers need high-powered workstations with large, highresolution monitors and fast CPUs. The extra power is needed because of the multiple sessions running simultaneously under Windows. This part of their daily job function requires more processor speed, memory, video space, and hard disk space.

\section{Action ltems}

1) Establish functional specifications for development workstations.

2) Determine number of application developers to be equipped.

3) Prepare acquisition plan for graphical developer's workstations.

4) Competitively procure workstations/place blanket order for workstations.

\section{Dependencies}

None

\section{Benefits}

By providing the developer with these high-end workstations, IRM can shorten the amount of time required to build and test application components. The developers will experience lower overall frustration levels because they don't need to wait for the computer to finish the next job, gain increased communication levels among team members, and are allowed greater freedom to explore more complicated application models. 


\section{C/S.I.2 I Implement Locally Distributed, Centrally Managed RDBMS Servers}

Responsible Organization

IRM

Recommended Initiation Date

$1 \mathrm{Q} / 94$

\section{Anticipated Duration}

24 months

\section{Resource Considerations}

This milestone will require the purchase and installation of a number of database server hardware and software platforms to be distributed to the various geographic locations onsite. Determining the number and size of the servers is a task included in the scope of this milestone and is part of IRM's implementation of the WSRC Strategic Plan for Business Computing.

\section{Discussion}

The local database servers are controlled by a single organization. The controlling organization has satellite groups dispersed geographically to provide timely support to each of the workgroup areas. Data that is utilized heavily by one specific organization in one location is stored on a local distributed database server. In addition to the location-specific data, all commonly accessed sitewide databases are replicated for reading only on the local database server for improved performance and reduced network traffic.

\section{Action ltems}

1) Establish functionals pecifications for RDBMS servers.

2) Determine number of RDBMS servers to be purchased.

3) Prepare acquisition plan for RDBMS servers.

4) Competitively procure RDBMS servers/place blanket order for servers.

5) Create RDBMS management plan, including server system management.

6) Install RDBMS servers.

\section{Dependencies}

Networks.I.5 Install Ethernet/FDDI Network Trunks

\section{Benefits}

Provides for local workgroup data storage, centrally managed and backed up data, higher online availability of data, faster response time to data queries, lower mainframe overhead, robust site standard RDBMS for all important site data, and sitewide access to workgroup data. 


\title{
C/S.I.3 Implement Single Network Transport Protocol for Client/Server Communication
}

Responsible Organization

IRM

Recommended Initiation Date

$3 Q / 94$
Sponsoring Organization

IRM

\section{Anticipated Duration}

Three months

\section{Resource Considerations}

This milestone will require only a small manpower commitment. There are no other resources required.

\section{Discussion}

Currently a number of protocols are used for various platforms. Many software packages require some communications protocol that is not universally supported nor strategic for SRS. A single site standard protocol (for example, TCP/IP) will be used for transport of all communications between client and server.

Action ltems

1) Select protocol standard.

2) Establish procedure to enforce standard.

\section{Dependencies}

\author{
Netsys.I.8 \\ Manage IP Addresses
}

\section{Benefits}

A single network protocol would allow more efficient management of network traffic at the client workstation. Additionally, this single standard helps to establish a single connection method to numerous site data repositories, servers, and host systems, thus reducing the system management load on all of these computers. 


\section{Client/Server Administrative Milestones}

The following administrative milestones must be resolved to adopt the technology.

C/S.A.1

C/S.A.2

C/S.A.3

C/S.A.4

C/S.A.5
Develop Graphical User Interface Guidelines

Develop Naming Conventions

Enable Sitewide Data Access

Encourage Move Toward Client/Server Development

Migrate Selected Host-Based Legacy Systems to Client/Server 


\section{C/S.A.1 Develop Graphical User Interface Guidelines}

Responsible Organization

IRM

Recommended Initiation Date

$2 \mathrm{Q} / 94$

\section{Sponsoring Organization}

IRM

Status

Started

\section{Anticipated Duration}

Six months

\section{Resource Considerations}

This milestone will require the time commitment of qualified individuals to study existing interface standards and choose or derive the best set of guidelines for the site.

\section{Discussion}

All graphical applications developed in-house will have the same general look and feel. Behavior will be consistent for graphical objects from one application to another. This cannot be accomplished without eliminating character-based application user interfaces, which can't be done until character-based terminals and under powered workstations have been replaced.

\section{Action ltems}

1) Form team to establish guidelines.

2) Establish guidelines and publish team recommendations.

3) Implement policy of adherence to guidelines.

\section{Dependencies}

None

\section{Benefits}

End users will not be required to learn each application in depth and forget all former experience before they can be productive with a new application. Consistency reduces the required training time and expense significantly. The graphical nature of user interfaces increases the accuracy of work for transaction-processing applications. 


\section{C/S.A.2 Develop Naming Conventions}

\section{Responsible Organization}

IRM

Recommended Initiation Date

$2 Q / 94$

\section{Sponsoring Organization}

IRM

Status

Not started

\section{Anticipated Duration}

Six months

\section{Resource Considerations}

This milestone will require a part-time commitment of a number of individuals to study each language, database, and GUI environment to establish a naming convention compatible with other established conventions that is easily understood and adhered to.

\section{Discussion}

Naming conventions are established for the DBMS, the application development environment, and all other $\mathrm{C} / \mathrm{S}$ application components. These conventions are formalized and available to developers and users alike.

\section{Action ltems}

1) Form naming standards team from development organizations.

2) Define and publish standards.

3) Implement naming standards.

\section{Dependencies}

None

\section{Benefits}

Naming standards provide consistency among development groups so that developers can be easily reassigned from project to project with little lost productivity. 


\section{C/S.A.3 Enable Sitewide Data Access}

Responsible Organization

IRM

Recommended Initiation Date

$4 Q / 94$
Sponsoring Organization

IRM

Status

Not started

\section{Anticipated Duration}

12 months

\section{Resource Considerations}

This milestone cannot be accomplished without the commitment of a team of individuals trained in network security and authentication, middleware, and site data requirements.

\section{Discussion}

The major emphasis on this milestone is providing access to all site data repositories to which a user requires access with the single point of authentication to the network. This requires a major shift in the way application, system, and data security is handled. In order to keep the manpower required to control the access for each user at a manageable level, directory services and network authentication must be in place. This will allow a user to log in to the network once and have all systems, data, and applications to which he or she has access available without further logins or authentications.

\section{Action ltems}

1) Establish a sitewide team to develop plan.

2) Identify existing methods of access control/authentication.

3) Establish policy and procedures for sharing of site data.

4) Produce and publish recommendations.

5) Establish procedure for sitewide data access from single login.

\section{Dependencies}

Networks.I.5

Install Ethernet/FDDI Network Trunks

Netsys.P.1

Implement Single User Login to Network

C/S.I.2

Implement Locally Distributed, Centrally Managed RDBMS Servers

\section{Benefits}

This simplifies the user interface to the $C / S$ applications developed. Users will no longer have to remember multiple passwords and IDs to access their data. This milestone will simplify the management of accounts behind the scenes, making it much easier to create, update, and maintain user account information. 


\section{C/S.A.4 Encourage Move Toward Client/Server Development}

Responsible Organization

IRM

Recommended Initiation Date

$1 \mathrm{Q} / 94$

\section{Anticipated Duration}

24 months

\section{Resource Considerations}

Other than the initial time commitment of the policy team, the resources required for this milestone are negligible.

\section{Discussion}

In addition to the built-in benefits of working with the latest tools, techniques, and hardware, client/server application developers will be assigned to work on new development of highimpact systems. Those who do not make the shift to the client/server paradigm will be assigned to perform maintenance on the steadily decreasing collection of legacy code.

\section{Action Items}

1) Form team to recommend policy changes.

2) Produce list of recommended policy changes.

3) Obtain management approval of policy recommendations.

4) Establish client/server training program.

5) Establish procedure incorporating policy recommendations.

\section{Dependencies}

\section{C/S.A.3 \\ Enable Sitewide Data Access}

\section{Benefits}

With incentives provided to move toward client/server, and negative or neutral feedback for those who are not ready to change, IRM will be able to develop a highly skilled and motivated workforce. A skilled and motivated workforce will go a long way to make WSRC and the SRS a strong competitor for DOE business in the years ahead. 


\section{C/S.A.5 . Migrate Selected Host-Based Legacy Systems to Client/Server}

Responsible Organization

IRM

Recommended Initiation Date

$2 \mathrm{Q} / 94$

\section{Anticipated Duration}

48 months

\section{Resource Considerations}

This milestone will require a significant manpower commitment throughout its duration. Reducing the manpower commitment will greatly extend the elapsed time of these tasks, and may make attainment of this milestone impossible.

\section{Discussion}

All host-based systems must be analyzed to determine which can be re-engineered and moved to the $\mathrm{C} / \mathrm{S}$ platform. Once this list is determined, then sunset dates need to be established each the host application. At that time, the host-based system will be taken offline. Until this is done, it will be difficult to find the time for analysts to be retrained in the new technology. The assumption is that once a sunset date is established on a host based system, then no more new development will be done on that system. This should provide enough free time to allow some of the analysts previously working on those systems to start their training process. Either applications will be rebuilt using client/server technology, or client/server applications will be purchased.

\section{Action ltems}

1) Determine migration selection criteria.

2) Evaluate all existing systems against criteria.

3) Establish migration schedule.

4) For each application to be migrated, determine migration method.

5) For each application, create migration team and migrate application.

6) Re-evaluate selection criteria as needed.

\section{Dependencies}
C/S.A.1
C/S.A.3
Develop Graphical User Interface Guidelines
Enable Sitewide Data Access
C/S.P.1 


\section{Roadmap to the SRS Computing Architecture}

\section{Benefits}

As each application is migrated from the existing environment, the application's support personnel can be re-allocated to supporting and developing client/server applications. The enhanced ease of use of C/S applications will require less user support and training. Many of the mainframe-based systems can be eliminated, thereby freeing up CPU and DASD resources to be used by those remaining applications for which client/server migration is not a viable option. 


\section{Client/Server Culture Milestones}

The following cultural milestones must be resolved to adopt the technology. Each milestone is discussed in more detail in the following sections.

C/S.C.1

Establish Small Development Team Methodology

C/S.C.2

Establish Policies Favoring Iterative Software Development 


\section{C/S.C.1 Establish Small Development Team Methodology}

\section{Responsible Organization}

IRM

Recommended Initiation Date

$1 Q / 94$

\section{Sponsoring Organization}

IRM

\section{Anticipated Duration}

24 months

\section{Resource Considerations}

This milestone requires no additional resources over the current method of doing business. It does, however, have the potential for increasing the amount of space allocated to an individual or team to a more reasonable and workable size.

\section{Discussion}

The arrangement of office space and meeting areas is designed around the small development team. The workspace consists of a common work space / meeting area with attached individual cubes or offices. This will increase team performance and productivity. Large projects will be broken down into several small sub-projects with communication interfaces established for integration of work and reduced duplication of effort.

\section{Action Items}

1) Pilot the small team concept to develop a medium-sized $C / S$ application.

2) Publish recommendations from pilot.

3) Enlarge scale of pilot to encompass entire workgroup(s).

4) Arrange office space (cubicles) for optimizing small team projects.

5) Establish site guidelines for application developers' workspace.

\section{Dependencies}

None

\section{Benefits}

Small development teams are able to accomplish much higher quality work in a shorter period of time than large teams. Every effort made to reduce the complexity of communications between the group members will increase the performance of the group. 


\section{C/S.C.2 Establish Policies Favoring Iterative Software Development}

Responsible Organization

IRM

Recommended Initiation Date

$2 Q / 94$

\section{Anticipated Duration}

24 months

\section{Resource Considerations}

This milestone will require no additional resources over our current levels.

\section{Discussion}

Since graphical client/server development is an iterative process, the process of building an application consists of repeated prototype refinements. The measurement of progress is based on the level of user satisfaction with the current iteration of the prototype. Object-oriented methodologies and programming techniques will allow increased scalability of an application project so that the first production release will be small, but each iteration will build on the core to expand to a large group of related components that are easily maintainable.

\section{Action Items}

1) Identify milestones in the iterative lifecycle.

2) Restructure project management and scheduling for iterative milestones.

3) Remove remnants of waterfall methodology thinking.

\section{Dependencies}

None

\section{Benefits}

Strict schedules and budgets will prevent long development cycles. Cost saving applications will be available earlier than was possible using the old methodology. Systems will more closely match the needs of end-user organizations. 


\section{Client/Server Process Milestones}

The following work flow milestones must be resolved to adopt the technology.

C/S.P.1

Implement Standard Development Methodology and Tools Across Site

C/S.P.2 Integrate Software Tools, Development Methodology, Project, and Configuration Management Products 


\section{C/S.P.1 Implement Standard Development Methodology and Tools Across Site}

Responsible Organization

IRM

Recommended Initiation Date

$1 Q / 94$

\author{
Sponsoring Organization
}

IRM

Status

Started

\section{Anticipated Duration}

12 months

\section{Resource Considerations}

The software tools used in designing and constructing client/server applications are extremely powerful. To run this software, developers will need very fast workstations (Pentium or PowerPC class or faster) with at least 32 MB of RAM. CASE tool users will need at least 21" monitors and graphics cards capable of displaying very complex diagrams at resolutions of 1600 $\times 1200$ or greater at high scan rates. These workstations will also require large local hard drives to act as temporary storage of the models to minimize network traffic as much as possible or high speed (FDDI or ATM) network access to high performance file servers. Developers who do not use CASE tools will require only the standard high-end developers workstation.

\section{Discussion}

With the establishment of a single set of tools and a development methodology, developers and integrators would be able to move from project to project with minimal to no additional training. There will also be no time required to adjust to a new way of working. The result will be a more flexible and efficient workforce.

\section{Action Items}

1) Identify organizations affected by standards to form standards team.

2) Identify methodology(ies) suitable for client/server development.

3). Identify tools suitable for automating methodology(ies).

4) Identify development language, libraries, etc.

5) Publish list of standard tools.

6) Establish procedures requiring adherence to the standards.

\section{Dependencies}

C/S.I.1 Install Robust Development Workstations

\section{Benefits}

Developers will not need to adapt to different methods and learn new tools when they are moved from project to project. Significant time and training money can be saved, and increased flexibility of the IRM organization can be realized. 


\section{C/S.P.2 Integrate Software Tools, Development Methodology, Project, and Configuration Management Products}

Responsible Organization

IRM

Recommended Initiation Date

$1 Q / 94$

\section{Anticipated Duration}

18 months

\author{
Sponsoring Organization
}

IRM

Status

Started

\section{Resource Considerations}

This milestone will require the purchase of software tools such as repository tools, CASE tools, and configuration management tools.

\section{Discussion}

The system development methodology that will be utilized by software developers is a commercially available package. It will have built in links to configuration management, object management, project management, and software development tools that ensure compliance. The SDM will be available online with built-in help facilities. The SDM will be flexible to allow for various categories of applications, so that different rules are applied to different levels of development. Low impact maintenance of objects will be very loosely controlled, while development of mission-critical applications will demand more rigorous requirements.

The level of automation of the software specification and development cycle will be extremely high. This will reduce the number of places where errors can be introduced early in the cycle and greatly increase software quality. Integrated into this same package suite will be some project management tools to assist the project leader in tracking project progress. The number of products composing the developers tool suite will be limited so that the developer will be able to achieve a higher degree of skill in each tool instead of a limited knowledge of many tools.

\section{Action ltems}

1) Form a team to develop specifications for all parts of the environment.

2) Develop acquisition plan for the software to set up environment.

3) Implement software to establish environment.

4) Establish procedure for usage of environment.

\section{Dependencies}

C/S.P.1 - Implement Standard Development Methodology and Tools Across Site

\section{Benefits}

This will allow small development teams to work efficiently yet still provide solid version control and project management to meet all DOE order requirements. This environment will 
minimize the retraining required as a developer moves from one group to another group because of an online, automated developers' environment. 


\section{Client/Server Technology Milestones}

The following technology evaluation and pilot project milestones must be resolved to adopt the technology.

C/S.T.1 Implement Standard RDBMS Accessibility

C/S.T.2 Implement Graphical Report Generators and Query Tools

C/S.T.3

C/S.T.4

Implement Nonrelational Database Access Through SQL Gateway

Implement Declarative Referential Integrity, Triggers, and Stored

Procedures Supported by RDBMS

C/S.T.5 Implement Monitoring Tools

C/S.T.6 Implement Cross-platform Two-phase Commit Regardless of Vendor 


\section{C/S.T.1 Implement Standard RDBMS Accessibility}

Responsible Organization

IRM

Recommended Initiation Date

$2 \mathrm{Q} / 94$
Sponsoring Organization

IRM

Status

In progress

\section{Anticipated Duration}

18 months

\section{Resource Considerations}

Manpower required to perform the conversion of data currently residing in ADABAS and other various flat file formats, and to replace or modify applications that work with the data in its non-relational format.

\section{Discussion}

All applications can be assured access to any required data through standard sitewide links between the standard database or gateways. No effort is expended writing extract routines to gather data from incompatible databases once the data is migrated to the standard RDBMS. Only extracts for the historical need of the site data warehouses will be done.

\section{Action Items}

1) Establish standard RDBMS connection standard specifications.

2) Compete standard for each platform tier.

3) Implement standard software on all platforms.

\section{Dependencies}

C/S.I.2 Implement Locally Distributed, Centrally Managed RDBMS Servers

Netsys.P.1 Implement Single User Login to Network

\section{Benefits}

When all site data is stored in a standard RDBMS, the cost of obtaining data as required for new applications will be greatly reduced. Most cost reductions will result from eliminating the need to write routines to extract data and convert it into a format that is acceptable to the particular application environment. 


\section{C/S.T.2 Implement Graphical Report Generators and Query Tools}

Responsible Organization

IRM

Recommended Initiation Date

$3 \mathrm{Q} / 94$

\author{
Sponsoring Organization
}

IRM

\section{Status}

Not started

\section{Anticipated Duration}

Six months

\section{Resource Considerations}

Tools must allow developers and users to create shared reports, graphs, and queries. Tools must allow users to create their own ad hoc queries, reports, graphs, etc.

\section{Discussion}

Since user requirements are always changing to reflect changing business requirements, the set of reports, graphs, data extracts, etc. available to the application user must be infinite. Graphical and easy-to-use report, extract, view, and query tools must be provided to users so they can get the information they need to make business decisions on their own or use the growing set of user-defined queries in the common repository.

\section{Action Items}

1) Evaluate graphical reporting/query/extract tools, and write bid specifications.

2) Bid and purchase tools.

3) Pilot integration of tools in application.

4) Implement standard end-user tool set.

\section{Dependencies}

Netsys.I.1

Install Robust Client Workstations

C/S.A.1

Develop Graphical User Interface Guidelines

\section{Benefits}

The time that would normally be used to build applications to produce every report that could ever be desired from the application's data can be eliminated from the project schedule. With end user reporting tools, each user can quickly and easily build any custom report he/she wishes. 


\section{C/S.T.3 I Implement Nonrelational Database Access Through SQL Gateway}

Responsible Organization

IRM

Recommended Initiation Date

2Q/94

\author{
Sponsoring Organization
}

IRM

Status

Not started

\section{Anticipated Duration}

Six months

\section{Resource Considerations}

A software product must be purchased to allow SQL access to all non-relational database systems in which site data resides. Hardware may also be required to operate this gateway software, depending on the product chosen.

\section{Discussion}

The data that remains in non-relational databases (that is, ADABAS) must be made available to clients. Access is accomplished transparently through an SQL gateway so that the non-relational nature of the database is hidden from the client.

\section{Action ltems}

1) Establish bid specifications for SQL gateway to ADABAS.

2) Put out for bid based on specifications.

3) Implement gateway software.

\section{Dependencies}

None

\section{Benefits}

Although the ultimate goal is to have all site data available in relational format, the large quantity of data that currently resides in ADABAS can be used by client/server applications before the long and costly conversion to a relational format. 


\section{C/S.T.4 Implement Declarative Referential Integrity, Triggers, and Stored Procedures Supported by RDBMS}

Responsible Organization

IRM

Recommended Initiation Date

$2 \mathrm{Q} / 94$
Sponsoring Organization

IRM

Status

Not started

\section{Anticipated Duration}

18 months

\section{Resource Considerations}

DBAs must be trained in the use of triggers and stored procedures to enforce business rules.

\section{Discussion}

It is the responsibility of the DBA to enforce the rules of the business at the database level. To accomplish this task, declarative referential integrity is available through the RDBMS. Triggers and stored procedures are available to code the more complex business rules. The developer need worry only about the manipulation of data for transaction processing, data entry, and data extraction for query and reporting.

\section{Action ltems}

1) Upgrade all site databases to support RI, triggers, and stored procedures.

2) Define policy to maintain business rules at the RDBMS.

3) Define criteria for duplication of business rules within the application.

\section{Dependencies}

None

\section{Benefits}

With the wide availability of third party tools for manipulating data on an RDBMS, it will become increasingly difficult to enforce integrity constraints outside of the RDBMS. With the server maintaining integrity, all security loopholes will be closed. Addition of this code to the client application may be done for performance and network bandwidth reasons. 


\section{C/S.T.5 Implement Monitoring Tools}

Responsible Organization

IRM

Recommended Initiation Date

2Q/94
Sponsoring Organization

IRM

Status

Not started

\section{Anticipated Duration}

18 months

\section{Resource Considerations}

Hardware and software must perform the tasks of monitoring all of the pieces that make up the client/server application environment. This includes the RDBMS, database server platform, network, and client hardware and software processes.

\section{Discussion}

With the multitude of points where an application could be adversely affected, tools must be be found to present in a graphical and intuitive fashion the current status and trends of all of the pieces of the client/server environment.

\section{Action ltems}

1) Establish criteria for monitoring tools.

2) Procure monitoring tools or suites.

2) Implement tools.

\section{Dependencies}

Networks.I.5 Install Ethernet/FDDI Network Trunks

Netsys.I.1

Install Robust Client Workstations

\section{Benefits}

Graphical network, server, and client monitoring tools will enable support personnel to quickly pinpoint problems in the complex client/server environment. Without such tools, it would be impossible to support and maintain a sitewide client/server application. 


\title{
C/S.T.6 I Implement Cross-platform Two-phase Commit
} Regardless of Vendor

Responsible Organization

IRM

Recommended Initiation Date

$4 \mathrm{Q} / 95$

Anticipated Duration

24 months

\section{Resource Considerations}

Additional network bandwidth may be required to support timely two-phase commit. No other resources will be required.

\section{Discussion}

This milestone indicates that distributed transactions will be supported between any databases in use onsite with two-phase commit. Two-phase commit will ensure data integrity even when the data to be updated resides on two different database servers in different site locations.

\section{Action Items}

1) Establish definition of two-phase commit.

2) Write bid specifications for upgrades to site standard RDBMSs to include this function.

3) Procure upgrades.

4) Implement upgrades.

\section{Dependencies}

C/S.T.1

\author{
Implement Standard RDBMS Accessibility
}

\section{Benefits}

Data integrity can be assured using two-phase commit. Decisions can be made based on data in different databases with assurance that the information is accurate. 


\section{Multimedia Roadmap}

\section{Introduction and Destination}

Multimedia is a collection of technologies that combines the formats of text, voice, video, audio, image, and animation for delivery to workstations, specialized platforms, or existing standard audiovisual equipment. While the individual functions incorporated in multimedia have been in the consumer domain for decades (television, VCR, telephone, cameras, tape recorders), their convergence at the computer desktop for interactive and simultaneous use has created the opportunity for a revolution in information access.

The major innovation in interactive multimedia will be the ability to access it in the same manner as existing data. When audio, video, image, and motion are treated as conventional data types, the access to this information can be as effortless as access to today's word processing files. Use of the workstation to effectively manage and assist users in linking the various information types on an ad hoc basis is the key to bringing multimedia into the mainstream of office and business computing.

Multimedia enables presentation and user retention of information in far greater detail and depth than through conventional means, and provides for greater flexibility. The addition of color, voice, music, and motion to information serves both to capture the attention of the recipient and to enhance the value of the information.

Multimedia's capabilities are not limited to the presentation of data but can allow user manipulation of the data, interactive multimedia. Interactive multimedia allows for nonlinear navigation through the information; that is, direct access to specific content, cross-references, and the ability to accelerate or slow down animations, zoom in on images, and combine voice annotation with otherwise static data.

Multimedia data exchange will extend beyond the sharing of applications in a desktop product environment. A navigable document format becomes the basic token or envelope for routing, presentation, markup, and archival of information. It will not necessarily transmit information in a single file but encompass a suite of data and product standards that enable seamless transfer and storage of the information in editable, searchable, and presentation formats without conversion by the user. The format will include index and search tools, as well as navigational tools such as hyperlinks and ties to the applications that created the embedded data.

The SRS multimedia workstation of 1995 will be fully digital; that is, all the audio, video, and other information will be stored digitally on local or remote disks, and will be delivered via an application to the workstation monitor and internal sound equipment. Information Resource Management (IRM) and other site organizations, particularly Training Integration and Technical Publications, are evaluating and piloting fully digital multimedia technology projects to determine multimedia's potential in the business and office environment. 


\section{Starting Point}

The majority of the technology and infrastructure items described herein did not exist two years ago, and will likely be undergo significant changes over the next five years. Most current desktop platforms at the Savannah River Site are not multimedia-capable. While Macintosh systems have native sound playback capability, most are not equipped for sound capture. Audio capability for Window's users will likely require an additional board. High-speed networking is an essential component of networked multimedia so that the data-intensive audio and color image/motion files can be centrally stored and accessed on demand. Only $40 \%$ of the site workstations today are adequately networked (Ethernet-attached at $10 \mathrm{mbit} / \mathrm{sec}$ ) for basic desktop multimedia (audio and color image). High performance multimedia (motion video to simultaneous users) will require $100 \mathrm{mbit} / \mathrm{sec}$ connectivity and backbones. Video playback software has recently become available in the marketplace for both Macintosh and Windows systems, but the ability to capture such files requires substantial upgrades to workstations.

The deployment of SRS-developed multimedia applications depends on coordinated improvements to three areas of the computing environment:

Decisions to support uniformity in software and workstations

Availability of authoring tools and multimedia capture stations (Figure 5)

Upgrades to network, desktop workstations, and support infrastructure (Figure 6)

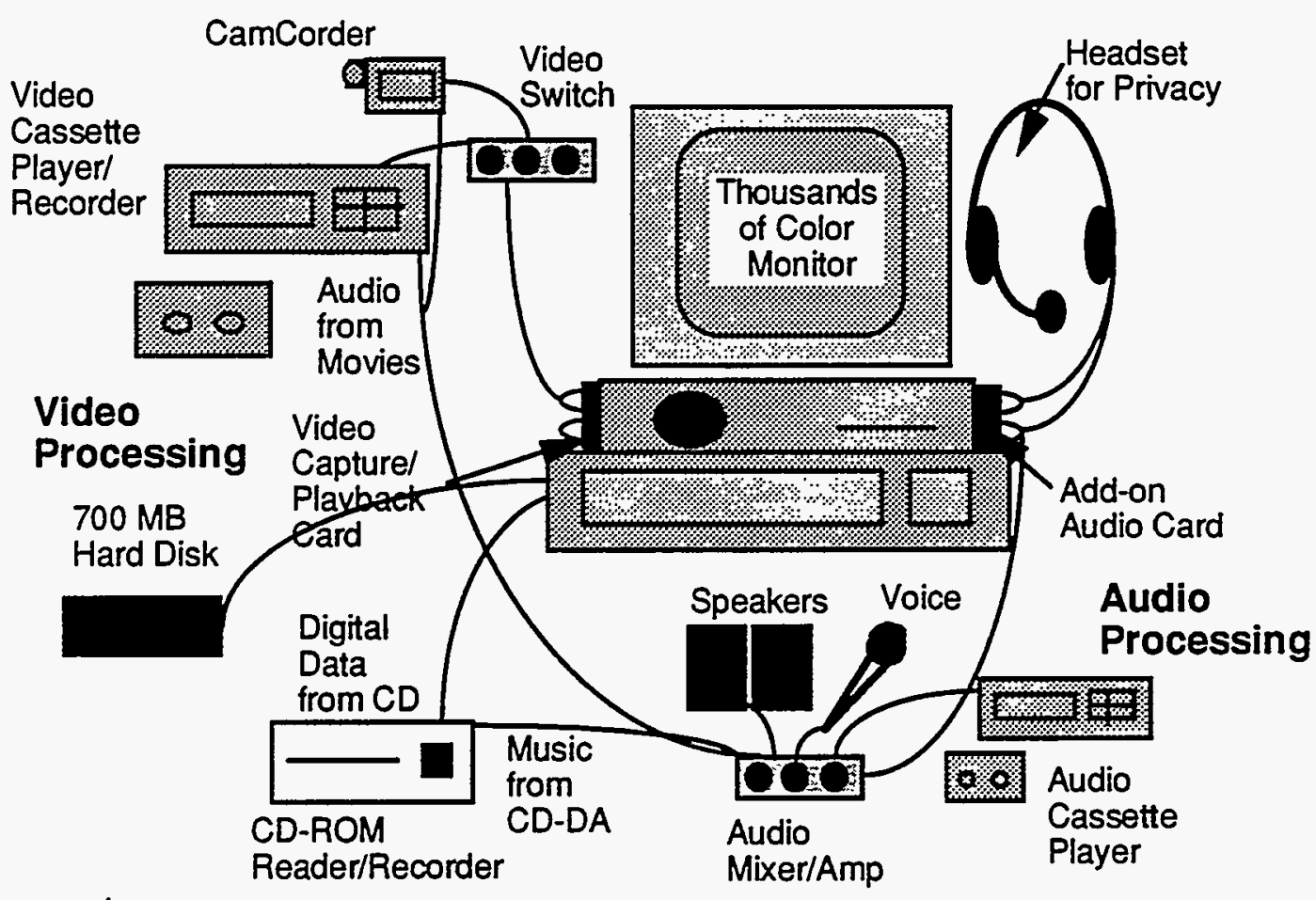

Figure 5: Multimedia capture workstation. 


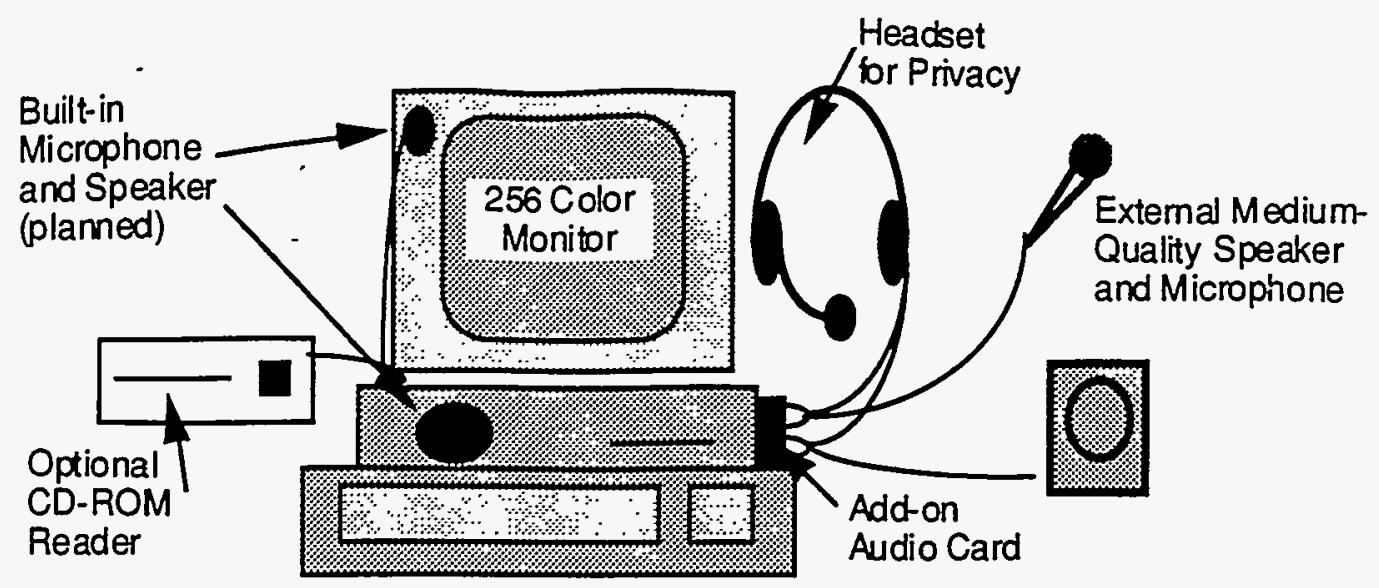

Figure 6: Multimedia playback workstation.

When multimedia applications begin to require access by a wider user base, an investment must also be made in infrastructure improvements. While nearly $40 \%$ of the site's desktop office workstations are currently networked, the original WISDOM design requirements written in 1990 (before multimedia was considered a reality) are not currently sized or configured for effective multimedia support. An immediate need will be the requirement for tens of gigabytes ( $1 \mathrm{~GB}=1,000 \mathrm{MB}$ ) of disk storage on area and workgroup servers. It is unlikely that all of this storage will be provided through magnetic, online storage. Sitewide $C D-R O M$ services and possibly rewritable optical disk will be established for access to pre-recorded titles as well as locally written applications. A determination will be made for the appropriate, use of CD-ROM versus optical disk, as well as which data types are more effective when accessed from local disk.

By late 1994, an evaluation and selection of standard data formats, desktop applications, and technologies should begin for all multimedia data types, with preliminary standards in place during 1995. Once the appropriate technology standards are identified, work will begin to identify compliant products that maximize adherence and the probability of cross-platform interoperability. 


\section{Multimedia Infrastructure Milestones}

The following milestones infrastructure must be resolved to adopt the technology in an enterprise manner.

MMedia.I.1

MMedia.I.2

MMedia.I.3

MMedia.I.4

MMedia.I.5

MMedia.I.6

MMedia.I.7

MMedia.I.8
Dẻvelop Multimedia Principles

Adopt Cross-platform Fonts and Image Formats

Establish Navigable Compound Document Format

Enable Routine Capture and Playback of Cross-platform Audio Implement Storage, Multimedia Servers, CD-ROM, and Improved Monitors

Enable Playback Motion Video at Desktop Implement Workgroup Video-capture Stations

Implement Cross-platform Authoring Tools 


\section{MMedia.I.1 Develop Multimedia Principles}

Responsible Organization

IRM, Training, MSD

Recommended Initiation Date

$1 \mathrm{Q} / 94$

\section{Anticipated Duration}

Nine months

\section{Resource Considerations}

Team effort, consensus, and review by a consultant.

\section{Discussion}

Principles are developed to lay the foundation for a unified approach to the implementation of a new architectural component. Initial bounds on the applicability and business cases for multimedia will be determined here.

\section{Action ltems}

1) Identify the participants in the principles development group.

2) Research successes in industry regarding multimedia.

3) Identify three-year objectives and scope.

4) Determine initial set of supported data types.

5) Write multimedia principles and route for review.

6) Gain endorsement of multimedia principles from stakeholders.

7) Determine impact of multimedia principles on site architecture.

\section{Dependencies}

MMedia.T.1 Pilot Multimedia Enablement of Desktop Products

\section{Benefits}

Since multimedia technology is rapidly evolving and is characterized by new products with short lives, some guidance for selection of technologies and products based on business needs is needed. The principles will limit the initial applications and product selections to those which offer significant benefit and adhere to site infrastructure guidelines. 


\section{MMedia.I.2 Adopt Cross-platform Fonts and Image Formats}

Responsible Organization

IRM, MSD

Recommended Initiation Date

$1 Q / 94$

\section{Sponsoring Organization}

IRM

\section{Status}

Started

\section{Anticipated Duration}

18 months

\section{Resource Considerations}

Team consensus, DMS support considerations. Sitewide license of desktop software $\sim \$ 50 /$ user.

\section{Discussion}

Multimedia deployment cannot proceed until the basic ability to accurately interchange textual and image information exists. Currently, the lack of standardization in fonts prevents accurate interchange of word processing files, and even fonts with the same names from different manufacturers will cause a document to repaginate and/or have different line endings when exchanged with another user. It is anticipated that the bulk of existing paper documents will be scanned into image format for retrieval at the desktop. Infrastructure support including file format and applications software must be provided. This milestone also depends on the site Document Management System (DMS) expansion project.

\section{Action Items}

1) Select Adobe ATM or Microsoft True Type technology.

2) Identify core set of a dozen site standard typefaces.

3) Determine image file format and mechanisms for cross-platform use.

4) Procure associated products.

5) Propagate products to users.

\section{Dependencies}

MMedia.I.1

Develop Multimedia Principles

Office.I.2

Install Online Scanners

Office.I.7

Implement Viewers/Translators

Netsys.P.3

Implement Software License Management

\section{Benefits .}

Text and image formats are the fundamental data types for any information exchange. The ability to exchange fully-formatted or image documents must exist as a basis for document routing, and electronic document management. While these data types are not typically considered to be multimedia, having this infrastructure in place before widespread deployment of multimedia will eliminate the need to consider these data type standards within the realm of multimedia data types, and limit multimedia integration issues to the newer data types. 


\section{MMedia.I.3 Establish Navigable Compound Document Format}

Responsible Organization

IRM, MSD

Recommended Initiation Date

$2 \mathrm{Q} / 94$

\section{Sponsoring Organization}

IRM

Status

Not started

\section{Anticipated Duration}

18 months

\section{Resource Considerations}

IRM analysis to identify key business requirements.

\section{Discussion}

A navigable document format becomes the basic token or envelope for routing, presentation, markup, and archival of information. Compound document architectures such as SGML lay the foundation for such capabilities but are not currently multimedia-capable. Hytime and HTML are emerging extensions for multimedia to the SGML standard.

\section{Action Items}

1) Begin participation on ANSI and DOE-wide steering committees.

2) Determine basic requirements.

3) Complete requirements based on pilots.

4) Procure associated products.

5) Propagate products to users.

\section{Dependencies}

MMedia.I.1

Develop Multimedia Principles

MMedia.I.2

Adopt Cross-platform Fonts and Image Formats

MMedia.T.1

Pilot Multimedia Enablement of Desktop Products

MMedia.T.2

Pilot Navigable Compound Documents

\section{Benefits}

A navigable document format becomes the basic token or envelope for routing, presentation, markup, and archival of information. It will not necessarily transmit information in a single file, but will encompass a suite of data and product standards that enable seamless transfer and storage of the information in editable, searchable, and presentation formats without conversion by the user. 


\title{
MMedia.l.4 Enable Routine Capture and Playback of Cross- platform Audio
}

Responsible Organization

\author{
Sponsoring Organization
}

IRM

IRM

Recommended Initiation Date

Status

$2 \mathrm{Q} / 94$

Not started

\section{Anticipated Duration}

12 months

\section{Resource Considerations}

$\$ 150$ per workstation for sound cards for IBM (and clone) PCs. Inclusion of audio software in WISDOM standard.

\section{Discussion}

Audio capture and playback is the first multimedia data type planned for routine use. It is widely available in consumer PCs. The next set of workstations procured for the site should be specified to include audio capture and playback hardware. The use of audio at the desktop will begin the shift from the reliance on printed information to the use of multimedia data which may have no printable counterpart. Administrative and technical issues raised by audio deployment must be resolved before further routine use of multimedia can continue.

\section{Action ltems}

1) Determine functional specifications for audio add-ons.

2) Complete competitive bid of site-standard audio add-ons.

3) Procure and install audio hardware and software.

4) Complete training for use of audio add-ons.

\section{Dependencies}

MMedia.I.5

Implement Storage, Multimedia Servers, CD-ROM, and Improved Monitors

MMedia.T.1 Pilot Multimedia Enablement of Desktop Products

Office.I.8 Integrate Voice-mail and E-mail Systems

\section{Benefits}

Audio, typically in the form of voice annotation, is the first common form of multimedia data in desktop office products. SRS should take advantage of this as the initial step into full multimedia workstation implementation. 


\section{MMedia.l.5 Implement Storage, Multimedia Servers, CD-ROM, and Improved Monitors}

Responsible Organization

IRM

Recommended Initiation Date

$2 \mathrm{Q} / 94$

\section{Sponsoring Organization}

IRM

\section{Status}

Not started

\section{Anticipated Duration}

24 months

\section{Resource Considerations}

$\$ 8$ million for server and workstation improvements. Major modifications to WISDOM scope.

\section{Discussion}

Multimedia implementation will require standardization and support of infrastructure items including fonts, document standards, sound cards, better monitors, and additional server capabilities.

\section{Action Items}

1) Provide support to procurement of identified items.

2) Establish policy on backup of multimedia data.

3) Determine applicability of dedicated multimedia servers.

4) Improve monitors to 256 or more colors.

5) Acquire additional disk space, $C D-R O M$, and/or rewritable optical storage.

\section{Dependencies}

MMedia.I.1

Netsys.I.1

Netsys.I.9

Netsys.C.3

\section{Develop Multimedia Principles}

Implement Robust Client Workstations

Implement Robust File/Print/Application Services

Save Data to Network

\section{Benefits}

Having software and data available from the server (not from the workstation) will keep all workstations and applications in synchronization. Server-based capabilities must be improved to meet new workstation requirements. Monitors with better color resolution and memory will improve performance and enhance multimedia display. 


\section{MMedia.I.6 Enable Playback Motion Video at Desktop}

\section{Responsible Organization}

IRM, Video Services

Recommended Initiation Date

$1 Q / 95$

\section{Sponsoring Organization}

IRM

\section{Status}

Not started

\section{Anticipated Duration}

12 months

\section{Resource Considerations}

Adequate network bandwidth to the desktop (10 mbit/sec). Monitor upgrades to support thousands of colors ( $\$ 250$ per workstation; see Netsys Infrastructure).

\section{Discussion}

The ability to play back motion video at the desktop is the short-term goal of multimedia enablement. Video delivery will challenge both the network and the workstation capacities due to its demands for network bandwidth and computational resources in orders of magnitude beyond those required for text-based applications. Commercial databases and applications are becoming multimedia capable, and SRS should deploy this technology in selected areas such as training.

\section{Action ltems}

1) Determine cross-platform video format.

2) Determine impact on LAN bandwidth and WISDOM servers.

3) Procure and install associated software.

\section{Dependencies}

MMedia.I.2

Adopt Cross-platform Fonts and Image Formats

MMedia.I.4 Enable Routine Capture and Playback of Cross-platform Audio

MMedia.I.5 Implement Storage, Multimedia Servers, CD-ROM, and Improved Monitors

Networks.I.5 Install Ethernet/FDDI Network Trunks

Netsys.I.1 Install Robust Client Workstations

\section{Benefits}

Motion video enablement is the foundation for additional applications. Its availability can enable other applications such as training and desktop videoconferencing. 


\section{MMedia.l.7 Implement Workgroup Video-capture Stations}

Responsible Organization

IRM, Video Services

Recommended Initiation Date

$3 Q / 95$
Sponsoring Organization

IRM

\section{Status}

Not started

\section{Anticipated Duration}

Six months

\section{Resource Considerations}

$\$ 1$ million for sitewide hardware upgrades, or $\$ 15,000$ per commodity-center workstation.

\section{Discussion}

Due to the expected cost of capturing and editing video clips, it is expected that departmental stations will be provided for video capture, much the same way copiers are available to workgroups. Alternatively, video capture services could be provided through commodity centers where video professionals would help users match their needs to the appropriate technology or equipment.

\section{Action ltems}

1) Determine preferred configuration for equipment.

2) Purchase equipment via blanket orders.

3) Create IRM support teams for video services.

\section{Dependencies}

Netsys.P.2 Implement Distributed Workgroup Management

\section{Benefits}

By standardizing and consolidating video capture functions, a savings will be realized in equipment costs and support. 


\section{- MMedia.I.8 Implement Cross-platform Authoring Tools}

Responsible Organization

IRM, MSD

Recommended Initiation Date

$3 \mathrm{Q} / 95$
Sponsoring Organization

IRM

Status

Not started

\section{Anticipated Duration}

12 months

\section{Resource Considerations}

$\$ 1$ million for software licenses, $\$ 200$ per seat.

Likely to be deployed on department basis as opposed to every desktop.

\section{Discussion}

Authoring tools, which consolidate multimedia data into self-contained applications, will become easier to use and commonplace at the desktop. Inclusion of this capability as a standard is important. It does not replace the adhoc philosophy of access to multimedia on an as-needed basis by desktop users, but supplements that capability by making certain sets of multimedia data portable. The use of authoring tools provides packaging for associated files of multimedia data in applications such as training and presentations, ensuring the integrity of all the data and its links.

\section{Action ltems}

1) Establish site standard.

2) Integrate existing assets into authoring toolsets.

3) Roll-in authoring tools to WISDOM.

\section{Dependencies}

MMedia.I.5 Implement Storage, Multimedia Servers, CD-ROM, and Improved Monitors

Netsys.P.3 Implement Software License Management

\section{Benefits}

By standardizing and consolidating authoring software, a savings will be realized in license costs and support. The use of authoring tools to easily package collections of multimedia to points on- and offsite will assure that there are no omissions from the data. 


\section{Multimedia Administrative Milestones}

The following administrative milestones must be resolved to adopt the technology.

MMedia.A.1

MMedia.A.2

MMedia.A.3
Approve Use of Multimedia Data Types

Formulate Policy for Authentication of Nonprintable Data Types

Determine Business Case for Desktop Video Capture 


\section{MMedia.A.1 Approve Use of Multimedia Data Types}

Responsible Organization

Computer Security, Site Legal

Recommended Initiation Date

$2 \mathrm{Q} / 94$

\section{Sponsoring Organization}

IRM

\section{Status}

Not started

\section{Anticipated Duration}

12 months

\section{Resource Considerations}

Participation by Computer Security, Site Legal, and Management Practices, and review by independent consultants.

\section{Discussion}

Audio and video capture devices used in conjunction with multimedia development may cause concerns regarding protection of information, audit capability, labeling of information, and the connection of recording devices to computer and/or telephone equipment.

\section{Action ltems}

1) Develop management policy on multimedia data.

2) Determine new requirements for access control and physical security.

3) Determine new requirements labeling of information.

4) Identify and make changes to Computer Security Principles.

\section{Dependencies}

MMedia.I.1

Netsys.A.2
Develop Multimedia Principles

Define Security Principles

\section{Benefits}

Early identification of the impact of multimedia data and capture devices on vulnerability and protection of data will ensure that widespread deployment of multimedia will proceed with approval from all affected oversight organizations, and will be designed to maximize access to information without threatening the security. 


\section{MMedia.A.2 Formulate Policy for Authentication of Nonprintable Data Types}

Responsible Organization

MSD

Recommended Initiation Date

$3 \mathrm{Q} / 94$

\section{Anticipated Duration}

12 months

\section{Resource Considerations}

Review by independent consultants.
Sponsoring Organization

MSD

Status

Not started

\section{Discussion}

The presence of audio and video data which have no printable archival format is likely to cause concern for records management procedures. Industry and national standards are lacking in this area. Since the recognized standards for paper archival have not been updated in over a decade despite the advent of image systems, that same delay is expected for the newer data types such as audio and video. By monitoring and adhering to emerging trends in industry, the site can establish operating guidelines for internal use that can likely be grandfathered upon the establishment of national standards.

\section{Action ltems}

1) Research industry and NARA activities.

2) Draft site policy for authentication of non-printed data.

3) Gain Legal and DOE management approval of policy.

\section{Dependencies}

MMedia.1.2

MMedia.A.1

Netsys.A.2
Adopt Cross-platform Fonts and Image Formats

Approve Use of Multimedia Data Types

Define Security Principles

\section{Benefits}

Early identification of the impact of multimedia data on records management practices will ensure that widespread deployment of multimedia will proceed with approval from all affected oversight organizations. 


\section{MMedia.A.3 Determine Business Case for Desktop Video Capture}

Responsible Organization

IRM

Recommended Initiation Date

$1 \mathrm{Q} / 96$

\section{Anticipated Duration}

12 months

\section{Resource Considerations}

$\$ 4$ million (potentially).

\section{Sponsoring Organization}

IRM

Status

Not started

\section{Discussion}

A decision whether or when to encourage routine use of desktop video capture will be made. It is costly but may become integrated into new-generation PCs. Alternative uses of the site telecommunications system may provide desktop video through telephony equipment or other means. While desktop video capture cannot be justified for all users in the early stages of multimedia deployment, eventual analysis will determine whether to continue this practice on a departmental or commodity center basis, or whether the emergence of new technologies makes ubiquitous deployment cost-beneficial.

\section{Action ltems}

1) Determine compatible products and implementation costs.

2) Determine applications requiring desktop video and value.

3) Make business case for desktop video.

\section{Dependencies}

MMedia.T.5 Pilot Desktop Videoconferencing

\section{Benefits}

If desktop video can be deployed for under $\$ 250$ per workstation, and tie-in is made to commercial videoconference systems, the site can save millions in travel costs. Even for site-only videoconferencing, significant savings can be achieved in reduced travel time by employees across the site. 


\section{Multimedia Process Milestones}

The following work flow milestones must be resolved to adopt the technology.

MMedia.P.1

Manage Stored Digital Video Clips

MMedia.P.2

Implement Routine Use of Multimedia Data in Office Documents 


\section{MMedia.P.1 Manage Stored Digital Video Clips}

Responsible Organization

IRM, MSD, Video Services

Recommended Initiation Date

$3 \mathrm{Q} / 95$

\section{Sponsoring Organization}

IRM

Status

Not started

\section{Anticipated Duration}

Six months

\section{Resource Considerations}

Stakeholders from affected organizations will contribute to this process analysis.

\section{Discussion}

Policies and procedures must be established for the treatment of video data in the same manner as numerical and text information. Data ownership and distribution mechanisms must be put in place. Migration issues regarding the information warehouse will be considered, as well as the establishment of backup, access security, and data reuse guidelines.

\section{Action Items}

1) Expand WISDOM servers and/or procure video servers.

2) Create or acquire video assets.

3) Determine appropriate uses of video clips in business environment.

\section{Dependencies}

MMedia.I.5

Implement Storage, Multimedia Servers, CD-ROM, and Improved Monitors

MMedia.I.6

Enable Playback Motion Video at Desktop

MMedia.I.7

Implement Workgroup Video-capture Stations

Netsys.C.3

Save Data to Network

\section{Benefits}

Routine use of video services will reduce the dependency on meetings, presentations, and oneon-one demonstrations. Multimedia data is expensive to acquire and requires massive disk storage, but is potentially invaluable when it can be found, delivered, and reused. 


\section{MMedia.P.2 Implement Routine Use of Multimedia Data in Office Documents}

Responsible Organization

IRM, MSD

Recommended Initiation Date

$1 Q / 95$

\section{Sponsoring Organization}

IRM

Status

Not started

\section{Anticipated Duration}

24 months

\section{Resource Considerations}

Stakeholders from affected organizations will contribute to this process analysis.

\section{Discussion}

The end-user support groups and customers must use multimedia to gain its benefits. Training and operational procedures should be adapted to utilize the technology. Analysis should determine the high-payback areas for multimedia deployment and used to decide when widespread deployment of such applications is justified.

\section{Action items}

1) Re-evaluate multimedia enablement of existing applications.

2) Include multimedia capabilities in applicable procurement specifications.

\section{Dependencies}

MMedia.I.1

MMedia.I.5

Networks.A.4

\section{Develop Multimedia Principles}

Implement Storage, Multimedia Servers, CD-ROM, and Improved Monitors

Obtain Computer Security Endorsement of Network-based Authentication

\section{Benefits}

Users can potentially spend up to $\$ 1,000$ to purchase multimedia software and hardware without valid business justification. The desire to deploy multimedia should not be driven by the desire of users to see it, but based on business justifications. By deploying multimedia where needed and when needed, spending can be minimized and procurements grouped to obtain volume discounts and site-compliant applications. 


\section{Multimedia Technology Milestones}

The following technology evaluation and pilot project milestones must be resolved to adopt the technology.

MMedia.T.1 Pilot Multimedia Enablement of Desktop Products

MMedia.T.2 Pilot Navigable Compound Documents

MMedia.T.3 Pilot Speech and Handwriting Recognition

MMedia.T.4 Pilot Applicability of Object Databases to Multimedia

MMedia.T.5 Pilot Desktop Videoconferencing 


\section{MMedia.T.1 Pilot Multimedia Enablement of Desktop Products}

Responsible Organization

IRM, MSD

Recommended Initiation Date

$1 \mathrm{Q} / 94$
Sponsoring Organization

IRM

Status

Started

\section{Anticipated Duration}

Nine months

\section{Resource Considerations}

One-half FTE to monitor and pilot technologies and track industry trends. Cooperation and participation from MSD and Training Integration. $\$ 30,000$ in equipment and software.

\section{Discussion}

Since multimedia technology is rapidly evolving and is characterized by new products with short lives, some guidance is needed for selection of technologies and products based on business needs. Pilots will limit initial applications and product selections to those which have significant benefit and adhere to site infrastructure guidelines.

\section{Action ltems}

1) Pilot voice, image, and video enablement at the desktop.

2) Determine a suite of cross-platform data types for initial deployment.

\section{Dependencies}

Office.1.2 Install Online Scanners

\section{Benefits}

Pilots in determining valid uses of multimedia within existing desktop products will assist the development of standards and identification of necessary infrastructure improvements. 


\section{MMedia.T.2 Pilot Navigable Compound Documents}

Responsible Organization

IRM, MSD

Recommended Initiation Date

$2 Q / 94$

\section{Anticipated Duration}

Nine months

\section{Resource Considerations}

One-half FTE to monitor and pilot technologies and track industry trends. Cooperation and participation from MSD. $\$ 10,000$ in equipment and software.

\section{Discussion}

The site has little experience with compound documents architectures (CDA) and their ability to provide unified access to multiple data types within the document paradigm. A draft DOE order will require the use of SGML compound document formats for transmission of data to DOE document repositories by 1995 . The site needs to determine the owner organization for compound documents and pilot the technologies in anticipation of DMS projects and compliance with DOE requirements.

\section{Action Items}

1) Identify CDA to pilot and data types to include.

2) Conduct pilot and make recommendations.

3) Demonstrate technology to affected organizations.

4) Participate on DOE SGML teams.

\section{Dependencies}

MMedia.I.1

Develop Multimedia Principles

MMedia.I.2

\section{Benefits}

Experience gained in a pilot of compound documents will reveal the capabilities and limitations for dealing with data other than text within such a paradigm. It will identify infrastructure support issues and potential for object database applications, and educate the participants in the activities of standards bodies. 


\section{MMedia.T.3 Pilot Speech and Handwriting Recognition}

Responsible Organization

[RM

Recommended Initiation Date

$2 Q / 95$
Sponsoring Organization

IRM

Status

Not started

\section{Anticipated Duration}

Six months

\section{Resource Considerations}

One-quarter FTE; $\$ 10,000$ in equipment and software.

\section{Discussion}

Speech and handwriting recognition are highly publicized technologies that need exploration as to business applicability. The use of spoken commands could simplify user interfaces, and handwriting recognition could translate handwritten data, such as research notes, into computer-stored text data. Niche applications exist in security, authentication, and computing for the physically challenged.

\section{Action items}

1) Conduct pilot for speech recognition.

2) Conduct pilot for handwriting recognition.

3) Determine whether business case exists for use of piloted technologies.

\section{Dependencies}

MMedia.I.2

Adopt Cross-platform Fonts and Image Formats

MMedia.I.4

Enable Routine Capture and Playback of Cross-platform Audio

\section{Benefits}

Recognition technologies can provide alternative methods for data entry or program commands improving end-user productivity. 


\section{MMedia.T.4 Pilot Applicability of Object Databases to Multimedia}

\section{Responsible Organization}

IRM

Recommended Initiation Date

$3 \mathrm{Q} / 95$

\section{Sponsoring Organization}

IRM

\section{Status}

Not started

\section{Anticipated Duration}

12 months

\section{Resource Considerations}

Coordination of multimedia requirements into design of object strategies.

\section{Discussion}

Multimedia applications by nature will not be self-contained but will refer to varying types of data in multiple locations. Object technology could provide the tools for collection and management of multimedia assets.

\section{Action Items}

1) Create the object model for multimedia.

2) Pilot linkages between object database and desktop products.

3) Make recommendations regarding future use of object technology.

\section{Dependencies}

Object.C.2

Develop Site Expertise in Object-oriented Technology

\section{Benefits}

Multimedia data should be treated as mainstream data, not a special case. Its inclusion in the data and object business models is essential. 


\section{MMedia.T.5 Pilot Desktop Videoconferencing}

Responsible Organization

IRM

Recommended Initiation Date

1Q/95

\section{Sponsoring Organization}

IRM

Status

Not started

\section{Anticipated Duration}

Six months

\section{Resource Considerations}

$\$ 6,000$ in hardware and software.

\section{Discussion}

Desktop Videoconference is the first potential application to require a fully-integrated desktop multimedia workstation. The pilot will establish the technical viability for widespread implementation at SRS.

\section{Action ltems}

1) Determine available and compatible products.

2) Examine integration issues into existing infrastructure.

\section{Dependencies}

Networks.I.13 Provide Access Between SRSnet and Offsite Locations

\section{Benefits}

If desktop video can be deployed for under $\$ 250$ per workstation and tie-in is made to commercial videoconference systems, the site can save millions in travel costs. Even for site-only videoconferencing, significant savings can be achieved in reduced travel time by employees across the site. 


\section{Electronic Office Roadmap}

\section{Introduction and Destination}

To define the destination for this roadmap the following typical workday scenario is used:

You arrive at your office in the morning and $\log$ in to the network. The first thing that you do is check your electronic organizer to see what your schedule is for the day. You see that your day is open with the exception of one meeting at 4:00 PM with Dave Smith. You do not remember what the meeting is about, so you select the meeting time in your electronic calendar. Your electronic notepad opens up, and you see the purpose of the meeting as well as a preliminary agenda and list of invitees which Dave distributed.

Next you request your electronic To Do list and see your list of action items for today. You notice that along with today's action items, there are three items on the list which have automatically been carried forward from yesterday because you did not complete them. You decide to get to work on your action items after you check your mail.

You select your Electronic Inbox and a window pops up, listing five e-mail messages and describing who they are from, what they are about, and approximately how long each is. The window also lists seven voice-mail messages and three incoming faxes with date, time, and sender's name and location. Some messages of each type (e-mail, voice-mail, and fax) have already been automatically deleted, stored, forwarded, or replied to based on your personal business rules and recognition of the subject matter, originator, and/or content.

As you are scanning the subject matter of your e-mail messages, you see that one is from Dave Smith and concerns this afternoon's meeting, so you decide to read it first. Dave tells you that something has come up and he needs to reschedule the meeting for next Monday at 4:00 PM. You accept this change, and your electronic calendar is automatically updated, removing this afternoon's meeting and scheduling you for Monday afternoon's meeting. Dave is automatically notified that you have accepted the meeting change.

You don't see any other e-mail messages that you need to deal with right now, so you decide to check your voice-mail messages. Each message can be replayed through your computer speaker, earphone, or telephone handset. Your screen then presents you with several options. You can delete a message, save it, forward it, or reply to it. You request to forward a message. The system asks you if you want to append a message to it. You answer yes, and your computer or earphone beeps, signaling that it is waiting for you to record your message. (Optionally, your telephone rings, and your message is recorded until you return the handset on-hook.) After finishing your recording, you are able to address it to several people. You select the send command, and off it goes. The message is distributed to recipients using e-mail, voice-mail, and fax, as appropriate, with text-to-speech and speech-to-text processing accomplished on the fly.

You return to your Electronic Inbox and review your list of incoming faxes. You select the first one, and it is displayed on your screen. After reading it, you discard it. The second fax has some information that you need to use in a report. You copy a section of the fax image into the online Optical Character Recognition (OCR) system, and receive a text interpretation in return. You move the text into your working files storage for later inclusion in your document.

The third fax is from one of your most important customers. He has included new sketches indicating a problem which you need to deal with as soon as possible. There are three other people that you need to meet with to discuss the problem. You request your Electronic Calendar 
and tell it whom you need to meet with, for how long, what the subject of the meeting is, and where you want to meet. You also indicate that you need the department's video teleconferencing and projection equipment for the meeting. Then you request the scheduling service to find the earliest time that all four attendees, the audiovisual (AV) equipment, and the conference room are available. It finds a time at $9 \mathrm{AM}$ tomorrow, automatically schedules the $A V$ equipment and the conference room, and sends meeting notifications to the other attendees.

While you were working, a new message entered your Electronic Inbox. The subject of the message indicates that there is an electronic purchase requisition waiting for your approval. You select the new message, and an electronic form is displayed on the screen. You spend a few minutes reviewing the form, approve it electronically, and send it on its way. (Your electronic approval is binding, like any other signature. It is secure and traceable via the database transaction security and log, and a reference to your public-key cryptographic signer.) You don't know who the next person to review the form is but it doesn't matter, because the form knows where it is supposed to go next and has been correctly forwarded.

You are now ready to return to your ToDo list. The item that you choose to work on first is a report that you are writing. There are several documents which you need to reference in writing this report. You request the document retrieval service, and it provides you a menu to use for entering selection criteria. The Document Management System (DMS) uses this information to retrieve the document from the archive area. The document was stored in the DMS with embedded images. You want to include some of the diagrams in your report, so you drag and drop copies of the images into your document. You also copy text, tables, and a reference to the source document into your report before closing your work.

The next item on your To Do list is to read and comment on a report written by one of your coworkers. You check out the document from the In-Process area of the DMS and read it over. You make several suggestions by electronic markup and tagged comments. Your coworker will recognize your comments (along with those of other reviewers), and will know in what order the comments were made. When you are finished, you return possession of the document to the DMS.

Another item on your To Do list is to finish a section of a technology white paper which you are co-authoring with three other people. You retrieve the document and look over the progress that the others have made. You notice that one of the other authors is busy working on his section of the paper, but that does not matter because neither of you has write access to the other's section. You can, however, post annotations to each other's sections, even as the other writer is making updates. You notice that others have made a few comments about your previous entries, and you try to incorporate their suggestions as you continue to write. Your creative juices run dry before you finish, so you save it to come back to later.

The final item in your To Do list for the day is an item which was placed there by the work-flow management system. Your department is busy working on next year's budget requirements. Input has been received from other members of the group on training requirements for next year. You are to take that input, summarize it, and come up with the departmental training requirements. This is the last section of the budget to be done before your manager has to review and approve. Once you are finished, your manager is notified that he has an action item on the budget process.

To make the preceding scenario a reality, software and hardware for the following electronic office technologies must be available.

Client/server electronic mail

Electronic organizer

Document Management System 


\section{Roadmap to the SRS Computing Architecture}

- Shared authoring

Electronic forms routing and approval

- FAX gateway

- Electronic work flow management

- Voice-mail/e-mail integration

It should be noted that all of the items listed above are existing technologies and are available today from a variety of vendors. The goal is not to develop new technologies, but rather to implement and integrate existing technologiesinto a cohesive, highly productive electronic office. 


\title{
Starting Point
}

The core of the electronic office will be the future client/server messaging system. This system will be founded on an infrastructure of personal computers, servers, high-speed networks, distributed management and security systems, and global directory services. The state of this infrastructure is the subject of the Networked Systems roadmap.

Currently, the site electronic mail system is the host-based, multi-user ALL-IN-1 system which serves over 1,4000 users. The majority of the electronic office scenario will require a conversion over time from the existing system to a client/server system. A handful of departments onsite have already implemented group client/server mail systems; however, their connectivity to the site system is questionable at best. A team is currently working on defining a site standard for client/server mail.

A team is also now working on defining requirements for the voice-mail/e-mail integration. This project is being given additional emphasis as we look toward implementation of a sitewide voice mail system with the Replacement Telephone System which is currently being installed.

Various other limited pilots are being pursued to examine some of the technologies required for the electronic office.

\author{
Networks \\ - Network Systems \\ - Object-oriented Computing \\ - Client/Server Computing \\ Information Warehouse \\ - Multimedia Computing
}




\section{Electronic Office Infrastructure Milestones}

The following infrastructure milestones must be resolved to adopt the technology.

Office.I.1

Office.I.2

Office.I.3

Office.I.4

Office.I.5

Office.l.6

Office.I.7

Office.I.8
Implement FAX Input/Output

Install Online Scanners

Install Ubiquitous OCR

Install Client/Server E-mail

Implement Mail-enabled Applications

Implement Electronic Forms Routing and Authorization

Implement Viewers/Translators

Integrate Voice-mail and E-mail Systems 


\section{Office.l.1. Implement FAX Input/Output}

Responsible Organization

IRM

Initiation Date

$1 Q / 94$

\section{Anticipated Duration}

24 months

\section{Resource Considerations}

Funding will be required for the purchase of the network fax equipment, as well as hardware and software required to route inbound faxes.

\section{Discussion}

FAX services must be provided on the network. These services must include inbound routing to each user's individual electronic inbox, as well as outbound print-to-FAX and mail-to-FAX. Users must be able to scan paper documents into their electronic desktops and send them out over network faxes. They must also be able to easily OCR any incoming fax for inclusion into revisable electronic documents.

\section{Action Items}

1) Purchase and deploy network FAX equipment for outbound faxes.

2) Establish routing to inbound FAX recipients.

3) Enter initial list of out-bound FAX recipients in global directory.

\section{Dependencies}

Office.I.2

Install Online Scanners

Office.I.3

Install Ubiquitous OCR

Office.I.7

Implement Viewers/Translators

Office.A.1

\section{Benefits}

Many SRS employees depend on fax technology for external communications. Network fax input/output will allow those employees to process their fax communications in the same manner in which they process all other communications-from within their electronic desktop. 


\section{Office.1.2. Install Online Scanners}

\section{Responsible Organization}

IRM

Initiation Date

$1 Q / 94$
Sponsoring Organization

IRM

\section{Status}

Project in pilot

\section{Anticipated Duration}

24 months

\section{Resource Considerations}

Funding will be required to purchase scanner hardware and software.

\section{Discussion}

Because paper-based information will no longer be handled, scanners must be available (via user access or centralized service) to enter paper-based data into the electronic system. Personal/group scanners should be easily accessible for small, frequent scans, and larger scanners, should be available for batch scanning larger jobs. Easily accessible scanners are also needed to facilitate desktop fax input/output.

\section{Action ltems}

1) Evaluate requirements for personal, group, and central scanners.

2) Purchase and deploy scanners.

3) Set policy on use of scanners.

\section{Dependencies}

Netsys.I.11 Connect Office Peripherals Directly to Network

\section{Benefits}

With online scanners readily available users will be able to easily enter paper-based data into their electronic desktop. Once this data is entered electronically, it can then be stored, included in electronic documents, modified, mailed, or faxed. 


\section{Office.l.3 Install Ubiquitous OCR}

Responsible Organization

IRM

Initiation Date

$1 Q / 94$

\section{Anticipated Duration}

24 months

\section{Resource Considerations}

Six man-months.

Funding will be required to purchase OCR software.

\section{Discussion}

Because paper-based information will no longer be handled, optical character recognition (OCR) services must be available for batch and on-request conversion of scanned and faxed bitmapped images into text for editing and further routing via non-graphic transports (for example, voice-mail.) OCR software should be available on individual workstations for onrequest conversions, as well as on batch scanner/OCR stations for batch processing of larger jobs.

\section{Action ltems}

1) Purchase and deploy OCR software.

2) Train users in use of $O C R$ system.

3) Integrate OCR into automatic and manual transmission and translation processes.

\section{Dependencies}

Office.1.2

Install Online Scanners

\section{Benefits}

OCR software will work in conjunction with online scanners to provide users the capability to include paper-based or faxed data on their electronic desktops. 


\section{Office.l.4. Install Client/Server E-mail}

Responsible Organization

IRM

Initiation Date

$1 \mathrm{Q} / 94$

\section{Anticipated Duration}

36 months

\section{Resource Considerations}

Nine man-years.

Funding will be required to purchase client/server e-mail client software, as well as server hardware and software.

\section{Discussion}

To enable an electronic (paperless) office, true client/server (C/S) e-mail with specific mailenabled applications must be provided on the SRS network system. C/S e-mail must provide rules which are processed on the server for automatic message translation, forwarding, and reply. The mail system will be the backbone of the electronic desktop. All communications, including e-mail, fax, voice-mail, and eventually video-mail messages, should be received through the electronic inbox. The $\mathrm{C} / \mathrm{S}$ e-mail system must interface with mail-enabled applications such as group scheduling, voice-mail integration, electronic routing and approval, word-processing, spreadsheets, etc.

\section{Action Items}

1) Select a site-standard $\mathrm{C} / \mathrm{S}$ e-mail.

2) Design $C / S$ mail infrastructure.

3) Formulate installation and migration plans.

4) Purchase and install the $C / S$ mail system software.

5) Train users on use of $C / S$ mail.

6) Migrate accounts from ALL-IN-1 to C/S e-mail.

\section{Dependencies}

Netsys.I.3 .

Netsys.I.4

Netsys.I.5

Netsys.I.7

Netsys.I.11

Office.I.1
Ensure Broad Availability of Networked Workstations

Migrate to New NOS

Initiate NOS Global Directory

Complete Installation of Territorial File Servers

Connect Office Peripherals Directly to Network

Implement FAX Input/Output 
Office.I.7

Implement Viewers/Translators

Office.A.1

Implement Security Policy and Procedures

\section{Benefits}

Electronic mail usage at SRS is already pervasive, with approximately 15,000 current subscribers. In addition to traditional text-based electronic mail, which is becoming increasingly important as a communications mechanism, the client/server e-mail system will provide users with a single electronic inbox for all electronic communications. Also, applications are now relying on electronic mail as a mechanism for notification and information distribution as they become mail-enabled. In addition to providing the infrastructure for electronic communications and mail-enabled applications, the client/server e-mail products provide enhanced mail processing capabilities. These capabilities include automatic rules-based filtering of messages, improved user interfaces through GUI clients, and multimedia support. 


\section{Office.l.5. Implement Mail-enabled Applications}

Responsible Organization

IRM

Initiation Date

$4 Q / 94$
Sponsoring Organization

IRM

\section{Status}

In pilot

\section{Anticipated Duration}

30 months

\section{Resource Considerations}

One man-year.

\section{Discussion}

One of the major benefits of the client/server mail system is its use as a notification and distribution mechanism for various applications. To take full advantage of the $\mathrm{C} / \mathrm{S}$ mail system, applications must be able to transparently use it for distributions. Examples include ability to send a word-processing document, spreadsheet, electronic form, or electronic meeting request without ever leaving the applications in which they are created.

\section{Action Hems}

1) Determine which existing applications need to be mail-enabled.

2) Determine which mail-enabled applications to implement.

3) Purchase and deploy mail-enabled applications.

4) Train users in use of functionality.

\section{Dependencies}

Office.I.4

Install Client/Server E-mail

Office.A.1

Implement Security Policy and Procedures

\section{Benefits}

Implementation of mail-enabled applications will simplify many tasks which are now cumbersome and time consuming. 


\section{Office.I.6. Implement Electronic Forms Routing and Authorization}

Responsible Organization

IRM

Initiation Date

$3 Q / 94$

\section{Anticipated Duration}

30 months

\section{Resource Considerations}

Six man-months.

Funding will be required to purchase routing and authorization software.

\section{Discussion}

An electronic forms routing and authorization system is needed to simplify and speed up the processing of existing paper forms. This system should be integrated with the site client/server e-mail system so that forms needing to be processed can be accessed directly from a user's electronic inbox.

\section{Action ltems}

1) Formulate electronic forms routing and authorization system infrastructure design.

2) Implement forms routing pilot.

3) Purchase and deploy electronic forms routing and authorization system.

3) Train users in use of new system.

\section{Dependencies}

Office.I.4

Install Client/Server E-mail

Office.A.1

Implement Security Policy and Procedures

Office.A.3

Migrate Forms

Office.A.5

Recognize Electronic Signatures as Binding

\section{Benefits}

The use of electronic forms, as well as the electronic routing and authorization of those forms, will simplify and speed up the processing and approval of existing paper forms. In addition, data fields from electronic forms can be captured and stored for use in other applications and reports. For example, the amount due an employee on a travel expense report could be captured after all electronic approvals are complete. A check could then be automatically cut and sent to the employee. 


\section{Office.l.7. Implement Viewers/Translators}

\section{Responsible Organization}

IRM

Initiation Date

$3 Q / 94$

\section{Anticipated Duration}

18 months

\section{Resource Considerations}

Six man-months. Funding will be required to acquire viewer/translator software.

\section{Discussion}

A variety of data formats will continue to play a part in the electronic office. Data formats will be translated into a form usable by the workstation. The translation should be transparent to the user. The following list serves as an example of the desired translators and viewers:

- Standard archiving format for multiple data forms (for example, PDF)

- Standard text viewer (for example, SGML)

- Standard table viewer

- Standard graphic viewer

- Standard full-motion and sound video viewer (for example, Quicktime)

- Standard sound player

- Word processing translators

- Spreadsheet translators

- Database translators

- Graphic translators (including presentation package reformators)

- Sound translators

- Video translators

Compression translators

\section{Action Items}

1) Ensure required translators are available.

2) Ensure required viewers are available.

3) Acquire/implement a translation server or automatic service. 


\section{Roadmap to the SRS Computing Architecture}

\section{Dependencies}

Netsys.I.1 Install Robust Client Workstations

\section{Benefits}

With consistent data viewers and translators in place, users will be able to view any type of data which may be sent to them. 


\section{Office.1.8. Integrate Voice-mail and E-mail Systems}

Responsible Organization

IRM

Initiation Date

$1 \mathrm{Q} / 94$

\section{Sponsoring Organization}

IRM

\section{Status}

Working group started

\section{Anticipated Duration}

36 months

\section{Resource Considerations}

36 man-months. Funding will be required to purchase voice/electronic mail gateway.

\section{Discussion}

The electronic office will also be what has been referred to as a spartan office; that is, only one communication device is required in the office. That communication device will be capable of placing and supporting voice-communication (telephone) calls, and will eventually handle desktop videoconferencing, as required. With this technology in place, integration of message methods, voice-mail and e-mail, will be accomplished. The integration will occur in several phases:

\section{Action Items}

1) Establish notification of voice-mail messages in e-mail.

2) Achieve management (delete, listen to via phone, file, forward, reply) of voice-mail messages via e-mail interface.

3) Integrate call management functions (dial, hold, conference, forward) into computer.

4) Integrate phone audio functions (speak, listen, voice-message) into computer.

5) Integrate e-mail and voice-mail backend into single messaging database.

6) Integrate e-mail and voice-mail directories, and include $x .500$, Internet, and phone-service provider directory lookups.

\section{Dependencies}

Networks.I.1

Install the Replacement Telephone System

MMedia.I.5

Implement Storage, Multimedia Servers, CD-ROM, and Improved Monitors

MMedia.I.4

MMedia.A.1

Office.I.4

Enable Routine Capture and Playback of Cross-platform Audio

Office.I.5

Approve Use of Multimedia Data Types

Install Client/Server E-mail

Implement Mail-enabled Applications

Office.A.1

Implement Security Policy and Procedures 


\section{Benefits}

Once the voice-mail and e-mail systems are integrated, all communications will be handled through a single device. Productivity will be improved as users will be able to quickly scan and process all types of communication in a consistent format. 


\section{Electronic Office Administrative Milestones}

The following administrative milestones must be resolved to adopt the technology. Each is described in more detail in the following sections.

Office.A.1 Implement Security Policy and Procedures

Office.A.2

Scan in Historical and Reference Data

Office.A.3

Migrate Forms

Office.A.4

Implement Online Librarian and Document Repository

Office.A.5

Recognize Electronic Signatures as Binding

Office.A.6

Implement the Paperless Office 


\section{Office.A.1 Implement Security Policy and Procedures}

Responsible Organization

Computer Security

Initiation Date

$3 Q / 94$

\section{Anticipated Duration}

12 Months

\section{Resource Considerations}

One man-year.

\section{Discussion}

New security policies, procedures, and supporting technologies must be developed to accommodate information workers without paper. Policies must be established to accommodate the trend to portable computing by allowing remote access and defining requirements for data on portable machines. Also necessary will be policies allowing for electronic distribution of data and electronic authorization and signatures.

\section{Action ltems}

1) Establish policy and procedures to allow remote access.

2) Establish policy and procedure for portable data.

3) Establish policy and procedure for data interchange and distribution.

\section{Dependencies}

None

\section{Benefits}

These new policies will allow implementation of the electronic office from the security point of view. The users will be more productive because they will be able to use their portable equipment while away from the office. Also, the current flood of paper forms will be reduced by migrating to electronic forms and authorizations. 


\section{Office.A.? Scan in Historical and Reference Data}

Responsible Organization

MSD

Initiation Date

$1 Q / 95$

\section{Anticipated Duration}

36 months

\section{Resource Considerations}

Nine man-years.

\section{Discussion}

Some historical and reference data exists only in paper form at present, but must continue to be readily available and referenced by information workers in the electronic office. That data must be identified and scanned into electronic format. Indexing and/or OCR should be accomplished for each document.

This should be coordinated with ongoing site DMS scanning efforts.

\section{Action Items}

1) Identify data for scanning.

2) Batch scan and OCR documents.

3) Index documents and place in data repository.

\section{Dependencies}

Office.I.2

Install Online Scanners

Office.I.3

Install Ubiquitous $O C R$

Office.I.7

Implement Viewers/Translators

Office.A.4

Implement Online Librarian and Document Repository

Office.P.1

\section{Benefits}

Information workers will have easy access historical and reference data, saving time and money when searching for needed data. 


\section{Office.A.3 Migrate Forms}

Responsible Organization

MSD

Initiation Date

$1 Q / 94$

Anticipated Duration

24 months

\section{Resource Considerations}

Nine man-years.

\section{Discussion}

SRS must disband the use of single- and multi-part paper forms in favor of electronic forms with mail-enabled electronic routing and approval. Once an electronic forms routing and approval system is in place, existing paper forms must be migrated to electronic format.

\section{Action Items}

1) Determine what existing forms to carry forward into the electronic office.

2) Determine which forms require visual replication on screen, and which forms need to be reformatted so as to be better suited for electronic presentation.

3) Rebuild the forms and the backend data structures.

4) Build and initiate (electronically) business rules for forms processing.

5) Deploy forms and train users.

\section{Dependencies}

Office.I.4

Install Client/Server E-mail

Office.I.5

Office.A.5
Implement Mail-enabled Applications

Recognize Electronic Signatures as Binding

\section{Benefits}

The use of electronic forms, as well as the electronic routing and approval of those forms, will simplify and speed up the processing and approval of existing paper forms. 


\section{Office.A.4 Implement Online Librarian and Document Repository}

Responsible Organization

IRM

Initiation Date

$1 Q / 95$

\section{Anticipated Duration}

24 months

\section{Resource Considerations}

Two man-years.

\section{Discussion}

Not only must the electronic office have online access to historical and other references as well as current operational data, but a document management function, in the form of online library administration, must also be provided. Processes of this librarian include user submission and indexing, administrator acceptance and cataloging, user research, and administrative archiving to cheaper, long-term storage.

This is to be coordinated with ongoing site DMS effort.

\section{Action ltems}

1) Configure online document repository.

2) Establish policies for online document repository.

3) Identify and train supportive online document repository administrative resources.

4) Train users on submission and access for research and reference in online document repository.

\section{Dependencies}

Office.A.2

Scan in Historical and Reference Data

Office.I.7

$$
\text { Implement Viewers/Translators }
$$

\section{Benefits}

Easier access to, along with better organization and control of electronic documents. 


\section{Office.A.5 Recognize Electronic Signatures as Binding}

Responsible Organization

Site Legal

Initiation Date

$1 \mathrm{Q} / 95$
Sponsoring Organization

IRM

Status

Not started

\section{Anticipated Duration}

12 months

\section{Resource Considerations}

Three man-months.

\section{Discussion}

The secure electronic signature must be formally recognized as an ethically and contractually binding authorization. Electronic forms routing and authorization, a major component of the electronic office, cannot be implemented without this.

\section{Action Items}

1) Formalize policy on electronic signatures.

2) Coordinate electronic signature policy with all internal and external customers and suppliers.

3) Select technology for electronic signatures.

4) Deploy, integrate, and utilize electronic signature technology.

\section{Dependencies}

None

\section{Benefits}

Electronic forms may be routed and approved without ever having to go to paper form. This will greatly reduce the time and effort required for forms to be processed. 


\section{Office.A.6 Implement the Paperless Office}

Responsible Organization

IRM

Initiation Date

$1 \mathrm{Q} / 96$

Anticipated Duration

12 months

\section{Resource Considerations}

One man-year.

\section{Discussion}

To fully implement the electronic office and effectively migrate to it, the use and distribution of paper must be analyzed and reduced for electronic office locations. While users will ideally recognize the benefits of the electronic office and voluntarily move toward it, it is expected that the electronic office will be fully implemented only after management policies requiring it are put in place.

\section{Action ltems}

1) Develop policy on use of electronic documents and forms.

2) Establish procedures for reducing dependency on paper forms and documents.

3) Implement electronic document and forms policy.

\section{Dependencies}

Office.I.1

Office.I.2

Office.I.3

Office.I.4

Office.I.5

Office.I.6

Office.I.7

Office.I.8

Office.A.2

Office.P.1 -

Office.P.2

\author{
Implement FAX Input/Output \\ Install Online Scanners \\ Install Ubiquitous OCR \\ Install Client/Server E-mail \\ Implement Mail-enabled Applications \\ Implement Electronic Forms Routing and Authorization \\ Implement Viewers/Translators \\ Integrate Voice-mail and E-mail Systems \\ Scan in Historical and Reference Data \\ Implement Document Management System \\ Implement Electronic Data Interchange
}

\section{Benefits}

The implementation of the paperless office will result in conservation of paper supplies, more efficient work by office workers, easier access to electronic resources, and traceability of work and data. 


\section{Electronic Office Process Milestones}

The following work flow milestones must be resolved to adopt the technology. Each is described in detail in the following sections.

Office.P.1

Implement Document Management System

Office.P.2

Implement Electronic Data Interchange 


\section{Office.P.1 Implement Document Management System}

Responsible Organization

IRM

Initiation Date

$1 \mathrm{Q} / 94$

\section{Anticipated Duration}

24 months

\section{Resource Considerations}

Funding will be required to acquire the Document Management System hardware and software.

\section{Discussion}

The electronic office will depend heavily on a fully capable and integrated document management system. The system will provide for group authoring with multiple access, section check-out and -in, security and access control, indexing, index and full-text search, and archiving. The system will be administered by automatic and manual librarian functions.

\section{Action ltems}

1) Determine DMS functional specifications.

2) Acquire DMS components.

3) Establish DMS policies and procedures (business rules).

4) Implement DMS.

\section{Dependencies}

Office.I.4

Office.A.2

Office.A.3

Office.A.4

Neysys.I.1

\section{Benefits}

Group authoring. Better control of and access to personal and group documents
Install Client/Server E-mail

Scan in Historical and Reference Data

Migrate Forms

Implement Online Librarian and Document Repository

Install Robust Client Workstations
Sponsoring Organization

MSD

Status

Ongoing program to establish DMS 


\section{Office.P.2 Electronic Data Interchange}

Responsible Organization

Contracting

Initiation Date

$3 Q / 96$

\section{Anticipated Duration}

18 months

\section{Resource Considerations}

12 man-months.

\section{Discussion}

Begin transactions and data deliveries and receipts from/to internal and external customers and suppliers via electronic routing and distribution using electronic data interchange (EDI) standards.

\section{Action ltems}

1) Establish working agreements with all internal and external customers and suppliers to transact business and reporting electronically.

2) Develop EDI communications capability with gateways to suppliers.

\section{Dependencies}

\section{Office.I.1}

Office.I.4

Implement FAX Input/Output

Office.A.1

Office.A.3

Office.A.5

\section{Sponsoring Organization}

IRM

\section{Status}

Not started

\section{Benefits}

Faster, more consistent communication with customers and suppliers. Quicker turnaround on communications with suppliers. 


\section{Electronic Office Technology Milestones}

The following technology milestone must be resolved to adopt the technology. Each is described in more detail in the following sections.

Office.T.1 Implement Voice Processing 


\section{Office.T.1 Implement Voice Processing}

Responsible Organization

IRM

Initiation Date

1Q/97

\section{Sponsoring Organization}

IRM

\section{Status}

Not started

\section{Anticipated Duration}

48 months

\section{Resource Considerations}

Two man-years.

\section{Discussion}

Current voice-processing capability on standard office workstations includes simple voice recording and playback; unreliable, user-specific (trained) voice command; and rudimentary text-to-voice simulation. The following action items represent SRS incorporation of coming technology.

\section{Action ltems}

1) Incorporate high-speed digital signal-processing engines into all workstations capable of video and voice processing.

2) Acquire artificial intelligence voice command processing software for workstations.

3) Build initial voice-command functionality and deploy to all workstations.

4) Acquire real-time, speaker-independent voice recognition software for all workstations.

\section{Dependencies}

MMedia.A.1

Approve Use of Multimedia Data Types

\section{Benefits}

Hands-off computer interface and voice-to-text processing (dictation). 


\section{Object-oriented Technology}

\section{Introduction and Destination}

All site computing resources-desktop workstations, servers, and mainframes-exist as nodes on a unified site network that can conceptually be thought of as the site computer. Data is wellmodeled, managed, and located on the most appropriate platform for its intended use. Applications as they are known today do not exist. Instead, there are libraries of well-defined objects that conform to accepted object technology concepts and structures. These objects are interconnectable and re-usable and can be combined as desired to create functionality.

Application development, then, is more focused on creating unique combinations of objects than on implementation of logic. Business processes and functions execute in a client/server environment such that data, processing logic, and user interfaces are distributed and implemented over the network at the most appropriate computer node for that specific activity.

A technical support function provides administration of the object libraries analogous to that for DBMSs. A technical object development and maintenance function is responsible for developing new objects and maintaining and revising existing objects. Well documented and enforced procedures and standards exist to administer these activities.

Instances of objects are stored within commercially available DBMSs that are extensions of traditional RDBMSs.

The site computer development staffs have all been well trained in the concepts of object technology and in the use of objects to provide end user functionality. The site system development and project management methodologies are built around object technology. The application development staff routinely employs object technology in their work. All programming to enable the use of objects is performed using the site standard programming language. Objects are purchased where commercially available. They are revised and extended by the site object technical staff as necessary to accomplish the desired purpose. All site purchased software is object-based and compatible with site object standards.

Legacy systems-the set of applications that have evolved over time to run the business and which embody a wide spectrum of past technologies and business expediencies - continue to provide essential business functionality in the object-oriented environment for as long as economically and functionally justified. Technologies and functionalities embedded in legacy systems are continually reviewed and analyzed for the purpose of replacing them with objectedoriented $(\mathrm{OO})$ equivalents when technically and economically feasible. However, the reality of the long-term existence of legacy systems in the future is recognized, and the object-oriented environment is structured and developed to ensure coexistence with legacy systems. Initiatives are supported to enable the gradual evolution and migration of legacy systems to a completely object environment in a cost-effective manner. No computer development initiatives are implemented outside of the object environment unless shown to be in the site's best interest. 


\section{Starting Point}

The site has a solid (and improving) network foundation. Additionally, the growth of WISDOM will provide a framework for centrally managed, distributed services to end users. Planned improvements to the site desktop will provide the increased computing capability required to handle the demands of object-oriented technology. In other words, the hardware infrastructure necessary to support objects is now or soon will be in place.

The software side of the object picture is a different story. The first major issue is a lack of general understanding of the technology within site computing organizations. What understanding exists is, for the most part, cursory in nature. Creating a core of object professionals will require a commitment to provide training and develop a strong mentoring program.

One of the prime motivations for moving to object technology is to broker core code to many applications in the same way that DBMSs broker data to many applications. There exist a great many functional objects (window, button, menu, database access, video, audio, etc.) which can be purchased for this use. Unfortunately, the data objects (employee, facility, document) which would provide the most value to site developers are not available and must be custom built. The site must make the investment in terms of skilled developers to create data objects that reflect the site business. An organization must be chartered to create such a Site Object Framework (SOF).

The site computer development staffs have all been well trained in the concepts of procedural technology. The site system development and project management methodologies are built around procedural technology. Most core systems development is performed using a proprietary programming language. Core business systems are seldom purchased, although this is changing. Those that are purchased are usually extended to the point that the site is unable to incorporate future releases.

Legacy systems, those applications that have evolved over time to run the business, are continually patched and repatched to meet changing customer requirements, even when these changing requirements are inconsistent with the original design criteria of the application. As these systems degenerate from a pure design, modifications to the systems become increasingly difficult. Eventually, the lack of encapsulation of procedural systems will cause ripple effects when seemingly innocuous changes present serious challenges to the application maintainer. As a result, core application maintenance is increasingly constrained by it own efforts.

There is currently no significant presence of object development tools here at the site. Requirements and specifications for the tools must be established and worked through the purchasing system. 


\section{Object-oriented Infrastructure Milestones}

The following computing infrastructure milestones must be resolved to adopt the technology.

Object.I.1

Establish Site Object Framework Team (SOFT)

Object.I.2

Adopt Site Object Messaging Standard

Object.I.3

Establish Object Framework

Object.I.4

Determine and Implement the Necessary Changes to WISDOM to Support an Object-oriented Environment

Object.I.5

Re-engineer Existing Core Applications as Object Applications 


\section{Object.l.1 Establish Site Object Framework Team (SOFT)}

Responsible Organization

IRM

Recommended Initiation Date

$3 Q / 94$

\section{Anticipated Duration}

24 months

\section{Resource Considerations}

The SOFT will be composed of a few (4-7) full-time, highly talented software developers and business process and technical analysts, with significant augmentation by business area experts for short duration as needed.

\section{Discussion}

The goal of the SOFT is to develop a hierarchy of business and technical objects that reflect the mission of the site. Examples of these objects might be "Facility" and "Employee," concepts which exist in virtually every piece of software at the site. By creating robust implementation of these concepts as objects, software which utilizes these concepts can be rapidly constructed and easily maintained. In other words, the purpose of the SOFT is to develop the site object library in conjunction with the end users of those objects.

\section{Action Items}

1) Establish SOFT as an SRS entity (probably under ALD).

2) Select a prototyping tool.

3) Analyze existing site software and databases to find candidate objects.

4) Work candidate objects into a coherent framework and test the framework by prototyping existing site software utilizing the framework.

5) Repeat items 3 and 4 until the framework stabilizes.

\section{Dependencies}

None

\section{Benefits}

Provides a central organization responsible for the development, maintenance, and extension of the site object framework. 


\section{Object.I.2 Adopt Site Object Messaging Standard}

Responsible Organization

IRM

Recommended Initiation Date

$3 Q / 94$
Sponsoring Organization

IRM

Status

None

\section{Anticipated Duration}

Six months

\section{Resource Considerations}

Will require the creation of a short-term team of technical personnel with an appreciation of objects. The team should be as small as is consistent with gaining consensus among impacted site development and network groups.

\section{Discussion}

Objects communicate via messages. Several different standards are emerging in this area (for example, Sun's DOE, HP and NeXT's PDO, and others). The Object Management Group's CORBA standard is an effort to create a uniform object messaging standard, and most major players have agreed to support this common standard. This support, however, is likely to be at the same level as that at which the industry supports SQL. That is, each vendor supports a minimal set of constructs, while providing most desirable features in proprietary extensions to the basic standard, thus limiting interoperability among objects from competing vendors.

\section{Action Items}

1) Form team.

2) Examine available options to determine salient features.

3) Recommend standard.

4) Gain approval as site-standard.

\section{Dependencies}

None

\section{Benefits}

An object messaging standard allows objects from different hierarchies to communicate with one another. 


\section{Object.I.3 Establish Object Framework}

Responsible Organization

IRM

Recommended Initiation Date

$1 \mathrm{Q} / 95$

Anticipated Duration

24 months
Sponsoring Organization

SITE

Status

None

\section{Resource Considerations}

This is one of the key stumbling blocks to an object implementation. This Site Object Framework will require the continued effort of a number (4-8) of the site's most talented developers.

\section{Discussion}

The Object Framework is a set of object classes arranged in a hierarchical structure. This set of classes is reused and extended by application developers to construct applications. Functional (domain-independent) objects can be obtained commercially and will form the basis of the object framework. Data (domain-dependent) objects, such as "Employee," "Part," and "Meeting," are necessarily business-specific and will have to be specially developed to incorporate the site requirements for these representations.

\section{Action ltems}

1) Design and implement a trial object framework.

2) Test Site Object Framework by construction of a robust pilot core application (repeat design step as necessary).

3) Release Site Object Framework.

4) Incorporate generalizations of appropriate application-specific code into site object framework.

5) Extend Site Object Framework in new areas to further empower developers.

\section{Dependencies}

Object.I.1 Establish Site Object Framework Team (SOFT)

\section{Benefits}

The Site Object Framework provides a core of functionality which can be easily used and extended to meet site application requirements, providing higher quality applications in a much more responsive time frame. 


\section{Object.I.4 Determine and Implement the Necessary Changes to WISDOM to Support an Object-oriented Environment}

Responsible Organization

IRM

Recommended Initiation Date

$4 Q / 94$
Sponsoring Organization

IRM

Status

None

\section{Anticipated Duration}

Six months

\section{Resource Considerations}

This should be a joint effort between the SOFT, the organization responsible for WISDOM maintenance, and those responsible for WISDOM evolution. This is a relatively small effort involving, at most, 3 man-months.

\section{Discussion}

At this point, WISDOM appears to be the most likely vehicle for the client/server, workstationcentric applications called for by the site computing architecture. As such, it is imperative that WISDOM be extended to support object-oriented applications. In large part, the modifications to WISDOM will be minor. No major changes to the WISDOM framework are anticipated, but certain products will have to be added to facilitate objects, particularly in terms of remote messaging between objects.

Action ltems

1) Form team.

2) Determine object impact on WISDOM.

3) Recommend WISDOM modifications to support objects.

4) Implement modifications.

\section{Dependencies}

Object.I.1

Establish Site Object Framework Team (SOFT)

Netsys.I.1

Install Robust Client Workstations

\section{Benefits}

Extends WISDOM to serve as the distribution and management vehicle for local and remote objects. 


\section{Object.I.5 Re-engineer Existing Core Applications as Object Applications}

Responsible Organization

IRM

Recommended Initiation Date

$4 Q / 96$

\author{
Sponsoring Organization
}

IRM

Status

None

\section{Anticipated Duration}

36 months

\section{Resource Considerations}

This will obviously consume a significant percentage of application development resources for the duration of the re-engineering project(s).

\section{Discussion}

It is certainly not necessary to re-implement all site core applications as $\mathrm{OO}$ applications. Whether or not a particular application should be re-engineered is a function of the maintenance costs, usability, and integration needs of the application in question. An application should be re-engineered when the frustration level of the end users and the maintenance costs of the existing system exceed equilibrium with the development costs using an increasingly robust object framework.

\section{Action Items}

1) Re-implement selected site core systems as $O O$ systems as per respective object migration plans.

\section{Dependencies}

Object.I.3

Object.C.2

Object.T.1

oriented Envi

\section{Benefits}

Re-engineering selected site applications as $\mathrm{OO}$ applications should reduce maintenance costs for these applications, increase functionality of these applications, provide for enhanced interoperability between applications, and significantly improve the user interface of these applications. 


\section{Roadmap to the SRS Computing Architecture}

\section{Object-oriented Administrative Milestones}

The following administrative milestones must be resolved to adopt the technology.

Object.A.1

Object.A.2

Object.A.3
Direct that Future Site Applications Should Be Object-oriented

Procure Object-oriented Technology

Develop Object Migration Plans for Existing Systems 


\section{Object.A.1 Direct that Future Site Applications Should Be Object- oriented}

Responsible Organization

IRM

Recommended Initiation Date

$3 Q / 94$

\section{Sponsoring Organization}

Site

Status

None

\section{Anticipated Duration}

12 months

\section{Resource Considerations}

This is the process of achieving consensus among high-level management that $O O$ technology is to be pursued as the technical direction of choice. This will require four to six man-months of technical effort to prepare a proposed $O O$ strategy and sell that strategy to management. Management effort will also be required to sell this strategy to higher levels of management. This will require a champion for objects, willing to devote a significant effort (10\% - 20\%) for the duration of this process. Senior level management will also be impacted in coordinating responses to any proposed strategy. While the actual amount of effort required for this step is fairly low, it requires the expenditure of effort at fairly high levels.

\section{Discussion}

An activity as broad as object migration should not be undertaken without an implementation. This document forms a first step in that direction. An effort of this type requires active high level management support in order to succeed. Resistance to change is natural, and objectoriented technology requires substantial amount of change. Without strong support (bordering on mandate), migration to objects will not succeed.

\section{Action Items}

1) Education of IRM, Computer Council, general Site, and DOE in the Benefits of Objects

2) Development of a Straw Man Object Policy for Approval by the Site

3) High-level approval of object-oriented technology as the Site default standard

\section{Dependencies}

None

Benefits .

Object-oriented technology has its price in terms of infrastructure enhancement and developer training, but this cost is far outweighed by a development time that is three to six times faster than conventional technology, increased application flexibility and reliability, decreased program maintenance costs, increased application interoperability, and improved user interface. 


\section{Object.A.2 Procure Object-oriented Technology}

Responsible Organization

IRM

Recommended Initiation Date

$1 Q / 95$

\section{Sponsoring Organization}

IRM

Status

None

\section{Anticipated Duration}

18 months

\section{Resource Considerations}

The difficulty of a major software acquisition is well known to most of the site. This initial specification phase will require four to six man-months to develop. This activity will be hampered by a shortage of trained object developers at the site. Purchasing will be required to commit a significant percentage of a buyer (two man-months) over the duration of the acquisition process. Installation of the tool should be fairly straightforward (one man-month).

\section{Discussion}

Object-oriented technology is going to require substantial changes in the developer toolset. Acquisition of these tools is going to require a major effort.

\section{Action Items}

1) Develop specifications for object-oriented development tool.

2) Purchase tool.

3) Install tool.

\section{Dependencies}

Object.A.1

Direct that Future Site Applications Should Be Object-oriented

\section{Benefits}

While OO technology is, in theory, applicable to any development environment, practical considerations require that a commercial tool be acquired to facilitate the use of objects. Successful conclusion of this milestone provides the site with such a tool. 


\section{Object.A.3 Develop Object Migration Plans for Existing Systems}

\section{Responsible Organization}

IRM

Recommended Initiation Date

$3 \mathrm{Q} / 95$

\section{Sponsoring Organization}

IRM

Status

None

\section{Anticipated Duration}

18 months

\section{Resource Considerations}

The merits of migrating a particular application to objects must be considered on a case-by-case basis. The effort required to develop a migration plan for a particular system depends upon the complexity of the system and the type of migration desired. Generally speaking, the development of a migration plan will require one-man month (simplest case) to 12 man-months (worst case) for each system.

\section{Discussion}

Each core business system developed and/or maintained by IRM should be considered for possible migration to objects. Possible types of migration (in increasing order of effort and longterm desirability) include the following:

1) None. Use the system as is.

2) Replace the 3270 front-end with an object front-end (middleware).

3) Port the existing application directly to $O O$ technology using commercial/SOF objects where possible and developing application-specific objects where necessary.

4) Port the existing application directly to $O O$ technology using commercial/SOF objects where possible and extending the SOF where necessary.

5) Redoing the existing application from the requirements on up, using commercial/SOF objects where possible and developing application specific objects where necessary.

6) Redoing the existing application from the requirements on up, using commercial/SOF objects where possible and extending the SOF where necessary.

\section{Action Items}

1) Manager responsible for each system develops migration plan.

2) Plans reviewed by IRM management to determine priority of reimplementation. 


\section{Dependencies}

Object.I.3 Establish Object Framework

\section{Benefits}

Just as it is desirable to enhance the interoperability of new systems using object technology, it is desirable to enhance the interoperability between existing systems with new systems (or with other existing systems). Some mechanism must exist to encourage the migration of these legacy systems to objects over a reasonable time frame. 


\section{Object-oriented Cultural Milestones}

The following cultural milestones must be resolved to adopt the technology. Each milestone is discussed in more detail in the following sections.

Object.C.1

Implement Matrix Project Team Approach

Object.C.2

Develop Site Expertise in Object-oriented Technology

Object.C.3

Establish Reward System for Code Reuse 


\section{Object.C.1 Implement Matrix Project Team Approach}

\section{Responsible Organization}

IRM

Recommended Initiation Date

$3 \mathrm{Q} / 95$
Sponsoring Organization

IRM

Status

None

\section{Anticipated Duration}

Six months

\section{Resource Considerations}

For the most part, this is being accomplished by the IRM reorganization.

\section{Discussion}

An object-oriented development environment requires that several roles be accounted for during application development (some roles may require several people, or one person may handle multiple roles depending on the application). These roles include the application designer, the domain expert, the application developer, the object developer, and the object architect/consultant. To pull people together to create an application, a mechanism must exist to handle the exchange of people matrixed from different organizations to perform a specific task.

\section{Action Items}

1) Organize IRM into discrete talent pools.

2) Develop/maintain applications by drawing teams from these pools.

\section{Dependencies}

None

\section{Benefits}

This milestone organizes IRM into a structure that is more conducive to object development than the current project-based organization. 


\section{Object.C.2 Develop Site Expertise In Object-oriented Technology}

\section{Responsible Organization}

IRM

Recommended Initiation Date

$1 \mathrm{Q} / 95$

\section{Sponsoring Organization}

IRM

\section{Status}

None

\section{Anticipated Duration}

12 months

\section{Resource Considerations}

Training will require a substantial effort. For the most part, site expertise in this area is at a premium, and we will probably have to go outside of WSRC to obtain trainers for this technology (at least, initially). Setting up training for all developers in IRM is not trivial (two man-months) and running all of the developers through the training requires a large effort (24 man-months). Moreover, transition to $O O$ technology is not merely learning to use a new tool, it requires a paradigm shift on the part of developers. A transition period of three to six months will exist, during which application developer productivity will fairly low. Additionally, more adept object developers will serve in a mentor role during this period.

\section{Discussion}

The single most sweeping change required by object-oriented technology is the change in focus required by application developers. This sort of shift is not effected by a week or two of training. It requires an extensive effort on the part of the development organizations to redefine the way that applications are conceived.

\section{Action ltems}

1) Rotate all developers through a course in basic object concepts.

2) Develop object experts with more rigorous training in object-oriented concepts, tools, and techniques.

3) Develop a plan to systematically rotate development personnel through tool-specific object training as required to maintain staffing levels for emerging object projects. Significant lead time must be allowed for newly trained employees to experiment with the technology before being assigned to production development teams.

4) Utilize previously trained employees to act as mentors to newly trained ones.

\section{Dependencies}

Object.A.1

Direct that Future Site Applications Should Be Object-oriented

\section{Benefits}

This is a necessary step to prepare the site to take advantage of OO technology. 


\section{Object.C.3 Establish Reward System for Code Reuse}

Responsible Organization

IRM

Recommended Initiation Date

$1 Q / 95$
Sponsoring Organization

IRM

Status

None

\section{Anticipated Duration}

12 months

\section{Resource Considerations}

Resources for this milestone are fairly low and, for the most part, can be handled within the context of current activity.

\section{Discussion}

The reward system for application developers needs to place a premium on code reuse, rather than code creation.

\section{Action Items}

1) Encourage personnel to include LADP items relating to code reuse.

2) Present code reuse awards at a section and personal level.

\section{Dependencies}

Object.P.3 Implement Online, Centrally Managed, Distributed Access for Site Object Framework Developer Documentation

\section{Benefits}

One of the primary benefits of $O O$ technology is code reuse, but one of the basic instincts of a developer is to build software yourself. Developers need to be encouraged to work within the object framework. 


\section{Object-oriented Process Milestones}

The following work flow milestones must be resolved to adopt the technology.

Object.P.1 Establish Object-oriented Documentation Requirements

Object.P.2 - Establish Object-oriented Development Methodologies

Object.P.3 Implement Online, Centrally Managed, Distributed Access for Site Object Framework Developer Documentation 


\section{Object.P.1 Establish Object-oriented Documentation Requirements}

Responsible Organization

IRM

Recommended Initiation Date

$2 \mathrm{Q} / 95$

\section{Anticipated Duration}

Six months

\section{Resource Considerations}

A team of technical specialists and technical writers contribute a total of six man-months.

\section{Discussion}

At least two different types of software documentation must be delivered for a system: the user's guide and the software maintenance document. In a system that fosters reuse, it is unnecessary to replicate Site Object Framework documentation for every application. Object software requires, instead, detailed documentation on the implementation of applicationspecific objects and documentation on how the core framework objects were utilized (as opposed to implemented). Similarly, it is less necessary to focus the user documentation on the details of how a particular Graphical User Interface (GUI) object operates (unless its behavior is different than that normally provided by that GUI object) and instead focus more on how to utilize the capabilities of the application.

\section{Action items}

1) Establish documentation team.

2) Develop standards for site framework documentation.

3) Develop standards for application specific object documentation.

4) Develop standards for object usage documentation. (Consider the possibility of an object browser as a form of object usage documentation).

5) Develop standards for application level user documentation.

6) Develop application level user documentation for the site object framework.

7) Gain IRM approval of documentation standards.

\section{Dependencies}

Object.I.1

Object.C.2

Establish Site Object Framework Team (SOFT)

Develop Site Expertise in Object-oriented Technology 


\section{Roadmap to the SRS Computing Architecture}

\section{Benefits}

Standardizing on site documentation facilitates code reuse (since other developers have a common framework for understanding documentation) and improves consistency in end-user documentation. 


\section{Object.P.2 Establish Object-oriented Development Methodologies}

Responsible Organization

IRM

Recommended Initiation Date

$1 \mathrm{Q} / 95$

\section{Sponsoring Organization}

IRM

\section{Status}

None

\section{Anticipated Duration}

12 months

\section{Resource Considerations}

A team of object specialists will require time to evaluate alternative $O O$ development methodologies and recommend one. This will require effort on the part of the specialists (12 man-months) and potentially the costs of acquiring/training on various tools which utilize these methodologies.

\section{Discussion}

Conventional analysis and development methodologies do not fit well into the object paradigm.

\section{Action ltems}

1) Form object-oriented development methodology team.

2) Evaluate object-oriented development methodologies.

3) Recommend/develop an object-oriented development methodology.

4) Gain IRM approval of object-oriented development methodology.

5) Gain site approval of object-oriented development methodology.

\section{Dependencies}

Object.I.1

Object.C.2
Establish Site Object Framework Team (SOFT)

Develop Site Expertise in Object-oriented Technology

\section{Benefits}

Several different object-oriented analysis and design methodologies exist. The site needs to choose one as the standard. 


\section{Object.P.3 Implement Online, Centrally Managed, Distributed Access for Site Object Framework Developer Documentation}

Responsible Organization

SOFT/IRM

Recommended Initiation Date

$3 Q / 95$
Sponsoring Organization

IRM

Status

None

\section{Anticipated Duration}

Six months

\section{Resource Considerations}

Most of the resources necessary to support this effort exist within the WISDOM framework. It is also highly desirable that developer documentation templates be derivable from the object source code. Tools to perform this service are freely available for many object environments, but undoubtedly, will have to be modified to conform to the site standard. The amount of effort required is dependent upon the selected object tool standard, but a pessimistic estimate is six man-months. A modest effort on the part of the SOFT will be required to act as the librarian for this documentation.

\section{Discussion}

The primary vehicle for code reuse will be the Site Object Framework. To maximize the reuse of this framework, the developer documentation must be consistently updated to reflect the latest extensions and modifications. Electronic access to a central document database is the only viable approach to making this information available in a timely fashion.

\section{Action Hems}

1) Establish a team composed of networks, WISDOM, development, and object technologists to specify a tool that provides documentation templating based upon class definitions, per the site documentation standard.

2) Develops the tool.

3) Provides a common access method for resultant documentation (that is, a documentation database).

\section{Dependencies}

Object.P.1

Establish Object-oriented Documentation Requirements

\section{Benefits}

Ready access to current documentation (online) will not only result in more correct use of the current revision level of the SOF, but will increase the likelihood of timely updates to that documentation as well. 


\section{Object-oriented Technology Milestones}

Object.T.1

Object.T.2

Object.T.3

Object.T.4

Object.T.5
Survey Industry Experience in Migrating Legacy Systems to an Object-oriented Environment

Demonstrate Relational Database Access from Object-oriented Systems

Evaluation of Operating Systems (Object-oriented)

Evaluation of Object-oriented Development Tools

Explore Distributed Object Server Technology 


\section{Object.T.1 Survey Industry Experience in Migrating Legacy Systems to an Object-oriented Environment}

Responsible Organization

IRM

Recommended Initiation Date

$2 Q / 94$
Sponsoring Organization

IRM

Status

Underway

\section{Anticipated Duration}

36 months

\section{Resource Considerations}

The lengthy duration of this milestone is not a reflection of the amount of effort it requires, but more a recognition that this needs to be an on-going effort during WSRC's object transition. An effort of one man-month per year is probably sufficient.

\section{Discussion}

WSRC is not the only organization that is faced with migration to an object environment. Other companies are pursuing this path as well and the site is well, advised to take advantage of as much experience from other organizations as possible.

\section{Action ltems}

1) Pursue joint projects with other organizations within DOE.

2) Close association with end-user object action groups.

3) Form a continue literature research.

\section{Dependencies}

None

\section{Benefits}

Studying the successes and failures of other companies in their object migration can save SRS from making the same mistakes and provide guidance on how to make the transition in a costeffective manner. Joint projects with other sites may also lead to additional extensions to the Site Object Framework. 


\section{Object.T.2 Demonstrate Relational Database Access from Object-oriented Systems}

Responsible Organization

IRM

Recommended Initiation Date

$4 \mathrm{Q} / 94$

Anticipated Duration

12 months

Resource Considerations

Discussion

During a long transitional period, it is important that object-oriented applications coexist with traditional applications and that data can be shared between legacy applications and object applications.

Action ltems

1) Test object-oriented database access to relational databases.

2) Test Oracle access via object-oriented development tool.

Dependencies

None

Benefits 


\section{Object.T.3 Evaluation of Operating Systems (Object-oriented)}

Responsible Organization

IRM

Recommended Initiation Date

$1 \mathrm{Q} / 95$

\section{Anticipated Duration}

12 months

\section{Resource Considerations}

Initially, this will require small output of resources in order to keep informed of what possibilities exist within the commercial world (two to three man-months per year). As the tools reach maturity, the site may elect to expend more effort on a full-scale evaluation of one or more potential operating systems.

\section{Discussion}

To determine the site standard for object-oriented operating systems, we must explore the available options. Note the tenuous nature of the recommended starting date for this milestone. As yet, $O O$ operating systems are virtually nonexistent (PenPoint excepted). However, several key vendors are poised to supply these tools (that is, Cairo, Taligent, NextStep). The start date of this milestone is dependent upon vendor readiness. 1Q/95 is expected to be the earliest time when the site will be able to begin working with this technology.

\section{Action Hems}

1) Obtain sample versions of announced object-oriented operating systems as available.

2) Develop close working relationships with major vendors.

\section{Dependencies}

None

\section{Benefits}

OO operating systems provide the benefits of object orientation at the operating system and utilities level. These systems will also remove a boundary at which $O O$ applications are forced to act in procedural ways (to interact with non-OO operating systems). 


\section{Object.T.4 Evaluation of Object-oriented Development Tools}

\section{Responsible Organization}

IRM

Recommended Initiation Date

$1 \mathrm{Q} / 94$
Sponsoring Organization

IRM

\section{Status}

Under way

\section{Anticipated Duration}

36 months

\section{Resource Considerations}

This milestone will require more resources than might be suspected at first. Learning to fully use a development tool is not a quick proposition. Time is required to fully understand the possibilities and the shortcomings of a particular development tool. A minimum of three manmonths per tool should be allowed. At present, there are half a dozen serious contenders for a site standard object development tool.

\section{Discussion}

To determine the site standard for object-oriented development tool systems, we must explore the available options. Several tools exist for this purpose, each with its own strengths and weaknesses. Careful examination of the alternatives should be made prior to selecting a site standard.

\section{Action Items}

1) Obtain object-oriented development tools as available.

2) Develop close working relationships with major vendors.

\section{Dependencies}

None

\section{Benefits}

One of the most powerful (and time-saving) features of most object development environments is the strength of the development tools that are offered. To a large degree, selection of a good tool will allow the site to obtain the full benefit of objects. Conversely, selection of a poor tool will restrict the amount of benefit that objects can provide. 


\section{Object.T.5 Explore Distributed Object Server Technology}

Responsible Organization

IRM

Recommended Initiation Date

$4 \mathrm{Q} / 94$
Sponsoring Organization

IRM

Status

None

\section{Anticipated Duration}

18 months

\section{Resource Considerations}

As should be obvious from the action items, this is a fairly low-level effort (two man-months) until the pilot phase. The pilot may require as much as six man-months.

\section{Discussion}

One of the major unanswered questions about object-oriented technology is the mechanism for sharing global site objects between applications. While some of the Object Management Group's (OMG) effort is addressing this issue, a solution is far from clear. WSRC will have to monitor developments of this key technological component carefully.

Action ltems

1) Continue literature research on distributed objects.

2) Obtain associate membership in CORBA.

3) Obtain participatory membership in object user organizations.

4) Conduct pilot studies of emerging distributed object technologies.

\section{Dependencies}

None

\section{Benefits}

Allows the site to prepare to take advantage of emerging distributed object messaging standards. 


\section{Bibliography}

1. WSRC-IM-91-18-1, Savannah River Site Computing Architecture

2. WSRC-IM-90-83-1, Reviews of Computing Technology: Client/server Technology

3. WSRC-IM-90-83-6, Reviews of Computing Technology: Fiber Distributed Data Interface

4. WSRC-IM-92-23, SRS Computing Standards and Guidelines Reference

5. WSRC-IM-92-109, SRS Application Design Architecture

6. WSRC-93-14-2, SRS Computing Infrastructure Design Architecture

7. WSRC, OVP-930155, Financial and Business Computer Systems Action Plan 


\section{Appendix A: Glossary}

10baset

Ethernet local area network utilizing unshielded twisted pair (UTP) cables

3270

A low-speed character-cell terminal interface to IBM Mainframe systems.

$4 G L$

Fourth-generation language. A type of computer language that accepts system requirements as input and generates computer code to meet those requirements as output.

\section{ADABAS}

Database management system that supports most legacy applications on the IBM Central Computing Facility. ADABAS is non-relational.

ADP

Automated Data Processing.

AIX

The version of UNIX that runs on the IBM RS/ 6000 .

ALD

Administration and Logistics Division of WSRC.

ANSI

American National Standards Institute: a national standards body.

Analysis

Separation of an intellectual or substantial whole into its constituent parts for individual study. The stated findings of such separation or determination.

API

Application program interface. Protocols and software that enable consistent interfaces for communications and transfer of information between applications.

Application

The data manipulation and processing operations that are related to specific requirements of an information system. Software or program that is specific to the solution of a specific business problem or process. 


\section{Application System}

A collection of applications which utilizes the services provided by the human-computer interface, communications facility, and data management system to perform the processing necessary to meet the requirements of the information system.

\section{Architecture.}

The manner in which hardware, software, or data is structured. Architecture typically describes how the system or program is constructed, how its components fit together, and the protocols and interfaces used for communication and cooperation among modules or components of the system.

\section{Archive}

To place infrequently accessed data into permanent storage.

\section{AT Bus}

The cardset form-factor for original IBM PCs and the mass installed base of so-called "clones." Still a dominant bus in consumer and business computers. Also called the ISA bus.

\section{ATM}

Adobe Type Manager: a packaged set of Postscript fonts and associated utilities.

Asynchronous transfer mode. A new network strategy that provides dedicated 100 megabit per second network connections between workstations and servers for the duration of the session.

\section{Attribute}

A single fact about a business entity or relationship. A property inherent in, or associated with, an entity. (Referred to as a data element in an information system.)

\section{BOOTP}

Standard for providing IP addresses to workstations dynamically.

\section{BSRI}

Bechtel Savannah River Incorporated.

\section{Business Rules}

The basic operation constraints under which a given organization does business. A statement reflecting a policy, operating standard of practice, or some other form of constraint which governs the correctness and usefulness of enterprise information. A business rule statement must always be true within the context to which it applies, never sometimes true or partially true

C/S

Client/Server, a style of computing based on cooperating computers, especially utilizing an intelligent workstation.

\section{CASE}

Computer Aided Software Engineering. 


\section{CASE TOols}

A wide range of software products that provide automated productivity and quality support, to varying degrees of rigor, for phases (stages) of applications development and maintenance. For example, CASE tools cán allow application developers to perform analysis and design using a commonly understood graphical representation, such as Yourdon, Gane \& Sarson, Jackson, or Chin's diagrams. In the database arena, CASE tools are used to build and test database designs on the workstation and to port these designs to a host computer.

\section{CAST}

Computing Architecture and Standards Team.

CCF

Central Computer Facility.

CDA

Compound Document Architecture, a strategy for combining text, graphics, and other datatypes.

\section{CD or CD-AOM}

Compact disc. A 5 inch disk containing digital information introduced by Sony and Phillips in 1982. It holds 660 megabytes of information that is read by laser beam.

\section{Change Control}

The process of managing changes to individual components within each version of information that may include source code, version control, documentation, and system configuration.

The process of controlling, authorizing, and tracking changes to manual or automated data.

\section{Class (Class Object)}

In object-oriented technology a template for the creation of a group of instances that share common characteristics. A class is also an object in its own right and, as such, has access to class data and methods and can respond to messages.

Client

The workstation and/or software running at the workstation in a distributed computing environment.

\section{ClientServer}

Cooperative processing is a general computing model in which an application's functions are logically split such that two or more complementary programs interact and execute concurrently on one (typically, two or more) or more machines as part of an overall business function. Client/Server is a form of cooperative processing in which one of the participating applications is a personnel work station through which the user interacts with the network. To qualify as Client/Server, the workstation must execute some of the application logic beyond emulation:

Client: The driving or initiating component on an workstation, delegating redefined types of tasks to a server and making requests of a server, usually for which it awaits a response. 
Server: A host acting on behalf of a client for predefined functions, for example, database requests.

Clone

The generic term for computer that utilizes the Intel microprocessor typically running DOS and/or Windows applications.

\section{Computer-Aided Software Engineering (CASE)}

The use of automated interactive graphical design techniques, design checking rules, encyclopedias of design elements, and simulation to drive or facilitate the analysis, design, documentation, construction, and maintenance of computer applications.

\section{Computer System}

A collection of hardware which is managed as a single unit by software such as an operating system which may also provide common services such as access control, interprocess communications, and a Graphical User Interface.

\section{Configuration Management}

The environment that establishes and administers the process for uniform change control to ensure stability, consistency, reliability, quality, and traceability of any/all forms of change within any enterprise.

The systematic evaluation, coordination, and approval or disapproval of changes, and the implementation of all approved changes of an item after formal establishment of its configuration identification.

\section{CORBA}

Common Object Request Broker Architecture. Emerging standard from the Object Management Group (OMG) for messaging between objects from multiple vendors.

\section{DASD}

Direct Access Storage Device, a magnetic disk storage system for IBM mainframes.

\section{Data}

A representation of facts, concepts, or instructions in a formalized manner suitable for communication, interpretation, or processing by humans or by automatic means.

Symbols, written or stored on some recording medium, representing certain things, ideas, or values and conveying meaningful information in particular contexts; the encoded representation of information.

\section{Data Administration}

The function of developing and administering the policies, procedures, practices, and plans for the definition, organization, protection, and efficient use of data as a corporate resource.

\section{Database}

A collection of data logically organized to meet the information and time requirements of a universe of users. 
A collection of interrelated, largely unique, data items or records, in one or more computer files, that may be processed by many different application programs.

\section{Database Administrator (DBA)}

An individual with responsibility for one or more databases, who controls the design and use of these databases.

\section{Data Collection}

A collection of data implemented as a unit, which contains facts of interest to the enterprise.

\section{Data Dictionary}

A software package that manages the storage of information about data such as data item names, definitions, formats, and relationships to other data.

A description of the characteristics of data. The system tables that contain descriptions of the database objects and how they are structured.

An inventory that describes, defines, and lists all of the data elements that are stored in a database.

\section{Data-Driven}

A development philosophy that emphasizes data architecture design separate and independent of application functionality.

A term used to describe the orientation of an information engineering approach to information resource management. This approach results in optimum reliability, sharability and usefulness of information across all enterprise activities. It maximizes the reusability of all system components.

\section{Data Integrity}

The ability to preserve the completeness, currency, and accuracy of the data without unintentional changes; the ability to produce results that are correct to a predefined level and to maintain data availability.

\section{Data Management}

A general term that collectively describes those functions of the system that provide creation of, and access to, stored data, enforce data storage conventions, and regulate the use of input/output devices.

\section{Data Stewardship Program}

A concept that defines individual and company responsibility to manage corporate data resources in compliance with established standards, policies, and procedures. The Program provides a consistent approach for effective data management across the entire company.

\section{DC\&S}

Digital Control \& Systems (Department) of WSRC

DB2

IBM's primary relational database management system. 
DBA

Database Administrator.

\section{DBMS}

Database Management System.

DCE

Distributed Computing Environment (OSF).

DEC

Digital Equipment Corporation.

\section{Derived Data}

Data values that are derived from the values of other data by a specified algorithm.

DMS

Document Management System: a major WSRC procurement to improve document and records automation planned for 1995.

DOE

Department of Energy

Domain

The general area of expertise of a particular expert system.

DOS

Disk Operating System developed by Microsoft for the IBM-PC. (also MS-DOS , PC-DOS)

E-mail

Electronic mail, a computer-based messaging system.

EDI

Electronic Data Interchange: a standard format and protocol for the exchange of business information (typically numeric and database fields).

Encapsulation

A term describing the indivisible nature of the data and operations that make up an object.

Enterprise

A government agency; a contractor with a government agency; an organization in the public sector; or a company in the private sector.

Used in place of such terms as corporation, company, government agency, government program and firm. Generally applies to any kind of organization with autonomous control over its own approach to information resource management. 
Ethernet

Local area network technology (DEC/XEROX/INTEL).

FAX

A scanned image of a paper document transmitted over telephone lines.

FDDI (Fiber Distributed Data Interface)

A token-passing LAN that uses optical cabling to pass data at speed up to 100 megabits per second. Recent implementations can use site universal wiring.

File

A collection of related data that is stored and retrieved by an assigned name; synonymous with data set.

A collection of rows (or records) that have associated columns (or files); the logical equivalent of a table.

A named set of records stored or processed as a unit.

FTE

Full-time equivalent: the cost or effort expended by an employee in a 12 month period.

\section{GB (Gigabyte)}

A measure of data storage approximately equal to 1 billion characters.

GUI

Graphical User Interface.

HTML

Hypertext markup language. A standard set of tags used in preparing a document for hypertext retrieval.

Hub

Device used to build 10BaseT local area networks.

\section{IBM}

International Business Machines Corporation

Information Resource Management

The study, planning, management, and control of information as a corporate asset. IRM encompasses the management of network, hardware, data, software, and organization resources. .

\section{IP Address}

Internet Protocol address, a unique numeric assignment to a host or workstation used for network connection in an Internet data network. 
IRM

Information resource management function or Information Resource Management (Department) of WSRC

IS

Information Systems.

IT or IT

Information Technology.

IW

Information Warehouse.

KB (Kilobyte)

A measure of data storage approximately equal to 1,000 characters.

\section{Kerberos}

A network login and authentication standard that allows a single login to access multiple network resources.

\section{LAN}

Local Area Network.

Legacy Data (System)

Existing data or applications to which integration or migration must occur.

Local Area Network (LAN)

The interconnection of computers (PCs, workstations, mid-range, and mainframes) in a building or campus to enable the sharing of files, printers, and other resources.

Macintosh

Apple Computer's personal computer family.

Mainframe

Large computer system (often associated with IBM), usually associated with running an organization's major business systems

\section{MB (Megabyte)}

A measure of data storage approximately equal to 1 million characters.

\section{Mbps (Megabits per second)}

LAN transfer rate expressed in terms of bits rather than bytes. A byte is nominally 8 bits, so a $10 \mathrm{Mbps}$ LAN has the potential for data transfer at the rate of $1.25 \mathrm{MB}$ per second.

\section{Methodology}

A structured, procedural process that decomposes system development into phases with phasespecific tasks and well-defined deliverables. 
MIB

Management Information Base, a collection of information describing how devices can be managed in a NMS using SNMP.

\section{Middleware}

Software services that reside above a system's basic operating system that provide data access and communications services for application software.

MIE

Major Item of Equipment: a procurement exceeding $\$ 1,000,000$.

MSD

Management Services Department of WSRC.

NARA

National Archives and Records Administration: the government affiliated standards body for records management.

Network

A computer communications system linking a series of computer elements. A system of interconnected computing devices that can communicate and share resources. Networks may be private (for one user) or shared (for many users).

\section{NeXTStep}

Object Development/Execution environment developed by NeXT Computer, Inc. Generally regarded as the most complete object environment commercially available today.

NMS

-Network Management System, a comprehensive system for managing a data network.

NOS

Network Operating System, software that provides file and printer services across a LAN.

Novell

Novell Inc., vendor of Netware, the site standard NOS.

Object

A software packet containing a collection of related data (in the form of variables) and methods (procedures) for operating on that data. It is a specific instance of a class and includes the characteristics of that class.

Object Framework

A hierarchical collection of object classes defining a set data and behaviors (operations) and the relationship between these object classes. Objects within the framework pertain to a central concept (that is, User Interface or Human Resources). Frameworks can themselves be combined to create larger frameworks. 
Object Management Group (OMG)

Industry group dedicated to promoting object-oriented technology and establishing OOT standards.

OCR

Optical Character Recognition: conversion of image pages back to the text that comprises the printed words.

ODA

Open Document Architecture: an international standard for compound document representation.

00

Object-oriented

OOP

Object-oriented programming

OOPS

Object-oriented Programming System.

OSF

Open Systems Foundation, an industry consortium that promotes open computing standards

PC

Personal Computer (for example, Macintosh, IBM PS/2).

PDF

Portable Document Format: Adobe's Postscript-based format for cross-platform view/print document interchange products.

PMMD

Procurement \& Materials Management Department of WSRC

Quicktime

Apple Macintosh digital video architecture.

RAM

Random Access Memory: the volatile storage in a computer system.

RDBMS

Relational Database Management System.

Reference Data

Includes all the common data elements for which a business establishes and imposes a consistent structure and a limited set of values. Reference data has meaning and definition 
across WSRC and is used for reference and validation. Reference data is fundamental to the operation of the business.

\section{Referential Integrity}

That property of a relational database management system that enables it to prevent the introduction of inconsistent data into the database. For instance, when a new employee is entered into the database, he or she cannot be assigned to a department that does not exist. RI consists of relationships, or rules, that a database designer defines. These rules describe pairs of tables in which the values of the rows in one table depend on the value of the rows in another table (as in the above example).

\section{Relational Database}

A database that is organized and accessed according to relationships between data items.

A data structure perceived by its users as a collection of tables. A relational database consists of tables, rows, and columns. Most mini-computers and frames today have relational database systems available for business use. Typical examples are DB2 from IBM and RDB from Digital Equipment Corp. Relational databases differ from non relational databases in that there are no system dependencies stored within the data; for example, hierarchical databases are not relational because they contain pointers to other data. SQL Server is a relational DBMS.

A data base management system in which data is represented in two-dimensional tables. A DBMS with the integrity, structural, and manipulative features of the relational model.

\section{Repository}

A database that contains metadata, that is, information about the data itself (where data is located, what names are used, format, rules, etc.) and other application components.

\section{Router}

Data communications device for joining networks together.

APC

Remote Procedure Call.

RTS

Replacement Telephone System, new telephone system for SRS.

SDM

System Development Methodology: a set of guidelines for software development and software project management.

\section{SEH}

Switched Ethernet Hub, a hub that provides a dedicated Ethernet bandwidth to each attached workstation.

\section{Server}

A computer system that provides one or more specific support functions for workstations such as file sharing, applications storage, or databases. 
SGML

Standard Generalized Markup Language: an international set of standards for tagging compound documents.

SNMP

Simple Network Management Protocol, an open protocol instrumental in developing a NMS

SOFT

Software Object Framework Team. A proposed WSRC organization with primary responsibility for the development and maintenance of the site object framework.

Software

Computer code that can be compiled.

All or part of the programs, procedures, rules, and associated documentation of computer system.

SQL

Structured Query Language.

SRS

The Savannah River Site, a Department of Energy facility in Aiken, South Carolina.

SRSnet

The SRS data communications network

Strategic Information/Data Planning

The development of plans to support the organization's long-range information, data, and applications systems requirements.

Defining data requirements from strategic business plans, developing global logical data models, establishing data policies, assessing opportunities, and relating information planning strategies to business requirements.

System

An organized combination of the services of machines, materials, and people to accomplish a function.

A collection of data processing jobs and application programs that interact to perform a business function (that is, ledger system, financial data system, environmental control and tracking system).

\section{System Devẹlopment Life Cycle (SDLC)}

The time span and the spheres of activity from the inception of a system until it is abandoned or replaced. This time span generically includes system analysis, design, development/coding, implementation, operation, and maintenance. 


\section{Roadmap to the SRS Computing Architecture}

T1

Digital telephone circuit rated at 1.544 million bits per second.

Taligent

Object-oriented envirōnment under development by Taligent, Inc. (a joint venture of IBM and Apple). Slated for beta release in early 1995, Taligent promises to deliver a robust, operating system independent enviroment for object execution.

TCPIIP

Transmission Control Protocol / Internet Protocol, the set of open communications protocols that were developed to build the Internet global computer network.

$\mathrm{TI}$

Training Integration (Department) of WSRC.

TP\&C

Technical and Process Computing (Department) of WSRC

Trigger

Also called "database event alerters," triggers allow the database server to communicate information about database events to a client application. In turn, triggers can be configured to fire off one or more business rules, run an SQL script against the database, run a program on the client or server computer, or simply alert a user that an event has occurred.

UNIX

Popular computer operating system, invented aty AT\&T, and ported to many different types of computer systems.

\section{Universal Wiring}

Site standard for installing UTP for data networks

UTP

Unshielded Twisted Pair, data grade telephone wiring based on 4 pairs of copper wire.

User

Anyone who uses data.

Anyone who uses the services of a computer system or its products.

VAX

Primary DEC computer family.

VCR

Video cassette recorder.

VMS

Virtual Memory System (VAX operating system). 


\section{WAN}

Wide Area Network, a data network based on leased telephone circuits and connecting geographically dispersed locations.

\section{Windows (Microsoft Windows)}

The industry standard windowed operating system for Intel 386 and 486 computers running DOS.

\section{WISDOM (Workstation Integration System for Desktop Office Machines)}

The WSRC implementation of Windows and Macintosh workstations in a networked configuration.

\section{Workstation}

A computer designed to support a single user for applications such as word processing, spreadsheets, databases, and calculational programs to accomplish work. Workstations are often categorized as either personal computers or scientific/engineering workstations based on performance levels and the tasks which they are designed to accomplish.

\section{WSRC}

Westinghouse Savannah River Company, the prime contractor for the Department of Energy's Savannah River Site in South Carolina.

\section{$X .500$}

International standard for electronic directories. 


\section{Appendix B: Roadmaps Milestones Summary}

\section{Milestone}

Networks.I.1 Install the Replacement Telephone System

Networks.I.2 Complete Intra-area Cabling

Networks.I.3 Complete Site Universal Wiring

Networks.I.4 Complete Building LANs

Networks.I.5 Install Ethernet/FDDI Network Trunks

Networks.I.6 Install Switched Ethernet Hubs

Networks.I.7 Install ATM Network Trunks

Networks.I.8 Install ATM Workgroup Networks

Networks.I.9 Complete Network Management System

Networks.I.10 Initiate Network Authentication Service

Networks.I.11 Install Wireless LANs as Extension to SRSnet

Networks.I.12 Complete Analysis of Network, Telephone, and

Dedicated Fiber Usage Requirements

Networks.I.13 Provide Access Between SRSnet and Offsite Locations

Networks.A.1 Set Site Direction, Funding, and Priority

Networks.A.2 Relocate Most Network Users Onsite

Networks.A.3 Resolve Security and Spectrum Management Issues for Wireless LAN

Networks.A.4 Obtain Computer Security Endorsement of Networkbased Authentication
Start Date Duration

$\begin{array}{ll}\text { Jan-94 } & 24 \\ \text { Jan-94 } & 24 \\ \text { Jan-94 } & 24 \\ \text { Jan-94 } & 24 \\ \text { Jan-94 } & 36 \\ \text { Oct-94 } & 36 \\ \text { Jan-96 } & 48 \\ \text { Oct-95 } & 48 \\ \text { Jan-94 } & 36 \\ \text { Jan-95 } & 36\end{array}$

Oct-94

24

Oct-94

24

Jul-94

18

Jan-94

Jan-94

60

Apr-94

24

Apr-94 24 
Networks.A.5 Resolve Security and Network Use Issues for Offsite Access to and from SRSnet

Networks.T.1 Implement Cost-effective Switched Ethernet Hubs

Jan-94 24

Networks.T.2 Compiete ATM Standards

Jan-94 36

Networks.T.3 Complete ATM Interfaces for Routers/Hubs

Networks.T.4 Complete ATM Interfaces for Workstations/Host

Oct-94

Computers

Networks.T.5 Make DCE Security Services Available in Key

Jan-95

Environments

Netsys.I.1 Install Robust Client Workstations

Jan-94

24

Netsys.I.2 Upgrade Workstations to Support Netsys Requirements

Apr-94

36

Netsys.I.3 Ensure Broad Availability of Networked Workstations

Apr-94

24

Netsys.I.4 Migrate to New NOS

Apr-94

Netsys.I.5 Initiate NOS Global Directory

Apr-94

12

Netsys.I.6 Initiate Network Authentication Service

Jan-95

Netsys.I.7 Complete Installation of Territorial File Servers

Jan-94

30

Netsys.I.8 Manage IP Address

Jan-94

6

Netsys.I.9 Implement Robust File/Print/Application Services

Jul-94

24

Netsys.I.10 Implement Automatic Software Distribution

Jan-94

36

Netsys.I.11 Connect Office Peripherals Directly to Network

Jan-94

Netsys.I.12 Implement Reliable Performance Monitoring

Jul-94

Netsys.A.1 Set Site Direction, Funding, and Priority

Jan-94

Netsys.A.2 Define Security Principles

Apr-94 
Netsys.A.3 Establish NOS Operations Group

Apr-94

12

Netsys.C.1 Implement Standards

Apr-94

24

Netsys.C.2 Share Equipment/Peripherals

Jan-94 36

Netsys.C.3 Save Data to Network

Jan-94 36

Netsys.P.1 Implement Single User Login to Network

Jul-94

24

Netsys.P.2 Implement Distributed Workgroup Management

Jul-94

36

Netsys.P.3 Implement Software License Management

Oct-94 $\quad 30$

Netsys.P.4 Implement Disaster Recovery Procedure

Jul-94

12

Netsys.P.5 Implement Infrastructure Enhancement Process

Apr-94

24

Netsys.T.1 Deliver NOS over TCP/IP

Jan-95

12

Netsys.T.2 Implement Transparent Print Services on All Platforms

Oct-94

12

Netsys.M.1 Implement PC Utilization Plan

Apr-94

12

IW.I.1 Establish a Communications Network Capability Supporting the Global and Local Warehouses

Jan-94

48

IW.I.2 Establish Client SQL-Network

Apr-94

24

IW.I.3 Establish/Populate IW Component of Data Repository

Jan-94

36

IW.I.4 Identify and Procure IW Support Tools and Hardware

Jan-94

18

IW.I.5 Identify and Procure Archival Management Tools and Hardware

Jan-95

12

IW.A.1 Establish IW Implementation Team

Jan-94

6

IW.A.2 Establish Long-term Support Organization

Jul-94

3

IW.A.3 Establish IW Polices and Procedures

Apr-94

6 
IW.A.4 Replace Redundant Operational Reports, Screens, and Data

Feeds

IW.C.1 Establish Training for Applications-development Community

Apr-94

IW.C.2 Establish Training for End-User Community

Oct-94

24

IW.P.1 Identify and Prioritize Business Requirements for Global and

Jan-94

6

Local Warehouses

IW.P.2 Define the IW Model

Apr-94

9

IW.P.3 Implement the Global Warehouse

Jul-94

24

IW.P.4 Expand Global Warehouse

Jul-96

24

IW.P.5 Establish Local or Remote IWs

Jan-95

18

IW.P.6 Establish Archival IW

Jul-95

12

C/S.I.1 Install Robust Development Workstations

Jan-94

12

C/S.I.2 Implement Locally Distributed, Centrally Managed RDBMS

$\operatorname{Jan}-94$

24

Servers

C/S.I.3 Implement Single Network Transport Protocol for

Jul-94

3

Client/Server Communication

C/S.A.1 Develop Graphical User Interface Guidelines

Apr-94

6

C/S.A.2 Develop Naming Conventions

Apr-94

6

C/S.A.3 Enable Sitewide Data Access

Oct-94

12

C/S.A.4 Encourage Move Toward Client/Server Development

Jan-94

C/S.A.5 Migrate Selected Host-Based Legacy Systems to Client/Server

Apr -94

48

C/S.C.1 Establish Small Development Team Methodology

Jan-94

24

C/S.C.2 Establish Policies Favoring Iterative Software Development

Apr-94

24 
C/S.P.1 Implement Standard Development Methodology and Tools Jan-94 Across Site

C/S.P.2 Integrate Software Tools, Development Methodology, Project, Jan-94 and Configuration Mānagement Products

C/S.T.1 Implement Standard RDBMS Accessibility

Apr-94

C/S.T.2 Implement Graphical Report Generators and Query Tools

Jul-94

C/S.T.3 Implement Nonrelational Database Access Through SQL Gateway

Apr-94

6

C/S.T.4 Implement Declarative Referential Integrity, Triggers, and Stored Procedures Supported by RDBMS

C/S.T.5 Implement Monitoring Tools

Apr-94

C/S.T.6 Implement Cross-platform Two-phase Commit Regardless of Vendor

Oct-95

MMedia.I.1 Develop Multimedia Principles

Jan-94

MMedia.I.2 Adopt Cross-platform Fonts and Image Formats

$\operatorname{Jan}-94$

MMedia.I.3 Establish Navigable Compound Document Format

Apr-94

MMedia.I.4 Enable Routine Capture and Playback of Cross-platform

Apr-94

Audio

MMedia.I.5 Implement Storage, Multimedia Servers, CD-ROM, and Improved Monitors

MMedia.I.6 Enable Playback Motion Video at Desktop

Jan-95

MMedia.I.7 Implement Workgroup Video-capture Stations

Jul-95

MMedia.I.8 Implement Cross-platform Authoring Tools

Jul-95

MMedia.A.1 Approve Use of Multimedia Data Types

Apr-94

MMedia.A.2 Formulate Policy for Authentication of Nonprintable

Jul-94 
MMedia.A.3 Determine Business Case for Desktop Video Capture

MMedia.P.1 Manage Stored Digital Video Clips

Jul-95

MMedia.P.2 Implement Routine Use of Multimedia Data in Office

Jan-95 24

Documents

MMedia.T.1 Pilot Multimedia Enablement of Desktop Products

$$
\text { Jan-94 }
$$

MMedia.T.2 Pilot Navigable Compound Documents

$$
\text { Apr-94 }
$$

MMedia.T.3 Pilot Speech and Handwriting Recognition

$$
\text { Apr-95 }
$$

MMedia.T.4 Pilot Applicability of Object Databases to Multimedia

$$
\text { Jul-95 }
$$

MMedia.T.5 Pilot Desktop Videoconferencing

$$
\text { Jan-95 }
$$

Office.I.1 Implement FAX Input/Output

$$
\text { Jan-94 }
$$

Office.I.2 Install Online Scanners

$$
\text { Jan-94 }
$$

Office.I.3 Install Ubiquitous OCR

$$
\text { Jan-94 }
$$

Office.I.4 Install Client/Server E-mail

$\operatorname{Jan}-94 \quad 36$

Office.I.5 Implement Mail-enabled Applications

Oct-94 $\quad 30$

Office.I.6 Implement Electronic Forms Routing and Authorization

Jul-94 30

Office.I.7 Implement Viewers/Translators

Office.I.8 Integrate Voice-mail and E-mail Systems Jan-94 36

Office.A.1 Implement Security Policy and Procedures

Office.A.2 Scan in Historical and Reference Data 
Office.A.6 Implement the Paperless Office

Office.P.1 Implement Document Management System

JuI-94

24

Office.P.2 Implement Electronic Data Interchange

Jul-96

18

Office.T.1 Implement Voice Processing

Jan-97

Object.I.1 Establish Site Object Framework Team (SOFT)

Jul-94

Object.I.2 Adopt Site Object Messaging Standard

Jul-94

Object.I.3 Establish Object Framework

$\operatorname{Jan}-95$

Object.I.4 Determine and Implement the Necessary Changes to

Oct-94

6

WISDOM to Support an Object-oriented Environment

Object.I.5 Re-engineer Existing Core as Object Applications

Oct-96

Object.A.1 Direct that Future Site Applications Should be Object-

Jul-94 oriented

Object.A.2 Procure Object-oriented Technology

Object.A.3 Develop Object Migration Plans for Existing Systems

Object.C.1 Implement Matrix Project Team Approach

Object.C.2 Develop Site Expertise In Object-oriented Technology

Jan-95

Object.C.3 Establish Reward System for Code Reuse

Jan-95

Object.P.1 Establish Object-oriented Documentation Requirements

Object.P.2 Establish Object-oriented Development Methodologies

Object.P.3 Implement Online, Centrally Managed, Distributed Access

Object.T.1 Survey Industry Experience in Migrating Legacy Systems to an Object-oriented Environment 
Object.T.2 Demonstrate ReN' ional Database Access from Objectoriented Systems

Object.T.3 Evaluation of Operating Systems (Object-oriented)

Jan-95

12

Object.T.4 Evaluation of Object-oriented Development Tools

Jan-94

36

Object.T.5 Explore Distributed Object Server Technology

Oct-94

18 